\title{
Metric and Topological Spaces
}

\author{
T. W. Körner
}

December 6, 2021

Small print The syllabus for the course is defined by the Faculty Board Schedules (which are minimal for lecturing and maximal for examining). What is presented here contains some results which it would not, in my opinion, be fair to set as book-work although they could well appear as problems. In addition, I have included a small amount of material which appears in other $1 \mathrm{~B}$ courses. I should very much appreciate being told of any corrections or possible improvements and might even part with a small reward to the first finder of particular errors. These notes are written in $\mathrm{LT}_{\mathrm{EX}} 2_{\varepsilon}$ and should be available in tex, ps, pdf and dvi format from my home page

$$
\text { http://www.dpmms.cam.ac.uk/ twk/ }
$$

I can send some notes on the exercises in Sections 15 and 16 to supervisors by e-mail.

\section{Contents}

1 Preface 2

2 What is a metric? $\quad 3$

$\begin{array}{lll}3 & \text { Continuity and open sets for metric spaces } & 7\end{array}$

4 Closed sets for metric spaces 10

5 Topological spaces $\quad 12$

6 Interior and closure $\quad 14$

$\begin{array}{lll}7 & \text { More on topological structures } & 16\end{array}$

8 Hausdorff spaces $\quad 20$

9 Compactness $\quad 22$

10 Products of compact spaces $\quad 26$ 
12 Compactness in metric spaces $\quad 31$

13 The language of neighbourhoods 33

14 Final remarks and books $\quad 36$

$\begin{array}{ll}15 \text { Exercises } & 37\end{array}$

16 More exercises $\quad 45$

17 Some hints $\quad 55$

$\begin{array}{ll}18 \text { Some proofs } & 57\end{array}$

$\begin{array}{ll}19 \text { Executive summary } & 91\end{array}$

\section{Preface}

Within the last sixty years, the material in this course has been taught at Cambridge in the fourth (postgraduate), third, second and first years or left to students to pick up for themselves. Under present arrangements, students may take the course either at the end of their first year (before they have met metric spaces in analysis) or at the end of their second year (after they have met metric spaces).

Because of this, the first third of the course presents a rapid overview of metric spaces (either as revision or a first glimpse) to set the scene for the main topic of topological spaces.

The first part of these notes states and discusses the main results of the course. Usually, each statement is followed by directions to a proof in the final part of these notes. Whilst I do not expect the reader to find all the proofs by herself, I do ask that she tries to give a proof herself before looking one up. Some of the more difficult theorems have been provided with hints as well as proofs.

In my opinion, the two sections on compactness are the deepest part of the course and the reader who has mastered the proofs of the results therein is well on the way to mastering the whole course.

May I repeat that, as I said in the small print, I welcome corrections and comments. 


\section{What is a metric?}

If I wish to travel from Cambridge to Edinburgh, then I may be interested in one or more of the following numbers.

(1) The distance, in kilometres, from Cambridge to Edinburgh 'as the crow flies'.

(2) The distance, in kilometres, from Cambridge to Edinburgh by road.

(3) The time, in minutes, of the shortest journey from Cambridge to Edinburgh by rail.

(4) The cost, in pounds, of the cheapest journey from Cambridge to Edinburgh by rail.

Each of these numbers is of interest to someone and none of them is easily obtained from another. However, they do have certain properties in common which we try to isolate in the following definition.

Definition 2.1. Let $X$ be a set ${ }^{1}$ and $d: X^{2} \rightarrow \mathbb{R}$ a function with the following properties:-

(i) $d(x, y) \geq 0$ for all $x, y \in X$.

(ii) $d(x, y)=0$ if and only if $x=y$.

(iii) $d(x, y)=d(y, x)$ for all $x, y \in X$.

(iv) $d(x, y)+d(y, z) \geq d(x, z)$ for all $x, y, z \in X$. (This is called the triangle inequality after the result in Euclidean geometry that the sum of the lengths of two sides of a triangle is at least as great as the length of the third side.)

Then we say that $d$ is a metric on $X$ and that $(X, d)$ is a metric space.

You should imagine the author muttering under his breath

(i) Distances are always positive.

(ii) Two points are zero distance apart if and only if they are the same point.

(iii) The distance from $A$ to $B$ is the same as the distance from $B$ to $A$.

(iv) The distance from $A$ to $B$ via $C$ is at least as great as the distance from $A$ to $B$ directly.'

Exercise 2.2. If $d: X^{2} \rightarrow \mathbb{R}$ is a function with the following properties:-

\footnotetext{
${ }^{1}$ We thus allow $X=\varnothing$. This is purely a question of taste. If we did not allow this possibility, then, every time we defined a metric space $(X, d)$, we would need to prove that $X$ was non-empty. If we do allow this possibility, and we prefer to reason about non-empty spaces, then we can begin our proof with the words 'If $X$ is empty, then the result is vacuously true, so we may assume that $X$ is non-empty.' (Of course, the result may be false for $X=\varnothing$, in which case the statement of the theorem must include the condition $X \neq \varnothing$.)
} 
(ii) $d(x, y)=0$ if and only if $x=y$,

(iii) $d(x, y)=d(y, x)$ for all $x, y \in X$,

(iv) $d(x, y)+d(y, z) \geq d(x, z)$ for all $x, y, z \in X$,

show that $d$ is a metric on $X$.

[Thus condition (i) of the definition is redundant.]

Solution. See page 57 for a solution.

Exercise 2.3. Let $X$ be the set of towns on the British railway system. Consider the $d$ corresponding to the examples (1) to (4) and discuss informally whether conditions (i) to (iv) apply.

[An open ended question like this will be more useful if tackled in a spirit of good will.]

Exercise 2.4. Let $X=\{a, b, c\}$ with $a, b$ and $c$ distinct. Write down functions $d_{j}: X^{2} \rightarrow \mathbb{R}$ satisfying condition (i) of Definition 2.1 such that:-

(1) $d_{1}$ satisfies conditions (ii) and (iii) but not (iv).

(2) $d_{2}$ satisfies conditions (iii) and (iv) and $d_{2}(x, y)=0$ implies $x=y$, but it is not true that $x=y$ implies $d_{2}(x, y)=0$.

(3) $d_{3}$ satisfies conditions (iii) and (iv) and $x=y$ implies $d_{3}(x, y)=0$. but it is not true that $d_{3}(x, y)=0$ implies $x=y$.

(4) $d_{4}$ satisfies conditions (ii) and (iv) but not (iii).

You should verify your statements.

Solution. See page 58.

We give another axiom grubbing exercise as Exercise 15.1.

Exercise 2.5. Let $X$ be a set and $\rho: X^{2} \rightarrow \mathbb{R}$ a function with the following properties.

(i) $\rho(x, y) \geq 0$ for all $x, y \in X$.

(ii) $\rho(x, y)=0$ if and only if $x=y$.

(iv) $\rho(x, y)+\rho(y, z) \geq \rho(x, z)$ for all $x, y, z \in X$.

Show that, if we set $d(x, y)=\rho(x, y)+\rho(y, x)$, then $(X, d)$ is a metric space.

Here are some examples of metric spaces. You have met (or you will meet) the concept of a normed vector space both in algebra and analysis courses.

Definition 2.6. Let $V$ be a vector space over $\mathbb{F}$ (with $\mathbb{F}=\mathbb{R}$ or $\mathbb{F}=\mathbb{C}$ ) and $N: V \rightarrow \mathbb{R}$ a map such that, writing $N(\mathbf{u})=\|\mathbf{u}\|$, the following results hold.

(i) $\|\mathbf{u}\| \geq 0$ for all $\mathbf{u} \in V$.

(ii) If $\|\mathbf{u}\|=0$, then $\mathbf{u}=\mathbf{0}$.

(iii) If $\lambda \in \mathbb{F}$ and $\mathbf{u} \in V$, then $\|\lambda \mathbf{u}\|=|\lambda|\|\mathbf{u}\|$. 
(iv) [Triangle law.] If $\mathbf{u}, \mathbf{v} \in V$, then $\|\mathbf{u}\|+\|\mathbf{v}\| \geq\|\mathbf{u}+\mathbf{v}\|$.

Then we call \| $\|$ a norm and say that $(V,\|\|)$ is a normed vector space.

Exercise 2.7. By putting $\lambda=0$ in Definition 2.6 (iii), show that $\|\mathbf{0}\|=0$.

Any normed vector space can be made into a metric space in a natural way.

Lemma 2.8. If $(V,\|\|)$ is a normed vector space, then the condition

$$
d(\mathbf{u}, \mathbf{v})=\|\mathbf{u}-\mathbf{v}\|
$$

defines a metric d on $V$.

Proof. The easy proof is given on page 58.

Many metrics that we meet in analysis arise in this way.

However, not all metrics can be derived from norms. Here is a metric that turns out to be more important and less peculiar than it looks at first sight.

Definition 2.9. If $X$ is a set and we define $d: X^{2} \rightarrow \mathbb{R}$ by

$$
d(x, y)= \begin{cases}0 & \text { if } x=y \\ 1 & \text { if } x \neq y\end{cases}
$$

then $d$ is called the discrete metric on $X$.

Lemma 2.10. The discrete metric on $X$ is indeed a metric.

Proof. The easy proof is given on page 59 .

Exercise 2.11. (We deal with the matter somewhat better in Exercise 4.6) (i) If $V$ is a vector space over $\mathbb{R}$ and $d$ is a metric derived from a norm in the manner described above, then, if $\mathbf{u} \in V$ we have $d(\mathbf{0}, 2 \mathbf{u})=2 d(\mathbf{0}, \mathbf{u})$.

(ii) If $V$ is non-trivial (i.e. not zero-dimensional) vector space over $\mathbb{R}$ and $d$ is the discrete metric on $V$, then $d$ cannot be derived from a norm on $V$.

In algebra you have learnt (or you will learn) about inner product spaces. You have learnt (or you will learn) that every inner product gives rise to a norm in a natural way. Most norms in analysis do not arise in this way ${ }^{2}$, but the few that do are very important.

\footnotetext{
${ }^{2}$ This is not part of the course, but see Exercise 15.2 if you are interested.
} 
Definition 2.12. If $\mathrm{x} \in \mathbb{R}^{n}$, we write

$$
\|\mathbf{x}\|_{2}=\left(\sum_{j=1}^{n} x_{j}^{2}\right)^{1 / 2}
$$

where the positive square root is taken. We call \|\|$_{2}$ the Euclidean norm on $\mathbb{R}^{n}$.

The reader should prove the next lemma before proceeding further. If she cannot prove it she should be revising earlier courses rather than reading about new ones. (Pay particular attention to the triangle inequality. In my opinion, the easiest proof uses inner products but this is only an opinion and you may ignore it.).

Lemma 2.13. The Euclidean norm on $\mathbb{R}^{n}$ is indeed a norm.

The metric derived from the Euclidean norm is called the Euclidean metric. You should test any putative theorems on metric spaces on both $\mathbb{R}^{n}$ with the Euclidean metric and $\mathbb{R}^{n}$ with the discrete metric.

Exercise 2.14. [The counting metric.] If $E$ is a finite set and $\mathcal{E}$ is the collection of subsets of $E$, we write card $C$ for the number of elements in $C$ and

$$
d(A, B)=\operatorname{card} A \triangle B .
$$

Show that $d$ is a metric on $\mathcal{E}$. The reader may be inclined to dismiss this metric as uninteresting but it plays an important role (as the Hamming metric) in the Part II course Codes and Cryptography.

Here are two metrics which are included simply to show that metrics do not have to be as simple as the ones above. I shall use them as examples once or twice, but they do not form part of standard mathematical knowledge and you do not have to learn their definition.

Definition 2.15. (i) If we define $d: \mathbb{R}^{2} \times \mathbb{R}^{2} \rightarrow \mathbb{R}$ by

$$
d(\mathbf{u}, \mathbf{v})= \begin{cases}\|\mathbf{u}\|_{2}+\|\mathbf{v}\|_{2}, & \text { if } \mathbf{u} \neq \mathbf{v} \\ 0 & \text { if } \mathbf{u}=\mathbf{v}\end{cases}
$$

then $d$ is called the British Rail express metric. (To get from $A$ to $B$ travel via London.)

(ii) If we define $d: \mathbb{R}^{2} \times \mathbb{R}^{2} \rightarrow \mathbb{R}$ by

$$
d(\mathbf{u}, \mathbf{v})= \begin{cases}\|\mathbf{u}-\mathbf{v}\|_{2} & \text { if } \mathbf{u} \text { and } \mathbf{v} \text { are linearly dependent } \\ \|\mathbf{u}\|_{2}+\|\mathbf{v}\|_{2} & \text { otherwise }\end{cases}
$$


then $d$ is called the British Rail stopping metric. (To get from $A$ to $B$ travel via London unless $A$ and $B$ are on the same London route.)

(Recall that $\mathbf{u}$ and $\mathbf{v}$ are linearly dependent if $\mathbf{u}=\lambda \mathbf{v}$ for some real $\lambda$ and/or $\mathbf{v}=\mathbf{0}$.)

Exercise 2.16. Show that the British Rail express metric and the British Rail stopping metric are indeed metrics.

Solution. On page 59 we show that the British Rail stopping metric is indeed a metric. The British Rail express metric can be dealt with similarly.

\section{Continuity and open sets for metric spaces}

Some definitions and results transfer essentially unchanged from classical analysis on $\mathbb{R}$ to metric spaces. Recall the classical definition of continuity.

Definition 3.1. [Old definition.] A function $f: \mathbb{R} \rightarrow \mathbb{R}$ is called continuous if, given $t \in \mathbb{R}$ and $\epsilon>0$, we can find a $\delta(t, \epsilon)>0$ such that

$$
|f(t)-f(s)|<\epsilon \text { whenever }|t-s|<\delta(t, \epsilon) .
$$

It is not hard to extend this definition to our new, wider context.

Definition 3.2. [New definition.] Let $(X, d)$ and $(Y, \rho)$ be metric spaces. A function $f: X \rightarrow Y$ is called continuous if, given $t \in X$ and $\epsilon>0$, we can find $a \delta(t, \epsilon)>0$ such that

$$
\rho(f(t), f(s))<\epsilon \text { whenever } d(t, s)<\delta(t, \epsilon) .
$$

It may help you grasp this definition if you read ' $\rho(f(t), f(s))$ ' as 'the distance from $f(t)$ to $f(s)$ in $Y$ ' and ' $d(t, s)$ ' as 'the distance from $t$ to $s$ in $X^{\prime}$.

Lemma 3.3. [The composition law.] If $(X, d)$ and $(Y, \rho)$ and $(Z, \sigma)$ are metric spaces and $g: X \rightarrow Y, f: Y \rightarrow Z$ are continuous, then so is the composition $f g$.

Proof. This is identical to the one we met in classical analysis. If needed, details are given on page 60 .

Exercise 3.4. Let $\mathbb{R}$ and $\mathbb{R}^{2}$ have their usual (Euclidean) metric.

(i) Suppose that $f: \mathbb{R} \rightarrow \mathbb{R}$ and $g: \mathbb{R} \rightarrow \mathbb{R}$ are continuous. Show that the $\operatorname{map}(f, g): \mathbb{R}^{2} \rightarrow \mathbb{R}^{2}$ is continuous.

(ii) Show that the map $M: \mathbb{R}^{2} \rightarrow \mathbb{R}$ given by $M(x, y)=x y$ is continuous.

(iii) Use the composition law to show that the map $m: \mathbb{R}^{2} \rightarrow \mathbb{R}$ given by $m(x, y)=f(x) g(y)$ is continuous. 
Solution. See page 60.

Exercise 3.4 may look perverse at first sight, but, in fact, we usually show functions to be continuous by considering them as compositions of simpler functions rather than using the definition directly. Think about

$$
x \mapsto \log \left(2+\sin \frac{1}{1+x^{2}}\right) .
$$

If you are interested, we continue the chain of thought in Exercise 15.3. If you are not interested or are mildly confused by all this, just ignore this paragraph.

Just as there are 'well behaved' and 'badly behaved' functions between spaces, so there are 'well behaved' and 'badly behaved' subsets of spaces. In classical analysis and analysis on metric spaces the notion of continuous function is sufficiently wide to give us a large collection of interesting functions and sufficiently narrow to ensure reasonable behaviour ${ }^{3}$. In introductory analysis we work on $\mathbb{R}$ with the Euclidean metric and only consider subsets in the form of intervals. Once we move to $\mathbb{R}^{2}$ with the Euclidean metric, it becomes clear that there is no appropriate analogue to intervals. (We want appropriate rectangles to be well behaved, but we also want to talk about discs and triangles and blobs.)

Cantor identified two particular classes of 'well behaved' sets. We start with open sets.

Definition 3.5. Let $(X, d)$ be a metric space. We say that a subset $E$ is open in $X$ if, whenever $e \in E$, we can find $a \delta>0$ (depending on e) such that

$$
x \in E \text { whenever } d(x, e)<\delta .
$$

Suppose we work in $\mathbb{R}^{2}$ with the Euclidean metric. If $E$ is an open set then any point $\mathbf{e}$ in $E$ is the centre of a disc of strictly positive radius all of whose points lie in $E$. If we are sufficiently short sighted, every point that we can see from e lies in $E$. This property turns out to be a key to many proofs in classical analysis (remember that in the proof of Rolle's theorem it was vital that the maximum did not lie at an end point) and complex analysis (where we examine functions analytic on an open set).

Here are a couple of simple examples of an open set and a simple example of a set which is not open.

\footnotetext{
${ }^{3}$ Sentences like this are not mathematical statements, but many mathematicians find them useful.
} 
Example 3.6. (i) Let $(X, d)$ be a metric space. If $r>0$, then

$$
B(x, r)=\{y: d(x, y)<r\}
$$

is open.

(ii) If we work in $\mathbb{R}^{n}$ with the Euclidean metric, then the one point set $\{\mathbf{x}\}$ is not open.

(iii) If $(X, d)$ is a discrete metric space, then

$$
\{x\}=B(x, 1 / 2)
$$

and all subsets of $X$ are open.

Proof. See page 61.

We call $B(\mathbf{x}, r)$ the open ball with centre $\mathbf{x}$ and radius $r$. The following result is very important for the course, but is also very easy to check.

Theorem 3.7. If $(X, d)$ is a metric space, then the following statements are true.

(i) The empty set $\varnothing$ and the space $X$ are open.

(ii) If $U_{\alpha}$ is open for all $\alpha \in A$, then $\bigcup_{\alpha \in A} U_{\alpha}$ is open. (In other words, the union of open sets is open.)

(iii) If $U_{j}$ is open for all $1 \leq j \leq n$, then $\bigcap_{j=1}^{n} U_{j}$ is open.

Proof. See page 62.

It is important to realise that we place no restriction on the size of $A$ in (ii). In particular, $A$ could be uncountable. However, conclusion (iii) cannot be extended.

Example 3.8. Let us work in $\mathbb{R}^{n}$ with the usual metric. Then $B(\mathbf{x}, 1 / j)$ is open, but $\bigcap_{j=1}^{\infty} B(\mathbf{x}, 1 / j)=\{\mathbf{x}\}$ is not.

Proof. See Example 3.6.

There is a remarkable connection between the notion of open sets and continuity.

Theorem 3.9. Let $(X, d)$ and $(Y, \rho)$ be metric spaces. A function $f: X \rightarrow Y$ is continuous if and only if $f^{-1}(U)$ is open in $X$ whenever $U$ is open in $Y$.

Proof. See page 63.

Note that the theorem does not work 'in the opposite direction'. 
Example 3.10. Let $X=\mathbb{R}$ and $d$ be the discrete metric. Let $Y=\mathbb{R}$ and $\rho$ be the usual (Euclidean) metric.

(i) If we define $f: X \rightarrow Y$ by $f(x)=x$, then $f$ is continuous but there exist open sets $U$ in $X$ such that $f(U)$ is not open.

(ii) If we define $g: Y \rightarrow X$ by $g(y)=y$, then $g$ is not continuous but $g(V)$ is open in $X$ whenever $V$ is open in $Y$.

Proof. Very easy, but see page 63 if you need.

The message of this example is reinforced by the more complicated Exercise 15.4.

Observe that Theorem 3.9 gives a very neat proof of the composition law. Theorem 3.3. If $(X, d),(Y, \rho),(Z, \sigma)$ are metric spaces and $g: X \rightarrow Y$, $f: Y \rightarrow Z$ are continuous, then so is the composition $f g$.

New proof. If $U$ is open in $Z$, then, by continuity, $f^{-1}(U)$ is open in $Y$ and so, by continuity, $(f g)^{-1}(U)=g^{-1}\left(f^{-1}(U)\right)$ is open in $X$. Thus $f g$ is continuous.

This confirms our feeling that the ideas of this chapter are on the right track.

We finish with an exercise, which may be omitted at first reading, but which should be done at some time as examples of what open sets can look like.

Exercise 3.11. Consider $\mathbb{R}^{2}$. For each of the British rail express and British rail stopping metrics:-

(i) Describe the open balls. (Consider both large and small radii.)

(ii) Describe the open sets as well as you can. (There is a nice description for the British rail express metric.) Give reasons for your answers.

Solution. See page 64 .

\section{Closed sets for metric spaces}

The second class of well behaved sets identified by Cantor were the closed sets. In order to define closed sets in metric spaces, we need a notion of limit. Fortunately, the classical definition generalises without difficulty.

Definition 4.1. Consider a sequence $x_{n}$ in a metric space $(X, d)$. If $x \in X$ and, given $\epsilon>0$, we can find an integer $N \geq 1$ (depending on $\epsilon$ ) such that

$$
d\left(x_{n}, x\right)<\epsilon \text { for all } n \geq N
$$


then we say that $x_{n} \rightarrow x$ as $n \rightarrow \infty$ and that $x$ is the limit of the sequence $x_{n}$.

Lemma 4.2. Consider a metric space $(X, d)$. If a sequence $x_{n}$ has a limit, then that limit is unique.

Proof. The simple proof is given on page 65. Just as in the next exercise, it suffices to follow the 'first course in analysis' proof with minimal changes.

Exercise 4.3. Consider two metric spaces $(X, d)$ and $(Y, \rho)$. Show that a function $f: X \rightarrow Y$ is continuous if and only if, whenever $x_{n} \in X$ and $x_{n} \rightarrow x$ as $n \rightarrow \infty$, we have $f\left(x_{n}\right) \rightarrow f(x)$

Solution. See page 65, if necessary.

Definition 4.4. Let $(X, d)$ be a metric space. A set $F$ in $X$ is said to be closed if, whenever $x_{n} \in F$ and $x_{n} \rightarrow x$ as $n \rightarrow \infty$, it follows that $x \in F$.

The following exercises are easy, but instructive.

Exercise 4.5. (i) If $(X, d)$ is any metric space, then $X$ and $\varnothing$ are both open and closed.

(ii) If we consider $\mathbb{R}$ with the usual metric and take $b>a$, then $[a, b]$ is closed but not open, $(a, b)$ is open but not closed and $[a, b)$ is neither open nor closed.

Exercise 4.6. (i) If $(X, d)$ is a metric space with discrete metric $d$, then all subsets of $X$ are both open and closed.

(ii) If $V$ is a vector space over $\mathbb{R}$ and $d$ is a metric derived from a norm, show that the one point sets $\{\mathbf{x}\}$ are not open. Deduce that $d$ cannot be derived from a norm on $V$.

It is easy to see why closed sets will be useful in those parts of analysis which involve taking limits. The reader will recall theorems in elementary analysis (for example the boundedness of continuous functions) which were true for closed intervals, but not for other types of intervals.

Life is made much easier by the very close link between the notions of closed and open sets given by our next theorem.

Theorem 4.7. Let $(X, d)$ be a metric space. A set $F$ in $X$ is closed if and only if its complement is open.

Proof. We give the proof on page 66 . 
We can now deduce properties of closed sets from properties of open sets by complementation. In particular, we have the following complementary versions of Theorems 3.7 and 3.9

Theorem 4.8. If $(X, d)$ is a metric space, then the following statements are true.

(i) The empty set $\varnothing$ and the space $X$ are closed.

(ii) If $F_{\alpha}$ is closed for all $\alpha \in A$, then $\bigcap_{\alpha \in A} F_{\alpha}$ is closed. (In other words the intersection of closed sets is closed.)

(iii) If $F_{j}$ is closed for all $1 \leq j \leq n$, then $\bigcup_{j=1}^{n} F_{j}$ is closed.

Proof. See page 66.

Theorem 4.9. Let $(X, d)$ and $(Y, \rho)$ be metric spaces. A function $f: X \rightarrow Y$ is continuous if and only if $f^{-1}(F)$ is closed in $X$ whenever $F$ is closed in $Y$.

Proof. See page 66.

\section{Topological spaces}

We now investigate general objects which have the structure described by Theorem 3.7.

Definition 5.1. Let $X$ be a set and $\tau$ a collection of subsets of $X$ with the following properties.

(i) The empty set $\varnothing \in \tau$ and the space $X \in \tau$.

(ii) If $U_{\alpha} \in \tau$ for all $\alpha \in A$, then $\bigcup_{\alpha \in A} U_{\alpha} \in \tau$.

(iii) If $U_{j} \in \tau$ for all $1 \leq j \leq n$, then $\bigcap_{j=1}^{n} U_{j} \in \tau$.

Then we say that $\tau$ is a topology on $X$ and that $(X, \tau)$ is a topological space.

Theorem 5.2. If $(X, d)$ is a metric space, then the collection of open sets forms a topology.

Proof. This is Theorem 3.7.

If $(X, d)$ is a metric space we call the collection of open sets the topology induced by the metric.

If $(X, \tau)$ is a topological space we extend the notion of open set by calling the members of $\tau$ open sets. The discussion above ensures what computer scientists call 'downward compatibility'. 
Exercise 5.3. If $(X, d)$ is a metric space with the discrete metric, show that the induced topology consists of all the subsets of $X$.

We call the topology consisting of all subsets of $X$ the discrete topology on $X$.

Exercise 5.4. If $X$ is a set and $\tau=\{\varnothing, X\}$, then $\tau$ is a topology.

We call $\{\varnothing, X\}$ the indiscrete topology on $X$.

Exercise 5.5. (i) If $F$ is a finite set and $(F, d)$ is a metric space, show that the induced topology is the discrete topology.

(ii) If $F$ is a finite set with more than one point, show that the indiscrete topology is not induced by any metric.

You should test any putative theorems on topological spaces on the discrete topology and the indiscrete topology, $\mathbb{R}^{n}$ with the topology derived from the Euclidean metric and $[0,1]$ with the topology derived from the Euclidean metric.

The following exercise is tedious but instructive (the tediousness is the instruction).

Exercise 5.6. Write $\mathcal{P}(Y)$ for the collection of subsets of $Y$. If $X$ has three elements, how many elements does $\mathcal{P}(\mathcal{P}(X))$ have?

How many topologies are there on $X$ ?

Solution. See page 67.

The idea of downward compatibility suggests 'turning Theorem 3.9 in a definition'.

Definition 5.7. Let $(X, \tau)$ and $(Y, \sigma)$ be topological spaces. A function $f$ : $X \rightarrow Y$ is said to be continuous if and only if $f^{-1}(U)$ is open in $X$ whenever $U$ is open in $Y$.

Theorem 3.9 tells us that if $(X, d)$ and $(Y, \rho)$ are metric spaces the notion of a continuous function $f: X \rightarrow Y$ is the same whether we consider the metrics or the topologies derived from them.

The proof of Theorem 3.3 given on page 10 carries over unchanged to give the following generalisation.

Theorem 5.8. If $(X, \tau),(Y, \sigma),(Z, \mu)$ are topological spaces and $g: X \rightarrow Y$, $f: Y \rightarrow Z$ are continuous, then so is the composition $f g$.

Downward compatibility suggests the definition of a closed set for a topological space based on Theorem 4.7. 
Definition 5.9. Let $(X, \tau)$ be a topological space. A set $F$ in $X$ is said to be closed if its complement is open.

Theorem 4.7 tells us that if $(X, d)$ is a metric space the notion of a closed set is the same whether we consider the metric or the topology derived from it.

Just as in the metric case, we can deduce properties of closed sets from properties of open sets by complementation. In particular, the same proofs as we gave in the metric case give the following extensions of Theorems 4.8 and 4.9

Theorem 5.10. If $(X, \tau)$ is a topological space, then the following statements are true.

(i) The empty set $\varnothing$ and the space $X$ are closed.

(ii) If $F_{\alpha}$ is closed for all $\alpha \in A$, then $\bigcap_{\alpha \in A} F_{\alpha}$ is closed. (In other words, the intersection of closed sets is closed.)

(iii) If $F_{j}$ is closed for all $1 \leq j \leq n$, then $\bigcup_{j=1}^{n} F_{j}$ is closed.

Theorem 5.11. Let $(X, \tau)$ and $(Y, \sigma)$ be topological spaces. A function $f$ : $X \rightarrow Y$ is continuous if and only if $f^{-1}(F)$ is closed in $X$ whenever $F$ is closed in $Y$.

\section{$6 \quad$ Interior and closure}

The next section is short, not because the ideas are unimportant, but because they are so useful that the reader will meet them over and over again in other courses.

Definition 6.1. Let $(X, \tau)$ be a topological space and $A$ a subset of $X$. We write

$$
\operatorname{Int} A=\bigcup\{U \in \tau: U \subseteq A\} \text { and } \mathrm{Cl} A=\bigcap\{F \text { closed }: F \supseteq A\}
$$

and call $\mathrm{Cl} A$ the closure of $A$ and $\operatorname{Int} A$ the interior of $A$.

Simple complementation, which I leave to the reader, shows how closely the two notions of closure and interior are related. (Recall that $A^{c}=X \backslash A$, the complement of $A$.)

Lemma 6.2. With the notation of Definition 6.1

$$
\left(\mathrm{Cl} A^{c}\right)^{c}=\operatorname{Int} A \text { and }\left(\operatorname{Int} A^{c}\right)^{c}=\mathrm{Cl} A .
$$


There are other useful ways of viewing $\operatorname{Int} A$ and $\mathrm{Cl} A$.

Lemma 6.3. Let $(X, \tau)$ be a topological space and $A$ a subset of $X$.

(i) Int $A=\{x \in A: \exists U \in \tau$ with $x \in U \subseteq A\}$.

(ii) Int $A$ is the unique $V \in \tau$ such that $V \subseteq A$ and, if $W \in \tau$ and $V \subseteq W \subseteq A$, then $V=W$. (Informally, Int $A$ is the largest open set contained in $A$.)

Proof. The easy proof is given on page 67 .

Simple complementation, which I leave to the reader, gives the corresponding results for closure.

Lemma 6.4. Let $(X, \tau)$ be a topological space and $A$ a subset of $X$.

(i) $\mathrm{Cl} A=\{x \in A: \forall U \in \tau$ with $x \in U$, we have $A \cap U \neq \varnothing\}$.

(ii) $\mathrm{Cl} A$ is the unique closed set $G$ such that $G \supseteq A$ and, if $F$ is closed with $G \supseteq F \supseteq A$, then $F=G$. (Informally, $\mathrm{Cl} A$ is the smallest closed set containing $A$.)

Exercise 6.5. Prove Lemma 6.4 directly without using Lemma 6.3.

Sometimes, when touring an ancient college, you may be shown a 14th century wall which still plays an important part in holding up the building. The next lemma goes back to Cantor and the very beginnings of topology. (It would then have been a definition rather than a lemma.)

Lemma 6.6. Let $(X, d)$ be a metric space and $A$ a subset of $X$. Then $\mathrm{Cl} A$ consists of all those $x$ such that we can find $x_{n} \in A$ with $d\left(x, x_{n}\right) \rightarrow 0$. (In old fashioned terminology, the closure of $A$ is its set of limit points.)

Proof. The easy proof is given on page 68 .

The idea of closure is strongly linked to the idea of a dense subset.

Definition 6.7. Let $(X, \tau)$ be a topological space and $F$ a closed subset of $X$. We say that $A \subseteq X$ is dense subset of $F$ if $\mathrm{Cl} A=F$.

In some sense $A$ is a 'skeleton' of $F$ and we may hope to prove results about $F$ by first proving them on the dense subset $A$ and then extending the result by 'density'. Sometimes this idea works (see, for example, part (i) of Exercise 6.8) and sometimes it does not (see, for example, part (ii) of Exercise 6.8). When it does work, this is very powerful technique. 
Exercise 6.8. (i) Let $(X, \tau)$ be a topological space and $(Y, d)$ a metric space ${ }^{4}$. If $f, g: X \rightarrow Y$ are continuous and $f(x)=g(x)$ for all $x \in A$, where $A$ is dense in $X$, show that $f(x)=g(x)$ for all $x \in X$.

(ii) Consider the unit interval [0,1] with the Euclidean metric and $A=$ $[0,1] \cap \mathbb{Q}$ with the inherited metric. Exhibit, with proof, a continuous map $f: A \rightarrow \mathbb{R}$ (where $\mathbb{R}$ has the standard metric) such that there does not exist a continuous map $\tilde{f}:[0,1] \rightarrow \mathbb{R}$ with $\tilde{f}(x)=f(x)$ for all $x \in A$.

Solution. We give the solution on Page 18

\section{More on topological structures}

Two groups are the same for the purposes of group theory if they are (group) isomorphic. Two vector spaces are the same for the purposes of linear algebra if they are (vector space) isomorphic. When are two topological spaces $(X, \tau)$ and $(Y, \sigma)$ the same for the purposes of topology? In other words, when does there exist a bijection between $X$ and $Y$ in which open sets correspond to open sets, and the grammar of topology (things like union and inclusion) is preserved? A little reflection shows that the next definition provides the answer we want. (Exercise 15.8 is vaguely relevant.)

Definition 7.1. We say that two topological spaces $(X, \tau)$ and $(Y, \sigma)$ are homeomorphic if there exists a bijection $\theta: X \rightarrow Y$ such that $\theta$ and $\theta^{-1}$ are continuous. We call $\theta$ a homeomorphism.

The following exercise acts as useful revision of concepts learnt last year.

Exercise 7.2. Show that homeomorphism is an equivalence relation on topological spaces.

Homeomorphism only implies equivalence for the purposes of topology. To emphasise this, we introduce a couple of related ideas which are fundamental to analysis on metric spaces, but which will only be referred to here in this course.

Definition 7.3. (i) If $(X, d)$ is a metric space, we say that a sequence $x_{n}$ in $X$ is Cauchy if, given $\epsilon>0$, we can find an $N_{0}(\epsilon)$ with

$$
d\left(x_{n}, x_{m}\right)<\epsilon \text { whenever } n, m \geq N_{0}(\epsilon) .
$$

(ii) We say that a metric space $(X, d)$ is complete if every Cauchy sequence converges.

\footnotetext{
${ }^{4}$ We give a slight improvement in Exercise 8.7.
} 
Example 7.4. Let $X=\mathbb{R}$ and let $d$ be the usual metric on $\mathbb{R}$. Let $Y=(0,1)$ (the open interval with end points 0 and 1 ) and let $\rho$ be the usual metric on $(0,1)$. Then $(X, d)$ and $(Y, \rho)$ are homeomorphic as topological spaces, but $(X, d)$ is complete and $(Y, \rho)$ is not.

Proof. See page 69.

We say that 'completeness is not a topological property'. Exercise 16.31 shows that there exist metric spaces which are not homeomorphic to any complete metric space.

In group theory, we usually prove that two groups are isomorphic by constructing an explicit isomorphism and that two groups are not isomorphic by finding a group property exhibited by one but not by the other. Similarly, in topology, we usually prove that two topological spaces are homeomorphic by constructing an explicit homeomorphism and that two topological spaces are not homeomorphic by finding a topological property exhibited by one but not by the other. Later in this course we will meet some topological properties like being Hausdorff and compactness and you will be able to tackle Exercise 15.20.

We also want to be able to construct new topological spaces from old. To do this we we make use of a simple, but useful, lemma.

Lemma 7.5. Let $X$ be a space and let $\mathcal{H}$ be a collection of subsets of $X$. Then there exists a unique topology $\tau_{\mathcal{H}}$ such that

(i) $\tau_{\mathcal{H}} \supseteq \mathcal{H}$, and

(ii) if $\tau$ is a topology with $\tau \supseteq \mathcal{H}$, then $\tau \supseteq \tau_{\mathcal{H}}$.

Proof. The proof, which follows the standard pattern for such things, is given on page 69.

We call $\tau_{\mathcal{H}}$ the smallest (or coarsest) topology containing $\mathcal{H}$.

Lemma 7.6. Suppose that $A$ is non-empty and the spaces $\left(X_{\alpha}, \tau_{\alpha}\right)$ are topological spaces and we have maps $f_{\alpha}: X \rightarrow X_{\alpha}[\alpha \in A]$. Then there is a smallest topology $\tau$ on $X$ for which the maps $f_{\alpha}$ are continuous.

Proof. A topology $\sigma$ on $X$ makes all the $f_{\alpha}$ continuous if and only if it contains

$$
\mathcal{H}=\left\{f_{\alpha}^{-1}(U): U \in \tau_{\alpha}, \alpha \in A\right\} .
$$

Now apply Lemma 7.5.

Recall that, if $Y \subseteq X$, then the inclusion map $j: Y \rightarrow X$ is defined by $j(y)=y$ for all $y \in Y$. 
Definition 7.7. If $(X, \tau)$ is a topological space and $Y \subseteq X$ then the subspace topology $\tau_{Y}$ on $Y$ induced by $\tau$ is the smallest topology on $Y$ for which the inclusion map is continuous.

Lemma 7.8. If $(X, \tau)$ is a topological space and $Y \subseteq X$, then the subspace topology $\tau_{Y}$ on $Y$ is the collection of sets $Y \cap U$ with $U \in \tau$.

Proof. The very easy proof is given on page 70 .

Exercise 7.9. (i) If $(X, \tau)$ is a topological space and $Y \subseteq X$ is open, show that the subspace topology $\tau_{Y}$ on $Y$ is the collection of sets $U \in \tau$ with $U \subseteq Y$.

(ii) Consider $\mathbb{R}$ with the usual topology $\tau$ (that is, the topology derived from the Euclidean metric). If $Y=[0,1]$, show that $[0,1 / 2) \in \tau_{Y}$ but $[0,1 / 2) \notin \tau$.

Exercise 7.10. Let $(X, d)$ be a metric space, $Y$ a subset of $X$ and $d_{Y}$ the metric d restricted to $Y$ (formally, $d_{Y}: Y^{2} \rightarrow \mathbb{R}$ is given by $d_{Y}(x, y)=d(x, y)$ for $x, y \in Y$ ). Then if we give $X$ the topology induced by $d$, the subspace topology on $Y$ is identical with the topology induced by $d_{Y}$.

[This is an exercise in stating the obvious.]

Next recall that if $X$ and $Y$ are sets the projection maps $\pi_{X}: X \times Y \rightarrow X$ and $\pi_{Y}: X \times Y \rightarrow Y$ are given by

$$
\begin{aligned}
& \pi_{X}(x, y)=x \\
& \pi_{Y}(x, y)=y .
\end{aligned}
$$

Definition 7.11. If $(X, \tau)$ and $(Y, \sigma)$ are topological spaces, then the product topology $\mu$ on $X \times Y$ is the smallest topology on $X \times Y$ for which the projection maps $\pi_{X}$ and $\pi_{Y}$ are continuous.

Lemma 7.12. Let $(X, \tau)$ and $(Y, \sigma)$ be topological spaces and $\lambda$ the product topology on $X \times Y$. Then $O \in \lambda$ if and only if, given $(x, y) \in O$, we can find $U \in \tau$ and $V \in \sigma$ such that

$$
(x, y) \in U \times V \subseteq O .
$$

Proof. See page 70.

We give a slightly different treatment of the matter in Exercise 13.9.

The next remark is useful for proving results like those in Exercise 7.14. 
Lemma 7.13. Let $\tau_{1}$ and $\tau_{2}$ be two topologies on the same space $X$.

(i) We have $\tau_{1} \subseteq \tau_{2}$ if and only if, given $x \in U \in \tau_{1}$, we can find $V \in \tau_{2}$ such that $x \in V \subseteq U$.

(ii) We have $\tau_{1}=\tau_{2}$ if and only if, given $x \in U \in \tau_{1}$, we can find $V \in \tau_{2}$ such that $x \in V \subseteq U$ and, given $x \in U \in \tau_{2}$, we can find $V \in \tau_{1}$ such that $x \in V \subseteq U$.

Proof. The easy proof is given on Page 72

Exercise 7.14. Let $\left(X_{1}, d_{1}\right)$ and $\left(X_{2}, d_{2}\right)$ be metric spaces. Let $\tau$ be the product topology on $X_{1} \times X_{2}$ where $X_{j}$ is given the topology induced by $d_{j}$ $[j=1,2]$.

Define $\rho_{k}:\left(X_{1} \times X_{2}\right)^{2} \rightarrow \mathbb{R}$ by

$$
\begin{aligned}
& \rho_{1}((x, y),(u, v))=d_{1}(x, u), \\
& \rho_{2}((x, y),(u, v))=d_{1}(x, u)+d_{2}(y, v), \\
& \rho_{3}((x, y),(u, v))=\max \left(d_{1}(x, u), d_{2}(y, v)\right), \\
& \rho_{4}((x, y),(u, v))=\left(d_{1}(x, u)^{2}+d_{2}(y, v)^{2}\right)^{1 / 2} .
\end{aligned}
$$

Establish that $\rho_{1}$ is not a metric and that $\rho_{2}, \rho_{3}$ and $\rho_{4}$ are. Show that each of the $\rho_{j}$ with $2 \leq j \leq 4$ induces the product topology $\tau$ on $X_{1} \times X_{2}$.

It is easy to extend our definitions and results to any finite product of topological spaces ${ }^{5}$. In fact, it is not difficult to extend our definition to the product of an infinite collection of topological spaces, but I feel that it is important for the reader to concentrate on first thoroughly understanding the finite product case and I have relegated the infinite case to an exercise (Exercise 15.9).

We conclude this chapter by looking briefly at the quotient topology. This will not play a major part in our course and the reader should not worry too much about it.

If $\sim$ is an equivalence relation on a set $X$, then we know from previous courses that it gives rise to equivalence classes

$$
[x]=\{y \in X: y \sim x\} .
$$

There is a natural map $q$ from $X$ to the space $X / \sim$ of equivalence classes given by $q(x)=[x]$. When we defined the subspace and product topologies, we used natural maps from the new spaces to the old spaces. Here, we have

\footnotetext{
${ }^{5}$ Once you are confident with the material you may wish to look at Exercise 16.8, but this exercise is confusing for the beginner and trivial to the expert.
} 
a natural map from the old space to the new, so our definition has to take a different form.

Since intersection and union behave well under inverse mappings, it is easy to check the following statement.

Lemma 7.15. Let $(X, \tau)$ be a topological space and $\sim$ an equivalence relation on $X$. Write $q$ for the map from $X$ to the quotient space $X / \sim$ given by $q(x)=[x]$. Then

$$
\sigma=\left\{U \subseteq X / \sim: q^{-1}(U) \in \tau\right\}
$$

is a topology.

Definition 7.16. Under the assumptions and with the notation of Lemma 7.15 we call $\sigma$ the quotient topology on $X / \sim$.

The following is just a restatement of the definition.

Lemma 7.17. Under the assumptions and with the notation of Lemma 7.15, the quotient topology consists of the sets $U$ such that

$$
\bigcup_{[x] \in U}[x] \in \tau
$$

Later we shall give an example (Exercise 10.7) of a nice quotient topology. Exercise 15.24, which requires ideas from later in the course, is an example of really nasty quotient topology.

In general, the quotient topology can be extremely unpleasant (basically because equivalence relations form a very wide class) and although nice equivalence relations sometimes give very useful quotient topologies, you should always think before using one. Exercises 15.11 and 15.12 give some further information.

\section{Hausdorff spaces}

When we work in a metric space, we make repeated use of the fact that, if $d(x, y)=0$ then $x=y$. The metric is 'powerful enough to separate points'. The indiscrete topology, on the other hand, clearly cannot separate points.

When Hausdorff first crystallised the modern idea of a topological space, he included an extra condition to ensure 'separation of points'. It was later discovered that topologies without this extra condition could be useful, so it is now considered separately. 
Definition 8.1. A topological space $(X, \tau)$ is called Hausdorff if, whenever $x, y \in X$ and $x \neq y$, we can find $U, V \in \tau$ such that $x \in U, y \in V$ and $U \cap V=\varnothing$.

In the English educational system, it is traditional to draw $U$ and $V$ as little huts containing $x$ and $y$ and to say that $x$ and $y$ are 'housed off from each other'.

The next exercise requires a one line answer, but you should write that line down.

Exercise 8.2. Show that, if $(X, d)$ is a metric space, then the derived topology is Hausdorff.

Although we defer the discussion of neighbourhoods in general to towards the end of the course, it is natural to introduce the following locution here.

Definition 8.3. If $(X, \tau)$ is a topological space and $x \in U \in \tau$, we call $U$ an open neighbourhood of $x$.

Exercise 8.4. If $(X, \tau)$ is a topological space, then a subset $A$ of $X$ is open if and only if every point of $A$ has an open neighbourhood $U \subseteq A$.

Proof. The easy proof is given on page 72 .

Lemma 8.5. If $(X, \tau)$ is a Hausdorff space, then the one point sets $\{x\}$ are closed.

Proof. The easy proof is given on page 72 .

The following exercise shows that the converse to Lemma 8.5 is false and that, if we are to acquire any intuition about topological spaces, we will need to study a wide range of examples.

Exercise 8.6. Let $X$ be infinite (we could take $X=\mathbb{Z}$ or $X=\mathbb{R}$ ). We say that a subset $E$ of $X$ lies in $\tau$ if either $E=\varnothing$ or $X \backslash E$ is finite. Show that $\tau$ is a topology and that every one point set $\{x\}$ is closed but that $(X, \tau)$ is not Hausdorff.

What happens if $X$ is finite?

Solution. See page 73.

Exercise 8.7. Prove Exercise 6.8 (i) with ' $(Y, d)$ a metric space' replaced by ' $(Y, \sigma)$ a Hausdorff topological space'. 
It is easy to give examples of topologies which are not derived from metrics. It is somewhat harder to give examples of Hausdorff topologies which are not derived from metrics. An example is given in Exercise 15.13.

The next two lemmas are very useful.

Lemma 8.8. If $(X, \tau)$ is a Hausdorff topological space and $Y \subseteq X$, then $Y$ with the subspace topology is also Hausdorff.

Proof. The easy proof is given on page 73 .

Lemma 8.9. If $(X, \tau)$ and $(Y, \sigma)$ are Hausdorff topological spaces, then $X \times Y$ with the product topology is also Hausdorff.

Proof. The proof is easy (but there is one place where you can make a silly mistake). We give it on page 73 .

Exercise 15.12 shows that, even when the original topology is Hausdorff, the resulting quotient topology need not be.

\section{Compactness}

Halmos says somewhere that if an idea is used once it is a trick, if used twice it is a method, if used three times a theorem but if used four times it becomes an axiom.

Several important theorems in analysis hold for closed bounded intervals. Heine used a particular idea to prove one of these. Borel isolated the idea as a theorem (the Heine-Borel theorem), essentially Theorem 9.5 below. Many treatments of analysis (for example, Hardy's Pure Mathematics) use the Heine-Borel theorem as a basic tool. The notion of compactness represents the last stage in in the Halmos progression.

Definition 9.1. A topological space $(X, \tau)$ is called compact if, whenever we have a collection $U_{\alpha}$ of open sets $[\alpha \in A]$ with $\bigcup_{\alpha \in A} U_{\alpha}=X$, we can find a finite subcollection $U_{\alpha(1)}, U_{\alpha(2)}, \ldots, U_{\alpha(n)}$ with $\alpha(j) \in A[1 \leq j \leq n]$ such that $\bigcup_{j=1}^{n} U_{\alpha(j)}=X$.

Definition 9.2. If $(X, \tau)$ is a topological space, then a subset $Y$ is called compact if the subspace topology on $Y$ is compact.

The reader should have no difficulty in combining these two definitions to come up with the following restatement, 
Lemma 9.3. If $(X, \tau)$ is a topological space, then a subset $Y$ is compact if, whenever we have a collection $U_{\alpha}$ of open sets $[\alpha \in A]$ with $\bigcup_{\alpha} U_{\alpha} \supseteq Y$, we can find a finite subcollection $U_{\alpha(1)}, U_{\alpha(2)}, \ldots, U_{\alpha(n)}$ with $\alpha(j) \in A[1 \leq j \leq$ $n]$ such that $\bigcup_{j=1}^{n} U_{\alpha(j)} \supseteq Y$.

In other words, 'a set is compact if any cover by open sets has a finite subcover'.

The reader is warned that compactness is a subtle property which requires time and energy to master ${ }^{6}$. (At the simplest level, a substantial minority of examinees fail to get the definition correct.) Up to this point most of the proofs in this course have been simple deductions from definitions. Several of our theorems on compactness go much deeper and have quite intricate proofs.

Here are some simple examples of compactness and non-compactness.

Exercise 9.4. (i) Show that, if $X$ is finite, every topology on $X$ is compact.

(ii) Show that the discrete topology on a set $X$ is compact if and only if $X$ is finite.

(iii) Show that the indiscrete topology is always compact.

(iv) Show that the topology described in Exercise 8.6 is compact.

(v) Let $X$ be uncountable (we could take $X=\mathbb{R}$ ). We say that a subset $A$ of $X$ lies in $\tau$ if either $A=\varnothing$ or $X \backslash A$ is countable. Show that $\tau$ is a topology but that $(X, \tau)$ is not compact.

Solution. We give a partial solution for parts (iv) and (v) on page 74 .

We now come to our first major theorem.

Theorem 9.5. [The Heine-Borel Theorem.] Let $\mathbb{R}$ be given its usual topology (that is to say the topology derived from the usual Euclidean metric). Then the closed bounded interval $[a, b]$ is compact.

Proof. I give a hint on page 55 and a proof on 74 . An alternative proof, which is much less instructive, is given on page 32 .

Lemma 9.3 gives the following equivalent statement.

Theorem 9.6. Let $[a, b]$ be given its usual topology (that is to say the topology derived from the usual Euclidean metric). Then the derived topology is compact.

We now have a couple of very useful results.

\footnotetext{
${ }^{6}$ My generation only reached compactness after a long exposure to the classical HeineBorel theorem.
} 
Theorem 9.7. A closed subset of a compact set is compact. [More precisely, if $E$ is compact and $F$ closed in a given topology, then, if $F \subseteq E$, it follows that $F$ is compact.]

Proof. This is easy if you look at it the right way. See page 75.

Theorem 9.8. If $(X, \tau)$ is Hausdorff, then every compact set is closed.

Proof. This is harder, though it becomes easier if you realise that you must use the fact that $\tau$ is Hausdorff (see Example 9.9 below). We give a hint on page 55 and a proof on page 76 .

Example 9.9. Give an example of a topological space $(X, \tau)$ and a compact set in $X$ which is not closed.

Proof. There is a topological space with two points which will do. See page 76 .

Combining the Heine-Borel theorem with Theorems 9.7 and 9.8 and a little thought, we get a complete characterisation of the compact subsets of $\mathbb{R}$ (with the standard topology).

Theorem 9.10. Consider $(\mathbb{R}, \tau)$ with the standard (Euclidean) topology. A set $E$ is compact if and only if it is closed and bounded (that is to say, there exists a $M$ such that $|x| \leq M$ for all $x \in E$ ).

Proof. The easy proof is given on page 76 .

In Example 3.10 we saw that the continuous image of an open set need not be open. It also easy to see that the continuous image of a closed set need not be closed.

Exercise 9.11. Let $\mathbb{R}$ have the usual metric. Give an example of a continuous injective function $f: \mathbb{R} \rightarrow \mathbb{R}$ such that $f(\mathbb{R})$ is not closed.

Hint. Look at the solution of Example 7.4 if you need a hint.

However, the continuous image of a compact set is always compact.

Theorem 9.12. Let $(X, \tau)$ and $(Y, \sigma)$ be topological spaces and $f: X \rightarrow Y$ a continuous function. If $K$ is a compact subset of $X$, then $f(K)$ is a compact subset of $Y$.

Proof. This is easy if you look at it the right way. See page 77. 
This result has many delightful consequences. Recall, for example, that the quotient topology $X / \sim$ is defined in such a way that the quotient map $q: X \rightarrow X / \sim$ is continuous. Since $q(X)=X / \sim$, Theorem 9.12 gives us a positive property of the quotient topology.

Theorem 9.13. Let $(X, \tau)$ be a compact topological space and $\sim$ an equivalence relation on $X$. Then the quotient topology on $X / \sim$ is compact.

The next result follows at once from our characterisation of compact sets for the real line with the usual topology.

Theorem 9.14. Let $\mathbb{R}$ have the usual metric. If $K$ is a closed and bounded subset of $\mathbb{R}$ and $f: K \rightarrow \mathbb{R}$ is continuous, then $f(K)$ is closed and bounded.

This gives a striking extension of one of the crowning glories of a first course in analysis.

Theorem 9.15. Let $\mathbb{R}$ have the usual metric. If $K$ is a closed and bounded subset of $\mathbb{R}$ and $f: K \rightarrow \mathbb{R}$ is continuous, then $f$ is bounded and attains its bounds.

Proof. The straightforward proof is given on page 77 .

Theorem 9.15 is complemented by the following observation.

Exercise 9.16. Let $\mathbb{R}$ have the usual metric. If $K$ is subset of $\mathbb{R}$ with the property that, whenever $f: K \rightarrow \mathbb{R}$ is continuous, $f$ is bounded, it follows that $K$ is closed and bounded.

Proof. See page 78.

Theorem 9.15 has the following straightforward generalisation whose proof is left to the reader.

Theorem 9.17. If $K$ is a compact space and $f: K \rightarrow \mathbb{R}$ is continuous then $f$ is bounded and attains its bounds.

We also have the following useful result.

Theorem 9.18. Let $(X, \tau)$ be a compact and $(Y, \sigma)$ a Hausdorff topological space. If $f: X \rightarrow Y$ is a continuous bijection, then it is a homeomorphism.

Proof. There is a hint on page 56 and a proof on page 78 .

Theorem 9.18 is illuminated by the following almost trivial remark. 
Lemma 9.19. Let $\tau_{1}$ and $\tau_{2}$ be topologies on the same space $X$. The identity map

$$
\iota:\left(X, \tau_{1}\right) \rightarrow\left(X, \tau_{2}\right)
$$

from $X$ with topology $\tau_{1}$ to $X$ with topology $\tau_{2}$ given by $\iota(x)=x$ is continuous if and only if $\tau_{1} \supseteq \tau_{2}$.

Theorem 9.20. Let $\tau_{1}$ and $\tau_{2}$ be topologies on the same space $X$.

(i) If $\tau_{1} \supseteq \tau_{2}$ and $\tau_{1}$ is compact, then so is $\tau_{2}$.

(ii) If $\tau_{1} \supseteq \tau_{2}$ and $\tau_{2}$ is Hausdorff, then so is $\tau_{1}$.

(iii) If $\tau_{1} \supseteq \tau_{2}, \tau_{1}$ is compact and $\tau_{2}$ is Hausdorff, then $\tau_{1}=\tau_{2}$.

Proof. The routine proof is given on page 78 .

The reader may care to recall that 'Little Bear's porridge was neither too hot nor too cold but just right'.

With the hint given by the previous theorem it should be fairly easy to do do the next exercise.

Exercise 9.21. (i) Give an example of a Hausdorff space $(X, \tau)$ and a compact Hausdorff space $(Y, \sigma)$ together with a continuous bijection $f: X \rightarrow Y$ which is not a homeomorphism.

(ii) Give an example of a compact Hausdorff space $(X, \tau)$ and a compact space $(Y, \sigma)$ together with a continuous bijection $f: X \rightarrow Y$ which is not a homeomorphism.

Solution. See page 79.

We shall give a (not terribly convincing) example of the use of Theorem 9.18 in our proof of Exercise 10.7.

The reader may have gained the impression that compact Hausdorff spaces form an ideal backdrop for continuous functions to the reals. Later work shows that the impression is absolutely correct, but it must be remembered that many important spaces (including the real line with the usual topology) are not compact.

\section{Products of compact spaces}

The course contains one further major theorem on compactness.

Theorem 10.1. The product of two compact spaces is compact. (More formally, if $(X, \tau)$ and $(Y, \sigma)$ are compact topological spaces and $\lambda$ is the product topology, then $(X \times Y, \lambda)$ is compact. $)$ 
Proof. We give a very substantial hint on page 56 and a proof on page 79 .

Tychonov showed that the general product of compact spaces is compact (see the note to Exercise 15.9) so Theorem 10.1 is often referred to as Tychonov's theorem.

The same proof, or the remark that the subspace topology of a product topology is the product topology of the subspace topologies (see Exercise 15.14), gives the closely related result.

Theorem 10.2. Let $(X, \tau)$ and $(Y, \sigma)$ be topological spaces and let $\lambda$ be the product topology. If $K$ is a compact subset of $X$ and $L$ is a compact subset of $Y$, then $K \times L$ is a compact in $\lambda$.

We know (see Exercise 7.14) that the topology on $\mathbb{R}^{2}$ derived from the Euclidean metric is the same as the product topology when we give $\mathbb{R}$ the topology derived from the Euclidean metric. Theorem 9.6 thus has the following corollary.

Theorem 10.3. Let $[a, b] \times[c, d]$ with its usual (Euclidean) is compact.

The arguments of the previous section carry over to give results like the following ${ }^{7}$.

Theorem 10.4. Consider $\mathbb{R}^{2}$ with the standard (Euclidean) topology. A set $E$ is compact if and only if it is closed and bounded (that is to say, there exists a $M$ such that $\|\mathbf{x}\| \leq M$ for all $\mathbf{x} \in E$ ).

Theorem 10.5. Let $\mathbb{R}^{2}$ have the usual metric. If $K$ is a closed and bounded subset of $\mathbb{R}^{2}$ and $f: K \rightarrow \mathbb{R}$ is continuous, then $f$ is bounded and attains its bounds.

Exercise 10.6. Let $\mathbb{R}^{2}$ have the usual metric. If $K$ is a subset of $\mathbb{R}^{2}$ with the property that, whenever $f: K \rightarrow \mathbb{R}$ is continuous, then $f$ is bounded, it follows that $K$ is closed and bounded.

\footnotetext{
${ }^{7}$ Stated more poetically by Conway.

If $E$ 's closed and bounded, says Heine-Borel,

And also Euclidean, then we can tell

That, if it we smother

With a large open cover,

There's a finite refinement as well.
} 
The generalisation to $\mathbb{R}^{n}$ is left to the reader.

The next exercise brings together many of the themes of this course. The reader should observe that we know what we want the circle to look like. This exercise checks that defining the circle via quotient maps gives us what we want.

Exercise 10.7. Consider the complex plane with its usual metric. Let

$$
\partial D=\{z \in \mathbb{C}:|z|=1\}
$$

and give $\partial D$ the subspace topology $\tau$. Give $\mathbb{R}$ its usual topology and define an equivalence relation $\sim$ by $x \sim y$ if $x-y \in \mathbb{Z}$. We write $\mathbb{R} / \sim=\mathbb{T}$ and give $\mathbb{T}$ the quotient topology. The object of this exercise is to show that $\partial D$ and $\mathbb{T}$ are homeomorphic.

(i) Verify that $\sim$ is indeed an equivalence relation.

(ii) Show that, if we define $f: \mathbb{R} \rightarrow \partial D$ by $f(x)=\exp (2 \pi i x)$, then $f(U)$ is open whenever $U$ is open.

(iii) If $q: \mathbb{R} \rightarrow \mathbb{T}$ is the quotient map $q(x)=[x]$ show that $q(x)=q(y)$ if and only if $f(x)=f(y)$. Deduce that $q\left(f^{-1}(\{\exp (2 \pi i x)\})\right)=[x]$ and that the equation $F(\exp (2 \pi i x))=[x]$ gives a well defined bijection $F: \partial D \rightarrow \mathbb{T}$.

(iv) Show that $F^{-1}(V)=f\left(q^{-1}(V)\right)$ and deduce that $F$ is continuous.

(v) Show that $\mathbb{T}$ is Hausdorff and explain why $\partial D$ is compact. Deduce that $F$ is a homeomorphism.

Solution. See page 80 .

\section{Connectedness}

This section deals with a problem which the reader will meet (or has met) in her first complex variable course. Here is a similar problem that occurs on the real line. Suppose that $U$ is an open subset of $\mathbb{R}$ (in the usual topology) and $f: U \rightarrow \mathbb{R}$ is a differentiable function with $f^{\prime}(u)=0$ for all $u \in U$. We would like to conclude that $f$ is constant, but the example $U=(-2,-1) \cup(1,2)$, $f(u)=1$ if $u>0, f(u)=-1$ if $u<0$ shows that the general result is false. What extra condition should we put on $U$ to make the result true?

After some experimentation, mathematicians have come up with the following idea.

Definition 11.1. A topological space $(Y, \sigma)$ is said to be disconnected if we can find non-empty open sets $U$ and $V$ such that $U \cup V=Y$ and $U \cap V=\varnothing$. A space which is not disconnected is called connected. 
Definition 11.2. If $E$ is a subset of a topological space $(X, \tau)$ then $E$ is called connected (respectively disconnected) if the subspace topology on $E$ is connected (respectively disconnected).

The definition of a subspace topology gives the following alternative characterisation which the reader may prefer.

Lemma 11.3. If $E$ is a subset of a topological space $(X, \tau)$, then $E$ is disconnected if and only if we can find open sets $U$ and $V$ such that $U \cup V \supseteq E$, $U \cap V \cap E=\varnothing, U \cap E \neq \varnothing$ and $V \cap E \neq \varnothing$

Here is another alternative characterisation which shows that we are on the right track.

Theorem 11.4. If $E$ is a subset of a topological space $(X, \tau)$, then $E$ is disconnected if and only if we can find a non-constant continuous function $f: E \rightarrow \mathbb{R}$ which only takes the value 0 or 1 .

Proof. The proof is not hard once you see what is involved. We give the proof on page 82 .

The following deep result is now easy to prove.

Theorem 11.5. If we give $\mathbb{R}$ the usual topology, then $\mathbb{R}$ and the intervals $[a, b]$ and $(a, b)$ are connected.

Proof. See page 82.

The reader will find it instructive to use Theorem 11.4 to prove parts (i) and (iii) of the next exercise.

Exercise 11.6. (i) If $(X, \tau)$ and $(Y, \sigma)$ are topological spaces, $E$ is a connected subset of $X$ and $g: E \rightarrow Y$ is continuous, then $g(E)$ is connected. (More briefly, the continuous image of a connected set is connected.)

(ii) If $(X, \tau)$ is a connected topological space and $\sim$ is an equivalence relation on $X$, then $X / \sim$ with the quotient topology is connected.

(iii) If $(X, \tau)$ and $(Y, \sigma)$ are connected topological spaces, then $X \times Y$ with the product topology is connected.

(iv) If $(X, \tau)$ is a connected topological space and $E$ is a subset of $X$, it does not follow that $E$ with the subspace topology is connected.

Solution. See page 82.

The proof of the next example is particularly important because it gives a standard technique for using connectedness in practice. 
Example 11.7. Suppose that $E$ is a connected subset of a topological space $(X, \tau)$. Suppose that $f: E \rightarrow \mathbb{R}$ is 'locally constant' in the sense that, given any $e \in E$, we can find an open neighbourhood $U$ of e such that $f$ is constant on $U \cap E$. Then $f$ is constant.

Proof. There is a hint on page 56 and a proof on page 83 .

Exercise 11.8. Suppose that $E$ is subset of a topological space $(X, \tau)$ such that any locally constant $f: E \rightarrow \mathbb{R}$ is constant. Show that $E$ is connected.

Proof. If you need a hint, look at the proof of Theorem 11.4.

Example 11.7 and Exercise 11.8 together completely settle the question posed in the first paragraph of this section.

The following lemma outlines a very natural development.

Lemma 11.9. We work in a topological space $(X, \tau)$.

(i) Let $x_{0} \in X$. If $x_{0} \in E_{\alpha}$ and $E_{\alpha}$ is connected for all $\alpha \in A$, then $\bigcup_{\alpha \in A} E_{\alpha}$ is connected.

(ii) Write $x \sim y$ if there exists a connected set $E$ with $x, y \in E$. Then $\sim$ is an equivalence relation.

(iii) The equivalence classes $[x]$ are connected.

(iv) If $F$ is connected and $F \supseteq[x]$, then $F=[x]$.

Proof. If you need more details, see page 84 .

The sets $[x]$ are known as the connected components of $(X, \tau)$.

Connectedness is related to another, older, concept.

Definition 11.10. Let $(X, \tau)$ be a topological space. We say that $x, y \in X$ are path-connected if (when $[0,1]$ is given its standard Euclidean topology) there exists a continuous function $\gamma:[0,1] \rightarrow X$ with $\gamma(0)=x$ and $\gamma(1)=y$.

Of course, $\gamma$ is referred to as a path from $x$ to $y$.

Lemma 11.11. If $(X, \tau)$ is a topological space and we write $x \sim y$ if $x$ is path-connected to $y$, then $\sim$ is an equivalence relation.

Proof. This just a question of getting the notation under control. We give a proof on page 85 .

We say that a topological space is path-connected if every two points in the space are path-connected.

The following theorem is often useful. 
Theorem 11.12. If a topological space is path-connected, then it is connected.

Proof. This is not hard. We give a proof on page 86 .

The converse is false (see Example 11.14 below) but there is one very important case where connectedness implies path-connectedness.

Theorem 11.13. If we give $\mathbb{R}^{n}$ the usual topology, then any open set $\Omega$ which is connected is path-connected.

Proof. We give a hint on page 56 and a proof on page 86 .

The following example shows that, even in $\mathbb{R}^{2}$, we cannot remove the condition $\Omega$ open.

Example 11.14. We work in $\mathbb{R}^{2}$ with the usual topology. Let

$$
E_{1}=\{(0, y):|y| \leq 1\} \text { and } E_{2}=\{(x, \sin 1 / x): 0<x \leq 1\}
$$

and set $E=E_{1} \cup E_{2}$.

(i) Sketch E.

(ii) Explain why $E_{1}$ and $E_{2}$ are path-connected and show that $E$ is closed and connected.

(iii) Suppose, if possible, that $\mathbf{x}:[0,1] \rightarrow E$ is continuous and $\mathbf{x}(0)=$ $(1,0), \mathbf{x}(1)=(0,0)$. Explain why we can find $0<t_{1}<t_{2}<t_{3}<\ldots$ such that $x\left(t_{j}\right)=\left(\left(j+\frac{1}{2}\right) \pi\right)^{-1}$. By considering the behaviour of $t_{j}$ and $y\left(t_{j}\right)$, obtain a contradiction.

(iv) Deduce that $E$ is not path-connected.

Proof. Parts (ii) to (iv) are done on page 86.

Paths play an important role in complex analysis and algebraic topology.

\section{Compactness in metric spaces}

When we work in $\mathbb{R}$ (or, indeed, in $\mathbb{R}^{n}$ ) with the usual metric, we often use the theorem of Bolzano-Weierstrass that every sequence in a bounded closed set has a subsequence with a limit in that set. It is also easy to see that closed bounded sets are the only subsets of $\mathbb{R}^{n}$ which have the property that every sequence in the set has a subsequence with a limit in that set. This suggests a series of possible theorems some of which turn out to be false. 
Example 12.1. Give an example of metric space $(X, d)$ which is bounded (in the sense that there exists an $M$ with $d(x, y) \leq M$ for all $x, y \in X$ ) but for which there exist sequences with no convergent subsequence.

Solution. We can find such a space within our standard family of examples. See page 88 .

Fortunately we do have a very neat and useful true theorem.

Definition 12.2. A metric space $(X, d)$ is said to be sequentially compact if every sequence in $X$ has a convergent subsequence.

Theorem 12.3. A metric space is sequentially compact if and only if it is compact.

We prove the if and only if parts separately. The proof of the if part is quite simple when you see how.

Theorem 12.4. If the metric space $(X, d)$ is compact, then it is sequentially compact.

Proof. There is a hint on page 57 and a proof on page 88

The only if part is more difficult to prove (but also, in my opinion, less important). We start by proving a result of independent interest.

Lemma 12.5. Suppose that $(X, d)$ is a sequentially compact metric space and that the collection $U_{\alpha}$ with $\alpha \in A$ is an open cover of $X$. Then there exists a $\delta>0$ such that, given any $x \in X$, there exists an $\alpha(x) \in A$ such that the open ball $B(x, \delta) \subseteq U_{\alpha(x)}$.

Proof. There is a hint on page 57 and a proof on page 89.

We now prove the required result.

Theorem 12.6. If the metric space $(X, d)$ is sequentially compact, it is compact.

Proof. There is a hint on page 57 and a proof on page 89.

This gives an alternative, but less instructive, proof of the theorem of Heine-Borel.

Alternative proof of Theorem 9.5. By the Bolzano-Weierstrass theorem, $[a, b]$ is sequentially compact. Since we are in a metric space, it follows that $[a, b]$ is compact. 
If you prove a theorem on metric spaces using sequential compactness it is good practice to try and prove it directly by compactness. (See, for example, Exercise 15.19.)

The reader will hardly need to be warned that this chapter dealt only with metric spaces. Naive generalisations to general topological spaces are likely to be meaningless or false.

\section{The language of neighbourhoods}

One of the lines of thought involved in the birth of analytic topology was initiated by Riemann. We know that many complicated mathematical structures can be considered as a space which locally looks like a simpler space. Thus the surface of the globe we live on is sphere but we consider it locally as a plane (ie like $\mathbb{R}^{2}$ ). The space we live in looks locally like $\mathbb{R}^{3}$ but its global structure could be very different. For example, Riemann says 'Space would necessarily be finite if ... [we] ascribed to it a constant curvature, as long as that curvature had a positive value, however small.' [Riemann's discussion On the Hypotheses which lie at the Foundations of Geometry is translated and discussed in the second volume of Spivak's Differential Geometry.]

Unfortunately the mathematical language of his time was not broad enough to allow the expression of Riemann's insights. If we are given a particular surface such as sphere, it is easy, starting with the complete structure, to see what 'locally' and 'resembles' might mean, but, in general, we seem to be stuck in a vicious circle with 'locally' only meaningful when the global structure is known and the global structure only knowable when the meaning of 'locally' is known.

The key to the problem was found by Hilbert who, in the course of his investigations into the axiomatic foundations of geometry, produced an axiomatisation of the notion of neighbourhood in the Euclidean plane $\mathbb{R}^{2}$. By developing Hilbert's ideas, Weyl obtained what is essentially the modern definition of a Riemann surface (this object, which looks locally like $\mathbb{C}$, was another brilliant creation of Riemann).

However, although the notion of an abstract space with an abstract notion of closeness was very much in the air, there were a large number of possible candidates for such an abstraction. It was the achievement of Hausdorff to see in Hilbert's work the general notion of a neighbourhood.

Although Hausdorff defined topologies in terms of neighbourhoods, it appears to be technically easier to define topologies in terms of open sets as we have done in this course. However, topologists still use the notion of neighbourhoods. 
We have already defined an open neighbourhood of $x$ to be an open set containing $x$. We now give the more general definition.

Definition 13.1. Let $(X, \tau)$ be a topological space. If $x \in X$, we say that $N$ is a neighbourhood of $x$ if we can find $U \in \tau$ with $x \in U \subseteq N$.

The reader may check her understanding by proving the following easy lemmas.

Lemma 13.2. Let $(X, \tau)$ be a topological space. Then $U \in \tau$ if and only if, given $x \in U$, we can find a neighbourhood $N$ of $x$ with $N \subseteq U$.

Proof. The easy proof is given on page 90 .

Lemma 13.3. Let $(X, \tau)$ and $(Y, \sigma)$ be topological spaces. Then $f: X \rightarrow Y$ is continuous if and only if, given $x \in X$ and $M$ a neighbourhood of $f(x)$ in $Y$, we can find a neighbourhood $N$ of $x$ with $f(N) \subseteq M$.

Proof. The easy proof is given on page 90 .

Exercise 13.4. (i) If $(X, d)$ is a metric space, show that $N$ is a neighbourhood of $x$ if and only we can find an $\epsilon>0$ such that the open ball $B(x, \epsilon) \subseteq N$.

(ii) Consider $\mathbb{R}$ with the usual topology. Give an example of a neighbourhood which is not an open neighbourhood. Give an example of an unbounded neighbourhood. Give an example of a neighbourhood which is not connected.

Here is another related way of looking at topologies which we have not used explicitly, but which can be useful.

Definition 13.5. Let $X$ be a set. A collection $\mathcal{B}$ of subsets is called a basis if the following conditions hold.

(i) $\bigcup_{B \in \mathcal{B}} B=X$.

(ii) If $B_{1}, B_{2} \in \mathcal{B}$ and $x \in B_{1} \cup B_{2}$ we can find a $B_{3} \in \mathcal{B}$ such that $x \in B_{3} \subseteq B_{1} \cap B_{2}$

Lemma 13.6. Let $X$ be a set and $\mathcal{B}$ a collection of subsets of $X$. Let $\tau_{\mathcal{B}}$ be the collection of sets $U$ such that, whenever $x \in U$ we can find a $B \in \mathcal{B}$ such that $x \in B \subset U$.

Then $\tau_{\mathcal{B}}$ is a topology if and only if $\mathcal{B}$ is a basis.

Proof. The routine proof is given on page 91 .

Definition 13.7. If $\mathcal{B}$ is a basis and $\tau_{\mathcal{B}}$ is as in Lemma 13.6, we say that $\mathcal{B}$ is a basis $^{8}$ for $\tau_{\mathcal{B}}$.

\footnotetext{
${ }^{8}$ Sometimes $\mathcal{B}$ is called a basis of open neighbourhoods.
} 
Exercise 13.8. Consider $\mathbb{R}^{2}$ with the Euclidean norm. Show that the open discs

$$
B(॥, 1 / n)=\{\mathbf{x}:\|\mathbf{x}-\mathbf{q}\|<1 / n\}
$$

with $\mathbf{q} \in \mathbb{Q}^{2}$ and $n \geq 1, n \in \mathbb{Z}$ form a countable basis $\mathcal{B}$ for the Euclidean topology. Is it true that the intersection of two elements of $\mathcal{B}$ lies in $\mathcal{B}$ ? Give reasons.

Exercise 13.9. Let $(X, \tau)$ and $(Y, \sigma)$ be topological spaces. Show that

$$
\mathcal{B}=\{U \times V: U \in \tau, V \in \sigma\}
$$

is a basis and check, using Lemma 7.12 that it generates the product topology.

We end the course with a warning. Just as it is possible to define continuous functions in terms of neighbourhoods so it is possible to define convergence in terms of neighbourhoods. This works well in metric spaces.

Lemma 13.10. If $(X, d)$ is a metric space, then $x_{n} \rightarrow x$, if and only if given $N$ a neighbourhood of $x$, we can find an $n_{0}$ (depending on $N$ ) such that $x_{n} \in N$ for all $n \geq n_{0}$.

Proof. Immediate.

However, things are not as simple in general topological spaces.

Definition 13.11. [WARNING. Do not use this definition without reading the commentary that follows.] Let $(X, \tau)$ be a topological space. If $x_{n} \in X$ and $x \in X$ then we say $x_{n} \rightarrow x$ if, given $N$ a neighbourhood of $x$ we can find $n_{0}$ (depending on $N$ ) such that $x_{n} \in N$ for all $n \geq n_{0}$.

Any hopes that limits of sequences will behave as well in general topological spaces are dashed by the following example.

Example 13.12. Let $X=\{a, b\}$ with $a \neq b$. If we give $X$ the indiscrete topology, then, if we set $x_{n}=a$ for all $n$, we have $x_{n} \rightarrow a$ and $x_{n} \rightarrow b$.

Thus limits need not be unique.

Of course, it is possible to persist in spite of this initial shock, but the reader will find that she cannot prove the links between limits of sequences and topology that we would wish to be true. This failure is not the reader's fault. Deeper investigations into set theory reveal that sequences are inadequate tools for the study of topologies which have neighbourhood systems which are 'large in the set theoretic sense'. (Exercise 16.30 represents an attempt to show what this means.) It turns out that the deeper study of set theory reveals not only the true nature of the problem but also solutions via nets (a kind of generalised sequence) or filters (preferred by the majority of mathematicians). 


\section{Final remarks and books}

Because the notion of a topological space is so general it applies to vast collection of objects. Many useful results apply only to some subcollection and this means that the subject contains many counterexamples to show that such and such a condition is required for a certain theorem to be true.

To the generality of mankind, the longer and more complicated a piece of mathematics appears to be, the more impressive it is. Mathematicians know that the simpler a proof or a counterexample is, the easier it is to check, understand and use. Just as it is worth taking time to see if a proof can be made simpler, so it is worth taking time to see if there is a simpler counterexample for the purpose in hand.

When searching for a counterexample we may start by looking at $\mathbb{R}$ and $\mathbb{R}^{n}$ with the standard metrics and subspaces like $\mathbb{Q},[a, b],(a, b)$ and $[a, b)$. Then we might look at the discrete and indiscrete topologies on on a space. It is often worth looking at possible topologies on spaces with a small number of points (typically 3 ).

As her experience grows, the reader will have a much wider range of spaces to think about. Some like those of Exercises 15.7, 16.2, and 16.27 are very useful in their own right. Some, like that of Exercise 16.18, merely provide object lessons in how strange topologies can be,

If the reader looks at a very old book on general (or analytic) topology, she may find both the language and the contents rather different from what she is used to. In 1955, Kelley wrote a book General Topology [1] which stabilised the content and notation which might be expected in advanced course on the subject.

Texts like [3] (now in a very cheap Dover reprint ${ }^{9}$ ) and [2] (out of print) which extracted a natural elementary course quickly appeared and later texts followed the established pattern. Both [3] and [2] are short and sweet. With luck, they should be in your college library. The book of Sutherland [4] has the possible advantage of being written for a British audience and the certain advantage of being in print.

Many books on Functional Analysis, Advanced Analysis, Algebraic Topology and Differential Geometry cover the material in this course and then go on to develop it in the directions demanded by their particular subject.

${ }^{9}$ October, 2012. 


\section{References}

[1] Kelley, J. L, General Topology, Princeton N. J,. Van Nostrand, 1955. [Reissued by Springer in 1975 and Ishi Press in 2008.]

[2] Mansfield, M. J., Introduction to Topology, Princeton N. J., Van Nostrand, 1963.

[3] Mendeleson, B., Introduction to Topology, Boston Mass., Allyn and Bacon, 1962. [Now available in a Dover reprint, New York, Dover, 1990]

[4] Sutherland W. A., Introduction to Metric and Topological Spaces, Oxford, OUP, 1975.

\section{Exercises}

Exercise 15.1. Let $X$ be a set and $d: X^{2} \rightarrow \mathbb{R}$ a function with the following properties.

(i) $d(x, x)=0$ for all $x \in X$.

(ii) $d(x, y)=0$ implies $x=y$.

$(\text { iv })^{\prime} d(y, x)+d(y, z) \geq d(x, z)$ for all $x, y, z \in X$.

Show that $d$ is a metric on $X$.

Exercise 15.2. (i) If $V$ is a real inner product space and \|\| is the standard norm derived from the inner product, prove the parallelogram law

$$
\|\mathbf{a}+\mathbf{b}\|^{2}+\|\mathbf{a}-\mathbf{b}\|^{2}=2\left(\|\mathbf{a}\|^{2}+\|\mathbf{b}\|^{2}\right) .
$$

(ii) Give an example of a normed vector space where the norm cannot be derived from an inner product in a standard way.

Exercise 15.3. Let $\mathbb{R}^{N}$ have its usual (Euclidean) metric.

(i) Suppose that $f_{j}: \mathbb{R}^{n_{j}} \rightarrow \mathbb{R}^{m_{j}}$ is continuous for $1 \leq j \leq k$. Show that the map $f: \mathbb{R}^{n_{1}+n_{2}+\cdots+n_{k}} \rightarrow \mathbb{R}^{m_{1}+m_{2}+\cdots+m_{k}}$ given by

$$
f\left(\mathbf{x}_{1}, \mathbf{x}_{2}, \ldots, \mathbf{x}_{k}\right)=\left(f_{1}\left(\mathbf{x}_{1}\right), f_{2}\left(\mathbf{x}_{2}\right), \ldots, f_{k}\left(\mathbf{x}_{k}\right)\right)
$$

is continuous.

(ii) Show that the map $U: \mathbb{R}^{n} \rightarrow \mathbb{R}^{k n}$ given by

$$
U(\mathbf{x})=(\mathbf{x}, \mathbf{x}, \ldots, \mathbf{x})
$$

is continuous. 
(iii) Suppose that $g_{j}: \mathbb{R}^{n} \rightarrow \mathbb{R}^{m_{j}}$ is continuous for $1 \leq j \leq k$. Use the composition law to show that the map $g: \mathbb{R}^{n} \rightarrow \mathbb{R}^{m_{1}+m_{2}+\cdots+m_{k}}$ given by

$$
g(\mathbf{x})=\left(g_{1}(\mathbf{x}), g_{2}(\mathbf{x}), \ldots, g_{k}(\mathbf{x})\right)
$$

is continuous.

(iv) Show that the maps $A, B: \mathbb{R}^{2} \rightarrow \mathbb{R}$ given by $A(x, y)=x+y, B(x, y)=$ xy are continuous.

(v) Use the composition law repeatedly to show that the map $f: \mathbb{R}^{2} \rightarrow \mathbb{R}$ given by

$$
f(x, y)=\sin \left(\frac{x y}{x^{2}+y^{2}+1}\right)
$$

is continuous. (You may use results about maps $g: \mathbb{R} \rightarrow \mathbb{R}$

[If you have difficulty with (v), try smaller subproblems. For example, can you show that $(x, y) \mapsto x^{2}+y^{2}$ is continuous?]

Exercise 15.4. Consider $\mathbb{R}$ with the ordinary Euclidean metric.

(i) We know that $\sin : \mathbb{R} \rightarrow \mathbb{R}$ is continuous. Show that, if $U=\mathbb{R}$, then $U$ is open, but $\sin U$ is not.

(ii) We define a function $f: \mathbb{R} \rightarrow \mathbb{R}$ as follows. If $x \in \mathbb{R}$, set $\langle x\rangle=x-[x]$ and write

$$
\langle x\rangle=. x_{1} x_{2} x_{3} \ldots
$$

as a decimal, choosing the terminating form in case of ambiguity. If $x_{2 n+1}=$ 0 for all sufficiently large $n$, let $N$ be the least integer such that $x_{2 n+1}=0$ for all $n \geq N$, and set

$$
f(x)=(-1)^{N} \sum_{j=1}^{\infty} x_{2 N+2 j} 10^{N-j} .
$$

We set $f(x)=0$ otherwise.

Show that if $U$ is a non-empty open set, $f(U)=\mathbb{R}$ and so $f(U)$ is open. Show that $f$ is not continuous.

Exercise 15.5. Let $(X, d)$ be a metric space and let $r>0$. Show that

$$
\overline{B(x, r)}=\{y: d(x, y) \leq r\}
$$

is a closed set:-

(a) By using the definition of a closed set in terms of limits.

(b) By showing that the complement of $\overline{B(x, r)}$ is open.

We call $\overline{B(x, r)}$ the closed ball centre $x$ and radius $r$. 
Exercise 15.6. Prove Theorems 4.8 and 4.9 directly from the definition of a closed set in terms of limits without using open sets.

Exercise 15.7. (i) Let $(X, d)$ be a metric space. Show that

$$
\rho(x, y)=\frac{d(x, y)}{1+d(x, y)}
$$

defines a new metric on $X$.

(ii) Show that, in (i), $d$ and $\rho$ have the the same open sets.

(iii) Suppose that $d_{1}, d_{2}, \ldots$ are metrics on $X$. Show that

$$
\theta(x, y)=\sum_{n=1}^{\infty} \frac{2^{-n} d_{n}(x, y)}{1+d_{n}(x, y)}
$$

defines a metric $\theta$ on $X$.

Exercise 15.8. (This is just intended to remind of you of some elementary results on maps.) Let $X$ and $Y$ be non-empty sets and $f: X \rightarrow Y$ be a function. Suppose that $A, A^{\prime} \subseteq X, B, B^{\prime} \subseteq Y, A_{\gamma} \subseteq X, B_{\gamma} \subseteq Y$ for all $\gamma \in \Gamma$. Which of the following statements are always true and which may be false? Give a counterexample or a brief explanation in each case.

(i) $f\left(\bigcup_{\gamma \in \Gamma} A_{\gamma}\right)=\bigcup_{\gamma \in \Gamma} f\left(A_{\gamma}\right)$.

(ii) $f\left(\bigcap_{\gamma \in \Gamma} A_{\gamma}\right)=\bigcap_{\gamma \in \Gamma} f\left(A_{\gamma}\right)$.

(iii) $f\left(A \backslash A^{\prime}\right)=f(A) \backslash f\left(A^{\prime}\right)$.

(iv) $f^{-1}\left(\bigcup_{\gamma \in \Gamma} B_{\gamma}\right)=\bigcup_{\gamma \in \Gamma} f^{-1}\left(B_{\gamma}\right)$.

(v) $f^{-1}\left(\bigcap_{\gamma \in \Gamma} B_{\gamma}\right)=\bigcap_{\gamma \in \Gamma} f^{-1}\left(B_{\gamma}\right)$.

(vi) $f^{-1}\left(B \backslash B^{\prime}\right)=f^{-1}(B) \backslash f^{-1}\left(B^{\prime}\right)$.

How would your answers change if $f$ was bijective?

Exercise 15.9. (i) Suppose that $A$ is non-empty and that $\left(X_{\alpha}, \tau_{\alpha}\right)$ is a topological space. Explain what is meant by saying that $\tau$ is the smallest topology on $\prod_{\alpha \in A} X_{\alpha}$ for which each of the projection maps $\pi_{\beta}: \prod_{\alpha \in A} X_{\alpha} \rightarrow X_{\beta}$ is continuous and explain why we know that it exists. We call $\tau$ the product topology.

(ii) Show that $U \in \tau$ if and only if, given $x \in U$, we can find $U_{\alpha} \in \tau_{\alpha}$ $[\alpha \in A]$ such that

$$
x \in \prod_{\alpha \in A} U_{\alpha}
$$

and $U_{\alpha}=X_{\alpha}$ for all but finitely many of the $\alpha$.

(iii) By considering $A=[0,1]$ and taking each $\left(X_{\alpha}, \tau_{\alpha}\right)$ to be a copy of $\mathbb{R}$ show that the following condition defines a topology $\sigma$ on the space $\mathbb{R}^{[0,1]}$ of 
functions $f:[0,1] \rightarrow \mathbb{R}$. A set $U \in \sigma$ if and only if, given any $f_{0} \in U$, there exists an $\epsilon>0$ and $x_{1}, x_{2}, \ldots, x_{n} \in[0,1]$ such that

$$
\left\{f \in \mathbb{R}^{[0,1]}:\left|f\left(x_{j}\right)-f_{0}\left(x_{j}\right)\right|<\epsilon \text { for all } 1 \leq j \leq n\right\} \subseteq U .
$$

[The reader who cannot see the point of this topology is in good, but mistaken, company. The great topologist Alexandrov recalled that when Tychonov (then aged only 20) produced this definition 'His chosen ... definition seemed not only unexpected but perfectly paradoxical. [I remember] with what mistrust [I] met Tychonov's proposed definition. How was it possible that a topology induced by means of such enormous neighbourhoods, which are only distinguished from the whole space by a finite number of the coordinates, could catch any of the essential characteristics of a topological product?' However, Tychonov's choice was justified by its consequences, in particular, the generalisation (by Tychonov) of Theorem 10.1 to show that the (Tychonov) product of compact spaces is compact. This theorem called Tychonov's theorem is one of the most important in modern analysis.

In common with many of the most brilliant members of the Soviet school, Tychonov went on to work in a large number of branches of pure and applied mathematics. His best known work includes a remarkable paper on solutions of the heat equation ${ }^{10}$.]

Exercise 15.10. [The Kuratowski problem ${ }^{11}$ ] We work in a topological space $(X, \tau)$.

(i) If $A$ is a subset of $X$ show that $x \in \mathrm{Cl} A \backslash \operatorname{Int} A$ if and only if, whenever $x \in U \in \tau$, we have $U \cap A \neq \varnothing$ and $U \cap A^{c} \neq \varnothing$.

(ii) Find a set $A$ of $\mathbb{R}$ with the usual topology such that $A, \mathrm{Cl} A, \mathrm{Cl} \operatorname{Int} A$ and $\mathrm{Cl} \mathrm{Int} \mathrm{Cl} A$ are all distinct.

(iii) Show that if $A$ is any subset of $X$ then

$$
\operatorname{Int}(\mathrm{Cl}(\operatorname{Int}(\mathrm{Cl} A)))=\operatorname{Int}(\mathrm{Cl} A) .
$$

(iv) Deduce that, starting from a set $B$, the operations of taking interior and closure in various orders can produce at most seven different sets (including $A$ itself).

(v) Find a subset $B$ of $\mathbb{R}$ with the usual topology such that the operations of taking closures and interiors in various orders produce exactly seven different sets.

\footnotetext{
${ }^{10}$ A substantial part of Volume 22, Number 2 of Russian Mathematical Surveys 1967 is devoted to Tychonov and his work. The quotation from Alexandrov is taken from there.

${ }^{11}$ So called because Kuratowski solved it.
} 
Exercise 15.11. (i) Let $X=\{a, b\}$ with $a \neq b$. Show that there does not exist a largest topology contained in $\sigma=\{\varnothing,\{a\},\{b\}, X\}$. (More formally, show that there does not exist a topology $\tau$ on $X$ such that $\tau \subseteq \sigma$ and such that, if $\mu$ is any topology with $\mu \subseteq \sigma$, then $\mu \subseteq \tau$.) Compare and contrast Lemma 7.5 .

(ii) Show (with the notation of Lemma 7.15) that the quotient topology on $X / \sim$ is the largest topology (in the sense of (i)) such that $q: X \rightarrow X / \sim$ is continuous.

Exercise 15.12. Consider $\mathbb{R}$ with the usual (Euclidean) topology. Let $x \sim y$ if and only if $x-y \in \mathbb{Q}$. Show that $\sim$ is an equivalence relation. Show that $\mathbb{R} / \sim$ is uncountable but that the quotient topology on $\mathbb{R} / \sim$ is the indiscrete topology.

Exercise 15.13. (i) If $(X, \sigma)$ is a topology derived from a metric, show that, given $x \in X$, we can find open sets $U_{j}[1 \leq j]$ such that $\{x\}=\bigcap_{j=1}^{\infty} U_{j}$.

(ii) Show, by verifying the conditions for a topological space directly (so you may not quote Exercise 15.9), that the following condition defines a topology $\tau$ on the space $\mathbb{R}^{[0,1]}$ of functions $f:[0,1] \rightarrow \mathbb{R}$. A set $U \in \tau$ if and only if, given any $f_{0} \in U$, there exists an $\epsilon>0$ and $x_{1}, x_{2}, \ldots, x_{n} \in[0,1]$ such that

$$
\left\{f \in \mathbb{R}^{[0,1]}:\left|f\left(x_{j}\right)-f_{0}\left(x_{j}\right)\right|<\epsilon \text { for } 1 \leq j \leq n\right\} \subseteq U .
$$

(iii) Show that the topology $\tau$ is Hausdorff but cannot be derived from a metric.

Exercise 15.14. Let $(X, \tau)$ and $(Y, \sigma)$ be topological spaces with subsets $E$ and $F$. Let the subspace topology on $E$ be $\tau_{E}$ and the subspace topology on $F$ be $\sigma_{F}$. Let the product topology on $X \times Y$ derived from $\tau$ and $\sigma$ be $\lambda$ and let the product topology on $E \times F$ derived from $\tau_{E}$ and $\sigma_{F}$ be $\mu$. Show that $\mu$ is the subspace topology on $E \times F$ derived from $\lambda$.

Exercise 15.15. (i) Let $\mathcal{H}_{i}$ be a collection of subsets of $X_{i}$ and let $\tau_{i}$ be the smallest topology on $X_{i}$ containing $\mathcal{H}_{i}[i=1,2]$. If $f: X_{1} \rightarrow X_{2}$ has the property that $f^{-1}(H) \in \mathcal{H}_{1}$ whenever $H \in \mathcal{H}_{2}$, show that $f$ is continuous (with respect to the topologies $\tau_{1}$ and $\tau_{2}$ ).

(ii) Suppose that $(X, \tau)$ and $(Y, \sigma)$ are topological space and we give $X \times Y$ the product topology. If $(Z, \lambda)$ is a topological space, show that $f: Z \rightarrow X \times Y$ is continuous if and only if $\pi_{X} \circ f: Z \rightarrow X$ and $\pi_{Y} \circ f: Z \rightarrow Y$ are continuous. 
(iii) Let $\mathbb{R}$ have the usual topology (induced by the Euclidean metric) and let $\mathbb{R}^{2}$ have the product topology (which we know to be the usual topology induced by the Euclidean metric). Define

$$
f(x, y)= \begin{cases}\frac{x y}{x^{2}+y^{2}} & \text { if }(x, y) \neq(0,0), \\ 0 & \text { if }(x, y)=(0,0) .\end{cases}
$$

Show that, if we define $h_{x}(y)=g_{y}(x)=f(x, y)$ for all $(x, y) \in \mathbb{R}^{2}$, then the function $h_{x}: \mathbb{R} \rightarrow \mathbb{R}$ is continuous for each $x \in \mathbb{R}$ and the function $g_{y}: \mathbb{R} \rightarrow \mathbb{R}$ is continuous for each $y \in \mathbb{R}$. Show, however, that $f$ is not continuous.

Exercise 15.16. In complex variable theory we encounter 'uniform convergence on compacta'. This question illustrates the basic idea in the case of $C(\Omega)$ the space of continuous functions $f: \Omega \rightarrow \mathbb{C}$ where

$$
\Omega=\{z \in \mathbb{C}:|z|<1\} .
$$

(i) Show, by means of an example, that an $f \in C(\Omega)$ need not be bounded on $\Omega$.

(ii) Explain why

$$
d_{n}(f, g)=\sup _{|z| \leq 1-1 / n}|f(z)-g(z)|
$$

exists and is finite for each $n \geq 1$ and all $f, g \in C(\Omega)$. Show that $d_{n}$ satisfies the triangle law and symmetry but give an example of a pair of functions $f, g \in C(\Omega)$ with $f \neq g$ yet $d_{n}(f, g)=0$.

(iii) Show that

$$
d(f, g)=\sum_{n=1}^{\infty} \frac{2^{-n} d_{n}(f, g)}{1+d_{n}(f, g)}
$$

exists and is finite for all $f, g \in C(\Omega)$.

(iv) Show that d is a metric on $C(\Omega)$. [If you require a hint, do Exercise 15.7 (i).]

Exercise 15.17. (i) Show that the closure of a connected set is connected.

(ii) Deduce that connected components are closed.

(iii) Show that if there are only finitely many components they must all be open.

(iv) Find the connected components of

$$
\{0\} \cup \bigcup\{1 / n: n \geq 1, n \in \mathbb{Z}\}
$$


with the usual metric.

Which are open in the subspace topology and which are not? Give reasons.

(v) Is it true that the interior of a connected set is always connected? Give a proof or a counterexample.

Exercise 15.18. (i) If $(X, \tau)$ and $(Y, \sigma)$ are topological spaces, $E$ is a pathconnected subset of $X$ and $g: E \rightarrow Y$ is continuous, show that $g(E)$ is path-connected. (More briefly, the continuous image of a path-connected set is path-connected.)

(ii) If $(X, \tau)$ is a path-connected topological space and $\sim$ is an equivalence relation on $X$, show that $X / \sim$ with the quotient topology is path-connected.

(iii) If $(X, \tau)$ and $(Y, \sigma)$ are path-connected topological spaces, show that $X \times Y$ with the product topology is path-connected.

(iv) If $(X, \tau)$ is a path-connected topological space and $E$ is a subset of $X$, show that it does not follow that $E$ with the subspace topology is pathconnected.

Exercise 15.19. Suppose that $(X, d)$ is a compact metric space, $(Y, \rho)$ is a metric space and $f: X \rightarrow Y$ is continuous. Explain why, given $\epsilon>0$, we can find, for each $x \in X, a \delta_{x}>0$ such that, if $d(x, y)<2 \delta_{x}$, it follows that $\rho(f(x), f(y))<\epsilon$. By considering the open cover $B\left(x, \delta_{x}\right)$ and using compactness, show that there exists a $\delta>0$ such that $d(x, y)<\delta$ implies $\rho(f(x), f(y))<\epsilon$. (In other words, a continuous function from a compact metric space to a metric space is uniformly continuous.)

Exercise 15.20. Which of the following spaces are homeomorphic and which are not? Give reasons.

(i) $\mathbb{R}$ with the usual topology.

(ii) $\mathbb{R}$ with the discrete topology.

(iii) $\mathbb{Z}$ with the discrete topology.

(iv) $[0,1]$ with the usual topology.

(v) $(0,1)$ with the usual topology.

[This is rather feeble question but in this short course we have not found enough topological properties to distinguish between some clearly distinguishable topological spaces. We return to this matter in Exercise 15.21.]

Exercise 15.21. Suppose that $f:[0,1] \rightarrow \mathbb{R}$ and $g:[0,1] \rightarrow \mathbb{R}$ are continuous maps with $f(0)=-1, f(1)=2, g(0)=0$ and $g(1)=1$. Show that

$$
f([0,1]) \cap g([0,1]) \neq \varnothing
$$

(In other words, the two paths must cross.) 
Show that $\mathbb{R}$ and $\mathbb{R}^{2}$ with the usual topologies are not homeomorphic. Are $[0,1]$ and the circle

$$
\{z \in \mathbb{C}:|z|=1\}
$$

homeomorphic and why?

(But are $\mathbb{R}^{2}$ and $\mathbb{R}^{3}$ homeomorphic? Questions like this form the beginning of modern algebraic topology.)

Exercise 15.22. Which of the following statements are true and which false. Give a proof or counter-example.

(i) If a topological space $(X, \tau)$ is connected then the only sets which are both open and closed are $X$ and $\varnothing$.

(ii) If every set in a topological space $(X, \tau)$ is open or closed (or both) then $\tau$ is the discrete topology.

(iii) Every open cover of $\mathbb{R}$ with the usual topology has a countable subcover.

(iv) Suppose that $\tau$ and $\sigma$ are topologies on a space $X$ with $\sigma \supseteq \tau$. Then, if $(X, \tau)$ is connected, so is $(X, \sigma)$.

$(v)$ Suppose that $\tau$ and $\sigma$ are topologies on a space $X$ with $\sigma \supseteq \tau$. Then, if $(X, \sigma)$ is connected, so is $(X, \tau)$.

Exercise 15.23. [Bases of neighbourhoods.] (i) Let $(X, \tau)$ be a topological space. Write $\mathcal{N}_{x}$ for the set of neighbourhoods of $x \in X$. Prove the following results.

(1) $\mathcal{N}_{x} \neq \varnothing$.

(2) If $N \in \mathcal{N}_{x}$, then $x \in N$.

(3) If $N, M \in \mathcal{N}_{x}$, then $N \cap M \in \mathcal{N}_{x}$.

(4) If $N \in \mathcal{N}_{x}$ and $M \supseteq N$, then $M \in \mathcal{N}_{x}$.

(5) If $N \in \mathcal{N}_{x}$ then there exists an $U \in \mathcal{N}_{x}$ such that $U \subseteq N$ and $U \in \mathcal{N}_{y}$ for all $y \in U$.

(ii) Suppose that $X$ is a set such that each $x \in X$ is associated with a collection $\mathcal{N}_{x}$ of subsets of $X$. If conditions (1) to (4) of part (ii) hold, show that the family $\tau$ of sets $U$ such that, if $x \in U$, then we can find an $N \in \mathcal{N}_{x}$ with $N \subseteq U$ is a topology on $X$. If, in addition, condition (5) holds show that $\mathcal{N}_{x}$ is a collection of $\tau$-neighbourhoods of $x$ for each $x \in X$.

Exercise 15.24. Consider $\mathbb{R}^{2}$ with the usual Euclidean topology. Let

$$
E=\{(x,-1): x \in \mathbb{R}\} \cup\{(x, 1): x \in \mathbb{R}\}
$$

and give E the subspace topology. 
Define a relation $\sim$ on $E$ by taking

$$
\begin{aligned}
(x, y) \sim(x, y) & \text { for all }(x, y) \in E \\
(x, y) \sim(x,-y) & \text { for all }(x, y) \in E \text { with } x \neq 0 .
\end{aligned}
$$

Show that that $\sim$ is an equivalence relation on $E$.

Now give $E / \sim$ the equivalence relation. Show that if $[(x, y)] \in E / \sim$ we can find an open neighbourhood $U$ of $[(x, y)]$ which is homeomorphic to $\mathbb{R}$. Show, however, that $E / \sim$ is not Hausdorff.

[This nasty example shows that 'looks nice locally' is not sufficient to give 'looks nice globally'. It is good start to a course in differential geometry to ask what extra conditions are required to make sure that a space that 'looks locally like a line' 'looks globally like a line or a circle'.]

\section{More exercises}

There is an ancient superstition in Cambridge that 12 exercises are necessary and sufficient to learn six hours of lectures. If the reader does not share this superstition she may find the following exercises useful.

Exercise 16.1. Suppose that $p$ is a prime. If $m, n \in \mathbb{Z}$ we set $d(m, n)=0$ if $m \neq 0$ and, otherwise, $d(m, n)=\frac{1}{r}$ where $p^{r}$ divides $m-n$ but $p^{r+1}$ does not. Show that $d$ is a metric on $\mathbb{Z}$. Show that the sequence 2013, 20013, 200013, ... tends to a limit in this metric.

Show that the sequence $5^{n}+5^{n-1}+\ldots+5+1$ is Cauchy but does not converge.

Exercise 16.2. Let $q \geq 1$. Let $l^{q}$ be the set of sequences of real numbers $\mathbf{a}=$ $\left(a_{1}, a_{2}, \ldots\right)$ with $\sum_{j=1}^{\infty}\left|a_{j}\right|^{q}$ convergent. We write $\|\mathbf{a}\|_{q}=\left(\sum_{j=1}^{\infty}\left|a_{j}\right|^{q}\right)^{1 / q} .$.

(i) If $\mathbf{a}$ is a sequence and we write

$$
\mathbf{a}[N]=\left(a_{1}, a_{2}, \ldots, a_{N}, 0,0, \ldots\right)
$$

show that $\mathbf{a} \in l^{q}$ if and only if $\|\mathbf{a}[N]\|_{q}$ is bounded and that, if $\mathbf{a} \in l^{q}$, then

$$
\|\mathbf{a}[N]\|_{q} \rightarrow\|\mathbf{a}\|_{q}
$$

as $N \rightarrow \infty$.

(ii) Show, using (i), that $l^{1}$ and $l^{2}$ are real vector spaces, that \|\|$_{1}$ is a norm on $l^{1}$ and that \|\|$_{2}$ is a norm on $l^{2}$.

(iii) Show that $l^{2} \supseteq l^{1}$.. 
(iv) Show that the identity map

$$
\iota:\left(l^{1},\|\|_{2}\right) \rightarrow\left(l^{1},\|\|_{1}\right)
$$

(that is to say from $l^{1}$ with the subspace norm derived from \|\|$_{2}$ to $l^{1}$ with the norm \|\|$_{2}$ ) is not continuous.

Show that the identity map

$$
\iota:\left(l^{1},\|\|_{1}\right) \rightarrow\left(l^{1},\|\|_{2}\right)
$$

is continuous.

(v) If $f\left(a_{1}, a_{2}, \ldots\right)=\left(a_{1}, a_{2} / 2, a_{3} / 3, \ldots\right)$ show that

Show that

$$
f\left(l^{1},\|\|_{1}\right) \rightarrow\left(l^{1},\|\|_{2}\right)
$$

is well defined and continuous.

Exercise 16.3. Suppose that $d_{1}$ and $d_{2}$ are metrics on a space $X$. Show that it is a sufficient condition for them to generate the same topology that there exists a $K \geq 1$ with

$$
K d_{1}(x, y) \geq d_{2}(x, y) \geq K^{-1} d_{1}(x, y)
$$

for all $x, y \in X$

By considering d defined by $d(x, y)=|x-y|^{1 / 2}$ for $x, y \in \mathbb{R}$, or otherwise, show that the condition is not necessary.

Show, however, that, if \|\|$_{A}$ and \|\|$_{B}$ are norms on a vector space $V$, then it is a necessary and sufficient condition for them to generate the same topology that there exists a $K \geq 1$ with

$$
K\|\mathbf{x}\|_{A} \geq\|\mathbf{x}\|_{B} \geq K^{-1}\|\mathbf{x}\|_{A}
$$

for all $\mathrm{x} \in V$.

Exercise 16.4. There are many proofs that there exist an infinity of primes. Here is a remarkable one published by Fürstenberg in 1955 when he was still an undergraduate.

Consider $\mathbb{Z}$. Let $\mathcal{A}$ be the collection of arithmetic progressions

$$
\{a n+b: n \in \mathbb{Z}\}
$$

with $a \neq 0$ and let $\tau$ be the collection of unions of sets in $\mathcal{A}$ together with $\mathbb{Z}$.

(i) Show that $\tau$ is a topology.

(ii) Show that every $A \in \mathcal{A}$ is closed in the topology $\tau$. 
(iii If $\mathcal{A}^{*}$ consists of the arithmetic progressions

$$
\{a p: n \in \mathbb{Z}\}
$$

with $p$ prime, identify

$$
\mathbb{Z} \backslash \bigcup_{A \in \mathcal{A}^{*}} A
$$

(iv) Suppose, if possible, there are only finitely many primes. Use parts (ii) and (iii) to obtain a contradiction.

(v) Applaud.

Exercise 16.5. Show by means of an example that the following statement may be false.

If $E$ is subset of $\mathbb{R}$, with the usual topology, then there exists a unique open set $V$ such that

(a) $V \supseteq E$,

(b) if $U$ is a open set with $U \supseteq E$, then $U \supseteq V$.

Exercise 16.6. Show that the following statements about a topological space $(Y, \sigma)$ are equivalent.

(i) $(Y, \sigma)$ is Hausdorff.

(ii) If $Y \times Y$ is given the product topology, the diagonal

$$
\{\Delta=\{(y, y): y \in Y\}
$$

is closed.

(iii) For any topological space $(X, \tau)$ and any continuous functions $f, g$ : $X \rightarrow Y$, the set

$$
\{x \in X: f(x)=g(x)\}
$$

is closed.

Use the equivalence of (i) and (iii) to produce an alternative proof of the result of Exercise 8.7.

Exercise 16.7. We work on $\mathbb{R}$. Let $\tau_{1}$ be the collection of sets which are unions of half open intervals $[a, b)$ (including $\varnothing$ ). Let $\tau_{2}$ be the collection of subsets of $E$ such that either $E=\varnothing$ or $\mathbb{R} \backslash E$ is finite.

(i) Show that $\tau_{1}$ and $\tau_{2}$ are topologies.

(ii) Is $\tau_{1}$ Hausdorff?

(iii) Is $\tau_{2}$ Hausdorff?

(iv) Is $\tau_{1}$ compact?

(v) Is $\tau_{2}$ compact?

(vi) Is $\tau_{1}$ connected? 
(vii) Is $\tau_{2}$ connected?

(viii) Is the identity map $\iota:\left(\mathbb{R}, \tau_{1}\right) \rightarrow\left(\mathbb{R}, \tau_{2}\right)$ continuous?

(ix) Is the identity map $\iota:\left(\mathbb{R}, \tau_{2}\right) \rightarrow\left(\mathbb{R}, \tau_{1}\right)$ continuous?

Exercise 16.8. Let $\left(X, \tau_{X}\right),\left(Y, \tau_{Y}\right),\left(Z, \tau_{Z}\right)$ be topological spaces. Suppose that we give $X \times Y$ the product topology $\tau_{X \times Y}$ derived from $\tau_{X}$ and $\tau_{Y},(X \times$ $Y) \times Z$ the product topology $\tau_{X \times Y}$ derived from $\tau_{X \times Y}$ and $\tau_{Z}$ and so on.

(i) Show that $\left(X \times Y, \tau_{X \times Y}\right)$ and $\left(Y \times X, \tau_{Y \times X}\right)$ are homeomorphic.

(ii) Show that $\left((X \times Y) \times Z, \tau_{(X \times Y) \times Z)}\right.$ and $\left(X \times(Y \times Z), \tau_{X \times(Y \times Z)}\right)$ are homeomorphic.

Exercise 16.9. Does there exist an infinite compact subset of the rationals (with the usual metric)? Give reasons.

\section{Exercise 16.10. [The finite intersection property]}

(i) (This result is almost trivial but very useful.) Show that a topological space $(X, \tau)$ is compact if and only if it has the following property.

If $\mathcal{F}$ is a collection of closed sets with the 'finite intersection property'

$$
F_{1}, F_{2}, \ldots, F_{n} \in \mathcal{F} \Rightarrow \bigcap_{j=1}^{n} F_{j} \neq \varnothing,
$$

then

$$
\bigcap_{F \in \mathcal{F}} F \neq \varnothing
$$

(ii) We work in $\mathbb{R}$ with the usual metric. Give an example of of sequence of non-empty bounded open sets $O_{j}$ such that

$$
O_{1} \supseteq O_{2} \supseteq O_{3} \supseteq \ldots \text {, but } \bigcap_{j=1}^{\infty} O_{j}=\varnothing \text {. }
$$

Give an example of of sequence of non-empty closed sets $F_{j}$ such that

$$
F_{1} \supseteq F_{2} \supseteq F_{3} \supseteq \ldots, \text { but } \bigcap_{j=1}^{\infty} F_{j}=\varnothing .
$$

Exercise 16.11. Consider the space $C_{\mathbb{R}}([0,1])$ of continuous functions $f$ : $[0,1] \rightarrow \mathbb{R}$ (with the usual metrics). Show that

$$
\|f\|_{\infty}=\sup _{t \in[0,1]}|f(t)|
$$


is a well defined norm on $C_{\mathbb{R}}([0,1])$. The general principle of uniform convergence which you meet in Analysis II tells you that this norm is complete.

Show that

$$
B=\left\{f:\|f\|_{\infty} \leq 1\right\}
$$

is closed and bounded but not compact.

[Contrast the theorem of Heine-Borel.]

Exercise 16.12. (Traditional) Fairyland may be considered as a perfectly flat, endless plane. Good Queen Ermentrude has planted an infinite forest of trees in such a way that, wherever she looks from her throne, she sees a tree. A troop of renegade beavers decide to gnaw down all but a finite set of trees without being seen by the Queen. Can they always do this? (Queen Ermentrude is open minded and only plants open trees.)

Exercise 16.13. [The one point compactification] Let $(X$, tau $)$ be a topological space (which may or may not be compact).

(i) Write $X^{*}=X \cup\{\infty\}$. (Note that $\infty$ is just an object which is not in $X$. We could follow Hilbert and take it to be 'beer mug'.)

Let $\tau^{*}$ be the collection of sets $E \subseteq X^{*}$ such that either $E \in \tau$ or $E=$ $(X \backslash K) \cup\{\infty\}$, where $K$ is a closed compact subset of $X$. Show that $\tau^{*}$ is topology on $X^{*}$ and $\left(X^{*}, \tau^{*}\right)$ is compact. Show that the subspace topology $\tau_{X}^{*}$ induced on $X$ by $\tau^{*}$ is $\tau$.

(ii) (A well known variation.) Let $\tilde{\mathbb{R}}=\mathbb{R} \cup\{-\infty, \infty\}$ (so, this time, we add a beer mug and a sherry glass).

Show that the collection $\mathcal{E}$ of sets of the form

$$
[-\infty, a)=-\infty \cup\{x \in \mathbb{R}: x<a\},(b, \infty]=-\infty \cup\{x \in \mathbb{R}: b<x\}
$$

together with the open intervals $(a, b)$ form the basis for a compact topology $\tilde{\tau}$ on $\tilde{\mathbb{R}}$.

Exercise 16.14. Let $P_{1}, P_{2}, \ldots, P_{n}$ be distinct points in $\mathbb{R}^{2}$ and $A=$ $\left\{P_{1}, P_{2}, \ldots, P_{n}\right\}$. Let $\mathbf{x} \sim \mathbf{y}$ if and only if $\mathbf{x}=\mathbf{y}$ or $\mathbf{x}, \mathbf{y} \in A$. Show that $\sim$ is an equivalence relation on $\mathbb{R}^{2}$.

If $\tau$ is the usual Euclidean topology on $\mathbb{R}^{2}$, show that the topology $\tau / \sim$ on $\mathbb{R}^{2} / \sim$ can be derived from a metric.

[Hint: The metric usually chosen is called the London underground metric.]

Exercise 16.15. Let $X$ and $Y$ be non-empty topological spaces, and give $X \times Y$ the product topology. Show that

$$
(x, y) \sim(u, v) \Leftrightarrow y=v
$$

is an equivalence relation on $X \times Y$ Show that, as one might hope, the space $X \times Y / \sim$ with the quotient topology is homeomorphic to $Y$. 
Exercise 16.16. If $(X, d)$ is a metric space, $x \in X$ and $E$ is non-empty subset of $X$, we set

$$
f_{E}(x)=d(x, E)=\inf \{d(x, e): e \in E\} .
$$

(i) Show that the map $f_{E}$ from $(X, d)$ to $\mathbb{R}$ with its usual metric is continuous.

(ii) Show that $E$ is closed if and only if $d(x, E)>0$ for all $x \notin E$.

(iii) By using the functions $f_{E_{1}}$ and $f_{E_{2}}$, or otherwise, show that, if $E_{1}$ and $E_{2}$ are disjoint closed subsets of $X$ then there exists a continuous function $f: X \rightarrow \mathbb{R}$ with the properties that $1 \geq f(x) \geq 0$ for all $x \in X$ and

$$
f(x)= \begin{cases}1 & \text { if } x \in E_{1}, \\ 0 & \text { if } x \in E_{2} .\end{cases}
$$

Deduce that we can find disjoint open sets $U_{1}$ and $U_{2}$ such that $U_{1} \supseteq E_{1}$ and $U_{2} \supseteq E_{2}$.

Exercise 16.17. (This continues on from parts (i) and (ii) of Exercise 16.16.)

(i) We work in a metric space $(X, d)$. Consider two non-empty disjoint sets $E$ and $G$. If $E$ is compact and $G$ is closed, show that there exists a $\delta>0$ such that

$$
d(e, g) \geq \delta
$$

for all $e \in E$ and $g \in G$.

(ii) Find two non-empty disjoint closed sets $E$ and $G$ in $\mathbb{R}$ with the usual metric such that

$$
\inf _{e \in E, g \in G}|e-g|=0 .
$$

Exercise 16.18. We work on $\mathbb{R}$. Let $\tau$ consist of all sets of the form $U \cup S$ where $U$ is an open set for the usual Euclidean topology and $S$ is a subset of the irrationals.

(i) Show that $\tau$ is a topology. (It is called the 'scattered topology'.)

(ii) Show that $\tau$ is Hausdorff.

(iii) Show that $\{x\}$ is open if and only if $x$ is irrational.

Exercise 16.19. Let $(X, \tau)$ be a topological space and $E$ and $F$ subsets with the subspace topologies $\tau_{E}, \tau_{F}$. Suppose that $E \cup F=X$, that $(Y, \sigma)$ is another topological space and $g: X \rightarrow Y$ a function. Suppose that $\left.g\right|_{E}:\left(E, \tau_{E}\right) \rightarrow$ $(Y, \sigma)$ and $\left.g\right|_{F}:\left(F, \tau_{F}\right) \rightarrow(Y, \sigma)$ are continuous.

Which of the following statements are always true and which may be false? Give proofs or counterexamples.

(i) If $E$ and $F$ are open, then $g:(X, \tau) \rightarrow(Y, \sigma)$ is continuous.

(ii) If $E$ and $F$ are closed, then $g:(X, \tau) \rightarrow(Y, \sigma)$ is continuous.

(iii) If $E$ is open and $F=X \backslash E$, then $g:(X, \tau) \rightarrow(Y, \sigma)$ is continuous. 
Exercise 16.20. (Requires the idea of uniform convergence from Analysis II.) This example of a space filling curve due to Liu Wen is simple rather than pretty.

Let $\delta_{k}=[k / 10,(k+1) / 10]$ for $0 \leq k \leq 9$. Let $f, g:[0,1] \rightarrow \mathbb{R}$ satisfy the following conditions:-

$$
f(t)=\left\{\begin{array}{ll}
0 & \text { when } t \in \delta_{1} \cup \delta_{3} \\
1 & \text { when } t \in \delta_{5} \cup \delta_{7}
\end{array} \quad g(t)= \begin{cases}0 & \text { when } t \in \delta_{1} \cup \delta_{5} \\
1 & \text { when } t \in \delta_{3} \cup \delta_{7}\end{cases}\right.
$$

and $f(0)=f(1)=0, g(0)=g(1)=0$. Sketch such a function.

Set $F(t+n)=f(t), G(t+n)=g(t)$ for all $t \in[0,1]$ and $n \in \mathbb{Z}$. Explain why, if we set

$$
\left.\left.\phi(t)=\sum_{k=1}^{\infty} 2^{-k} F\left(10^{k-1}\right) t\right), \psi(t)=\sum_{k=1}^{\infty} 2^{-k} G\left(10^{k-1}\right) t\right),
$$

the map $t \mapsto\left(\phi(t), \psi(t)\right.$ is a continuous map of $[0,1]$ to $[0,1]^{2}$ (with the usual metrics).

If

$$
x=\sum_{j=1}^{\infty} x_{j} 2^{-j} \text { and } y=\sum_{j=1}^{\infty} y_{j} 2^{-j}
$$

with $x_{j}, y_{j} \in\{0,1\}$, find $t_{j} \in\{1,3,5,7\}$ such that, writing

$$
t=\sum_{j=1}^{\infty} t_{j} 10^{-j}
$$

we have $(\phi(t), \psi(t))=(x, y)$.

Conclude that there is a continuous surjective map from $[0,1]$ to $[0,1]^{2}$.

Exercise 16.21. We use the standard Euclidean metrics. Show that there does not exist a continuous injection $f:[0,1]^{2} \rightarrow[0,1]$.

[Hint: Let $E=[0,1]^{2} \backslash\{\mathbf{a}\}$ for some fixed $\mathbf{a}$ and consider $\left.\left.f\right|_{E}.\right]$

Exercise 16.22. We use the standard metric. Show that there does not exist a continuous function $f: \mathbb{R} \rightarrow \mathbb{R}$ such that

$$
x \in \mathbb{Q} \Leftrightarrow f(x) \notin \mathbb{Q} .
$$

Does there exist a continuous function $g: \mathbb{R} \rightarrow \mathbb{R}$ such that

$$
x \in \mathbb{Q} \Leftrightarrow g(x) \in \mathbb{Q} ?
$$

Give reasons for your answer. 
Exercise 16.23. Which, if any, of the following subsets of $\mathbb{R}^{2}$ with the usual topology are connected?

(i) $A=\{(x, y): x \in \mathbb{Q}\}$.

(ii) $A=\{(x, y): x \in \mathbb{Q}\} \cup\{(x, y): y \in \mathbb{Q}\}$.

Exercise 16.24. Consider a compact metric space $(X, d)$. Show that there exists a $K$ such that $d(x, y) \leq K$ for all $x, y \in X$. If $E$ is a non-empty subset of of $X$, we define the diameter $\Delta(E)$ of $E$ by

$$
\Delta(E)=\sup _{(x, y) \in E} d(x, y)
$$

Show that if $\left\{U_{\lambda}\right\}_{\lambda \in \Lambda}$ is an open cover of $X$, then there exists a $\delta>0$ such that every non-empty subset $E$ with $\Delta E<\delta$ lies in some $U_{\lambda}$.

Exercise 16.25. We work in a metric space $X, d)$. Suppose that $E_{1}, E_{2}$, ... are connected sets with $E_{1} \supseteq E_{2} \supseteq \ldots$. Show that, if the $E_{j}$ are compact, $\bigcap_{j=1}^{\infty} E_{j}$ is connected.

[Hint: You may find Exercise 16.16 (iii) useful.]

Give an example in $\mathbb{R}^{2}$ with the usual Euclidean topology to show that the result may fail if we replace 'compact' by 'closed'.

Exercise 16.26. In this question you may quote the result that the product of two compact spaces is compact, but no other result on product topologies.

Suppose that $(X, \tau),(Y, \sigma)$ are topological spaces and we give $X \times Y$ the product topology $\rho$.

(i) Show that, if $x \in X$, then

$$
\{\{y \in Y:(x, y) \in U\}: U \in \rho\}=\sigma .
$$

(ii) Give an example with $X$ and $Y$ each consisting of 2 points of a topology $\eta$ on $X \times Y$ such that

$$
\{\{x \in X:(x, y) \in U\}: U \in \eta\}=\sigma
$$

for each $y \in Y$ and

$$
\{\{y \in Y:(x, y) \in U\}: U \in \eta\}=\tau
$$

for each $x \in X$, but $\eta \neq \rho$.

(iii) Prove the following results.

(a) $\rho$ Hausdorff $\Leftrightarrow \tau, \sigma$ Hausdorff.

(b) $\rho$ compact $\Leftrightarrow \tau, \sigma$ compact.

(c) $\rho$ connected $\Leftrightarrow \tau, \sigma$ connected.

(d) $\rho$ path-connected $\Leftrightarrow \tau, \sigma$ path-connected. 
Exercise 16.27. Consider the space of sequences of zeros and ones $X=$ $\{0,1\}^{\mathbb{N}}$. Let us set

$$
d(\mathbf{x}, \mathbf{y})=2^{-n}
$$

if $x_{j}=y_{j}$ for $1 \leq j \leq n-1, x_{n} \neq y_{n}$ and $d(\mathbf{x}, \mathbf{x})=0$.

(i) Show that d is a metric.

(ii) Show that $(X, d)$ is complete.

(iii) Show that $(X, d)$ is compact.

(iv) Show that no point in $(X, d)$ is isolated (that is to say, no one point set $\{\mathbf{x}\}$ is open).

(v) Show that the connected components of $(X, d)$ are the one point sets.

(vi) Show that $X \times X$ with the product topology is homeomorphic to $X$. [The space just described may look nasty at first sight, but is, in fact, both elegant and useful.]

Exercise 16.28. (i) Consider a topological space $(X, \tau)$. If $X$ has a countable dense subset show that so does every subset of $X$ (for the subspace topology).

(ii) Consider a metric space $(Y, \rho)$. If $Y$ has a countable dense subset show that the associated topology has a countable basis.

Exercise 16.29. Show that the collection of half open intervals $[a, b)$ form a basis. Consider the 'half open topology' $\tau_{H}$ on $\mathbb{R}$ is generated by this basis.

(i) Show that $\tau_{H}$ is Hausdorff $\{x\}$.

(ii) Show that the connected components of $\left(\mathbb{R}, \tau_{H}\right)$ are the one point sets

(iii) Show that $[a, b]$ with $a<b$ is not compact in $\tau_{H}$.

(vi) Consider $\mathbb{R}^{2}$ with the product topology $\sigma_{H}$ obtained from $\tau_{H}$. Show that $\mathbb{R} \times \mathbb{R}$ has a countable dense subset.

(v) Show that the subspace topology on $Z=\{(x, x): x \in \mathbb{R}\}$ derived from $\sigma_{H}$ is discrete.

(vii) Use Exercise 16.28 to show that $\sigma_{H}$ is not derived from a metric. Deduce that $\tau_{H}$ is not derived from a metric.

Exercise 16.30. Consider the collection $X_{*}$ of all functions $f:[0,1] \rightarrow \mathbb{R}$ with $f(x)>0$ for $x>0, f(0)=0$ and $f(x) \rightarrow 0$ as $x \rightarrow 0$. We take $X=X_{*} \cup\left\{f_{0}\right\}$ where $f_{0}$ is the zero function defined by $f_{0}(x)=0$ for all $x \in[0,1]$. If $g \in X_{*}$, write

$$
U_{g}=\{f \in X: f(x) / g(x) \rightarrow 0 \text { as } x \rightarrow 0\} .
$$

Show that, given $g_{1}, g_{2} \in X_{*}$ we can find a $g_{3} \in X_{*}$ such that

$$
U_{g_{3}} \subseteq U_{g_{1}} \cap U_{g_{2}}
$$


Conclude that, if $\tau$ consists of $\varnothing$ together with all those sets $V$ such that $V \supseteq U_{g}$ for some $g \in X_{*}$, then $\tau$ is a topology on $X$. Show that

$$
\bigcap_{g \in X_{*}} U_{g}=\left\{f_{0}\right\}
$$

Now suppose $g_{j} \in X^{*}$. If we set $g(0)=0$ and

$$
g(t)=n^{-1} \min _{1 \leq j \leq n} g_{j}(t) \text { for } t \in\left((n+1)^{-1}, n^{-1}\right],
$$

show that $g \in X^{*}$ and $g_{j} \notin U_{g}$. Conclude that, although every open neighbourhood of $f_{0}$ contains infinitely many points and the intersection of the open neighbourhoods of $f_{0}$ is the one point set $\left\{f_{0}\right\}$, there is no sequence $g_{j}$ with $g_{j} \neq f_{0}$ such that $g_{j} \rightarrow f_{0}$.

If you just accept this result without thought, it is not worth doing the question. You should compare and contrast the metric case. I would say that $f_{0}$ is 'surrounded by too many neighbourhood-shells to be approached by a sequence', but the language of the course is inadequate to make this thought precise.

I am told that the ancient Greek geometers used a similar counterexample for a related purpose.]

Exercise 16.31. (i) Show that the following two statements about a metric space $(X, d)$ are equivalent.

(A) There is a complete metric $\rho$ on $X$ which induces the same topology as $d$. to $(X, d)$.

(B) There is a complete metric space $(Y, \theta)$ which is homeomorphic

(ii) Consider $\mathbb{Q}$ with the usual metric $d$ and a metric $\rho$ which induces the same topology as $d$. Write $\mathbb{Q}=\left\{q_{1}, q_{2}, \ldots\right\}$. Let $y_{0}=0, r_{0}=1$. Show that we can find inductively $y_{n} \in \mathbb{Q}$ and $r_{n}>0$ such that $r_{n} \leq 2^{-n}$ and

(a) $\rho\left(x, y_{n+1}\right) \leq r_{n+1} \Rightarrow \rho\left(x, y_{n}\right) \leq r_{n}$,

(b) $\rho\left(x_{n+1}, y_{n+1}\right) \geq 2 r_{n+1}$.

(iii) Continuing with (ii), show that the $y_{n}$ form a Cauchy sequence for $\rho$ which does not converge.

(iv) Deduce that $(\mathbb{Q}, d)$ is not homeomorphic to a complete metric space.

Exercise 16.32. We get interesting results when we allow for an interplay between algebra and topology. Consider a topological group, that is to say a group $G$ together with a topology $\tau$ on $G$ such that (if we give $G \times G$ the associated product topology) the multiplication function $m: G \times G \rightarrow G$ (given by $m(x, y)=x y)$ and the inverse function $j: G \rightarrow G\left(\right.$ given by $\left.j^{-1}(x)=x^{-1}\right)$ 
are continuous. Typical examples include the matrix groups such as $S O\left(\mathbb{R}^{3}\right)$ and $U\left(\mathbb{C}^{3}\right.$.

(i) (Homogeneity) Show that that, if $u, v \in G$, there exists a homeomorphism $\phi: G \rightarrow G$ with $\phi(u)=v$.

(ii) Show that $G$ is Hausdorff if and only if $\{e\}$ is closed.

(iii) If $\{e\}$ is closed, show that the diagonal

$$
\Delta G=\{(x, x): x \in G\}
$$

is a closed subgroup of $G \times G$ (i.e both a subgroup and closed in the product topology).

(iv) If $\{e\}$ is closed, show that the centre

$$
Z(G)=\{g: g h=h g \forall h \in G\}
$$

is a closed normal subgroup.

(v) Suppose that $H$ is a subgroup of $G$. Consider the collection $X$ of cosets of $H$. Show that, if we give $X$ the natural quotient topology, the map $\pi: G \rightarrow X$ given by $\pi(g)=g H$ is open (that is to say $\pi$ maps open sets to open sets).

(vi) Show that $X$, as given in (v), is Hausdorff if and only if $H$ is closed in $G$.

\section{Some hints}

Theorem 9.5. [The Heine-Borel Theorem.] Let $\mathbb{R}$ be given its usual (Euclidean) topology. Then the closed bounded interval $[a, b]$ is compact.

Hint. Suppose that $\mathcal{C}$ is an open cover of $[a, b]$. If $\mathcal{C}_{1}$ is a finite subcover of $[a, c]$ and $\mathcal{C}_{2}$ is a finite subcover of $[c, a]$, then $\mathcal{C}_{1} \cup \mathcal{C}_{2}$ is a finite cover of $[a, b]$.

We can use this as a basis for a lion hunting (bisection) argument.

[Return to page 23 or go to a full proof on 74.$]$

Theorem 9.8. If $(X, \tau)$ is Hausdorff, then every compact set is closed.

Hint. Let $K$ be a compact set. If $x \notin K$, then, given any $k \in K$, we know that $k \neq x$ and so, since $X$ is Hausdorff, we can find open sets $U_{k}$ and $V_{k}$ such that

$$
k \in V_{k}, x \in U_{k} \text { and } V_{k} \cap U_{k}=\varnothing .
$$

Now use compactness.

[Return to page 24 or go to a full proof on 76.$]$ 
Theorem 9.18. Let $(X, \tau)$ be a compact and $(Y, \sigma)$ a Hausdorff topological space. If $f: X \rightarrow Y$ is a continuous bijection, then it is a homeomorphism.

Hint. Observe that we need only show that $f(K)$ is closed whenever $K$ is closed.

[Return to page 25 or go to a full proof on 78.$]$

Theorem 10.1. The product of two compact spaces is compact. (More formally, if $(X, \tau)$ and $(Y, \sigma)$ are compact topological spaces and $\lambda$ is the product topology, then $(X \times Y, \lambda)$ is compact.)

Hint. Let $\left\{O_{\alpha}\right\}_{\alpha \in A}$ be an open cover for $X \times Y$. Then given $(x, y) \in X \times Y$ we can find $U_{x, y} \in \tau, V_{x, y} \in \sigma$ and $\alpha(x, y) \in A$ such that

$$
(x, y) \in U_{x, y} \times V_{x, y} \subseteq O_{\alpha(x, y)} .
$$

Now show that, for each $x \in X$, we can find a positive integer $n(x)$ and $y(x, j) \in Y[1 \leq j \leq n(x)]$ such that

$$
\bigcup_{j=1}^{n(x)} V_{x, y(x, j)}=Y .
$$

Now consider the $U_{x}=\bigcap_{j=1}^{n(x)} U_{x, y(x, j)}$.

[Return to page 26 or go to a full proof on 79. .]

Example 11.7. Suppose that $E$ is a connected subset of a topological space $(X, \tau)$. Suppose that $f: E \rightarrow \mathbb{R}$ is 'locally constant' in the sense that, given any $e \in E$, we can find an open neighbourhood $U$ of e such that $f$ is constant on $U \cap E$. Then $f$ is constant.

Hint. There is no loss in generality in taking $E=X$ and $X \neq \varnothing$. Choose an $x_{0} \in X$ and set $c=f\left(x_{0}\right)$. Now consider the sets

$$
U=\{x \in X: f(x)=c\} \text { and } V=\{x \in X: f(x) \neq c\} .
$$

[Return to page 30 or go to a full proof on 83.]

Theorem 11.13. If we give $\mathbb{R}^{n}$ the usual topology, then any open set $\Omega$ which is connected is path-connected.

Hint. Pick $\mathbf{x} \in \Omega$, let $U$ be the set of all points in $\Omega$ which are path-connected to $\mathbf{x}$ and let $V$ be the set of all points in $\Omega$ which are not. We need to prove that $U$ and $V$ are open and to do this we make use of the fact that any point in an open ball is path-connected to the centre of the ball.

[Return to page 31 or go to a full proof on page 86.] 
Theorem 12.4. If the metric space $(X, d)$ is compact, it is sequentially compact.

Hint. Let $x_{n}$ be a sequence in $X$. If it has no convergent subsequence, then, for each $x \in X$, we can find a $\delta(x)>0$ and an $N(x)$ such that $x_{n} \notin B(x, \delta(x))$ for all $n \geq N(x)$.

[Return to page 32 or go to a full proof on page 88.]

Lemma 12.5. Suppose that $(X, d)$ is a sequentially compact metric space and that the collection $U_{\alpha}$ with $\alpha \in A$ is an open cover of $X$. Then there exists a $\delta>0$ such that, given any $x \in X$, there exists an $\alpha(x) \in A$ such that the open ball $B(x, \delta) \subseteq U_{\alpha(x)}$.

Hint. Suppose the first sentence is true and the second sentence false. Then, for each $n \geq 1$, we can find an $x_{n}$ such that $B\left(x_{n}, 1 / n\right) \nsubseteq U_{\alpha}$ for all $\alpha \in A$.

[Return to page 32 or go to a full proof on page 89.]

Theorem 12.6. If the metric space $(X, d)$ is sequentially compact, it is compact.

Hint. Let $\left(U_{\alpha}\right)_{\alpha \in A}$ be an open cover and let $\delta$ be defined as in Lemma 12.5. The $B(x, \delta)$ form a cover of $X$. If they have no finite subcover then, given $x_{1}, x_{2}, \ldots x_{n}$, we can find an $x_{n+1} \notin \bigcup_{j=1}^{n} B\left(x_{j}, \delta\right)$.

[Return to page 32 or go to a full proof on page 89.]

\section{Some proofs}

Exercise 2.2. If $d: X^{2} \rightarrow \mathbb{R}$ is a function with the following properties:-

(ii) $d(x, y)=0$ if and only if $x=y$,

(iii) $d(x, y)=d(y, x)$ for all $x, y \in X$,

(iv) $d(x, y)+d(y, z) \geq d(x, z)$ for all $x, y, z \in X$,

show that $d$ is a metric on $X$.

Solution. Setting $z=x$ in condition (iv) and using (iii) and (ii), we have

$$
2 d(x, y)=d(x, y)+d(y, x) \geq d(x, x)=0
$$

so $d(x, y) \geq 0$.

[Return to page 3.] 
Exercise 2.4. Let $X=\{a, b, c\}$ with $a, b$ and $c$ distinct. Write down functions $d_{j}: X^{2} \rightarrow \mathbb{R}$ satisfying condition (i) of Definition 2.1 such that

(1) $d_{1}$ satisfies conditions (ii) and (iii) but not (iv).

(2) $d_{2}$ satisfies conditions (iii) and (iv) but it is not true that $x=y$ implies $d(x, y)=0$.

(3) $d_{3}$ satisfies conditions (iii) and (iv) and $x=y$ implies $d_{3}(x, y)=0$. but it is not true that $d_{3}(x, y)=0$ implies $x=y$.

(4) $d_{4}$ satisfies conditions (ii) and (iv) but not (iii).

You should verify your statements.

Solution. Here are some possible choices.

(1) Take $d_{1}(x, x)=0$ for all $x \in X, d_{1}(a, b)=d_{1}(b, a)=d_{1}(a, c)=$ $d_{1}(c, a)=1$ and $d_{1}(b, c)=d_{1}(c, b)=3$. Conditions (ii) and (iii) hold by inspection, but

$$
d_{1}(b, a)+d_{1}(a, c)=2<3=d_{1}(b, c) .
$$

(2) Take $d_{2}(x, x)=1$ and $d_{2}(x, y)=2$ if $x \neq y$. Condition (ii) fails and condition (iii) holds by inspection. We observe that

$$
d_{2}(x, y)+d_{2}(y, z) \geq 1+1=2 \geq d_{2}(x, z)
$$

so the triangle law holds.

(3) Take $d_{2}(x, y)=0$ for all $x, y \in X$.

(4) Take $d_{4}(x, x)=0$ for all $x \in X, d_{4}(a, b)=d_{4}(b, a)=d_{4}(a, c)=$ $d_{4}(c, a)=1$ and $d_{1}(b, c)=d_{1}(c, b)=\frac{5}{4}$. Conditions (ii) holds, and condition (iii) fails by inspection and

$$
\begin{array}{ll}
d(x, y)+d(y, z)=d(x, y)=d(x, z) \geq d(x, z) & \text { if } y=z, \\
d(x, y)+d(y, z)=d(y, z)=d(x, z) \geq d(x, z) & \text { if } x=y, \\
d(x, y)+d(y, z) \geq 1+1=2 \geq \frac{5}{4} \geq d(x, z) & \text { otherwise, }
\end{array}
$$

so the triangle law holds.

[Return to page 4.]

Exercise 2.8. If $(V,\|\|)$ is a normed vector space, then the condition

$$
d(\mathbf{u}, \mathbf{v})=\|\mathbf{u}-\mathbf{v}\|
$$

defines a metric d on $V$.

Solution. We observe that

$$
d(\mathbf{u}, \mathbf{v})=\|\mathbf{u}-\mathbf{v}\| \geq 0
$$


and

$$
d(\mathbf{u}, \mathbf{u})=\|\mathbf{0}\|=\|0 \mathbf{0}\|=|0|\|\mathbf{0}\|=0\|\mathbf{0}\|=0 .
$$

Further, if $d(\mathbf{u}, \mathbf{v})=0$, then $\|\mathbf{u}-\mathbf{v}\|=0$ so $\mathbf{u}-\mathbf{v}=\mathbf{0}$ and $\mathbf{u}=\mathbf{v}$. We also observe that

$$
d(\mathbf{u}, \mathbf{v})=\|\mathbf{u}-\mathbf{v}\|=\|(-1)(\mathbf{v}-\mathbf{u})\|=|-1|\|\mathbf{v}-\mathbf{u}\|=d(\mathbf{v}, \mathbf{u})
$$

and

$$
\begin{aligned}
d(\mathbf{u}, \mathbf{v})+d(\mathbf{v}, \mathbf{w}) & =\|\mathbf{u}-\mathbf{v}\|+\|\mathbf{v}-\mathbf{w}\| \\
& \geq\|(\mathbf{u}-\mathbf{v})+(\mathbf{v}-\mathbf{w})\| \\
& =\|\mathbf{u}-\mathbf{w}\|=d(\mathbf{u}, \mathbf{w}) .
\end{aligned}
$$

[Return to page 5.]

Lemma 2.10. The discrete metric on $X$ is indeed a metric.

Proof. The only non-evident condition is the triangle law. But

$$
\begin{array}{ll}
d(x, y)+d(y, z)=d(x, y)=d(x, z) \geq d(x, z) & \text { if } y=z \\
d(x, y)+d(y, z)=d(y, z)=d(x, z) \geq d(x, z) & \text { if } x=y, \\
d(x, y)+d(y, z) \geq 1+1=2 \geq 1 \geq d(x, z) & \text { otherwise, }
\end{array}
$$

so we are done.

[Return to page 5.]

Lemma 2.16. Show that the British Rail express metric and the British Rail stopping metric are indeed metrics.

Solution. We show that the British Rail stopping metric is indeed a metric. The case of the British Rail express metric is left to the reader.

Let $d$ be the British rail stopping metric on $\mathbb{R}^{2}$. It is easy to see that $d(\mathbf{u}, \mathbf{v}) \geq 0$ and that $d(\mathbf{u}, \mathbf{v})=d(\mathbf{v}, \mathbf{u})$. Since $\mathbf{u}$ and $\mathbf{u}$ are linearly dependent,

$$
d(\mathbf{u}, \mathbf{u})=\|\mathbf{u}-\mathbf{u}\|_{2}=\|\mathbf{0}\|_{2}=0 .
$$

If $d(\mathbf{u}, \mathbf{v})=0$, then we know that at least one of the following statements is true

(1) $\|\mathbf{u}-\mathbf{v}\|_{2}=0$ and so $\mathbf{u}-\mathbf{v}=\mathbf{0}$,

(2) $\|\mathbf{u}\|_{2}+\|\mathbf{v}\|_{2}=0$ and so $\|\mathbf{u}\|_{2}=\|\mathbf{v}\|_{2}=0$, whence $\mathbf{u}=\mathbf{v}=\mathbf{0}$.

In either case $\mathbf{u}=\mathbf{v}$ as required. 
It only remains to prove the triangle inequality. Observe that, if $\mathbf{v}$ and $\mathbf{w}$ are not linearly dependent,

$$
d(\mathbf{u}, \mathbf{v})+d(\mathbf{v}, \mathbf{w}) \geq\|\mathbf{u}-\mathbf{v}\|_{2}+\|\mathbf{v}\|_{2}+\|\mathbf{w}\|_{2} \geq\|\mathbf{u}\|_{2}+\|\mathbf{w}\|_{2} \geq d(\mathbf{u}, \mathbf{w}) .
$$

By similar reasoning

$$
d(\mathbf{u}, \mathbf{v})+d(\mathbf{v}, \mathbf{w}) \geq d(\mathbf{u}, \mathbf{w})
$$

if $\mathbf{u}$ and $\mathbf{v}$ are not linearly dependent. Finally, if $\mathbf{u}$ and $\mathbf{v}$ are linearly dependent and $\mathbf{v}$ and $\mathbf{w}$ are linearly dependent, then $\mathbf{u}$ and $\mathbf{w}$ are linearly dependent so

$$
d(\mathbf{u}, \mathbf{v})+d(\mathbf{v}, \mathbf{w})=\|\mathbf{u}-\mathbf{v}\|_{2}+\|\mathbf{v}-\mathbf{w}\|_{2} \geq\|\mathbf{u}-\mathbf{w}\|_{2}=d(\mathbf{u}, \mathbf{w}) .
$$

Thus the triangle law holds.

[Return to page 7.]

Lemma 3.3. [The composition law.] If $(X, d)$ and $(Y, \rho)$ and $(Z, \sigma)$ are metric spaces and $g: X \rightarrow Y, f: Y \rightarrow Z$ are continuous, then so is the composition $f g$.

Proof. Let $\epsilon>0$ be given and let $x \in X$. Since $f$ is continuous, we can find a $\delta_{1}>0$ (depending on $\epsilon$ and $f g(x)=f(g(x))$ with

$$
\sigma(f(g(x)), f(y))<\epsilon \text { whenever } \rho(g(x), y)<\delta_{1} .
$$

Since $g$ is continuous, we can find a $\delta_{2}>0$ such that

$$
\rho(g(x), g(t))<\delta_{1} \text { whenever } d(x, t)<\delta_{2} .
$$

We now have

$$
\sigma(f(g(x)), f(g(t)))<\epsilon \text { whenever } d(x, t)<\delta_{2}
$$

as required.

[Return to page 7.]

Exercise 3.4. Let $\mathbb{R}$ and $\mathbb{R}^{2}$ have their usual (Euclidean) metric.

(i) Suppose that $f: \mathbb{R} \rightarrow \mathbb{R}$ and $g: \mathbb{R} \rightarrow \mathbb{R}$ are continuous. Show that the map $(f, g): \mathbb{R}^{2} \rightarrow \mathbb{R}^{2}$ is continuous.

(ii) Show that the map $M: \mathbb{R}^{2} \rightarrow \mathbb{R}$ given by $M(x, y)=x y$ is continuous.

(iii) Use the composition law to show that the map $m: \mathbb{R}^{2} \rightarrow \mathbb{R}$ given by $m(x, y)=f(x) g(y)$ is continuous. 
Solution. (i) Let $(x, y) \in \mathbb{R}^{2}$. Given $\epsilon>0$, we can find $\delta_{1}>0$ such that

$$
|f(x)-f(s)|<\epsilon / 2 \text { whenever }|x-s|<\delta_{1}
$$

and $\delta_{2}>0$ such that

$$
|g(y)-g(t)|<\epsilon / 2 \text { whenever }|y-t|<\delta_{2} .
$$

If we set $\delta=\min \left(\delta_{1}, \delta_{2}\right)$, then $\|(x, y)-(s, t)\|_{2}<\delta$ implies

$$
|x-s|<\delta \leq \delta_{1} \text { and }|y-t|<\delta \leq \delta_{2}
$$

so that

$$
|f(x)-f(s)|<\epsilon / 2 \text { and }|g(y)-g(t)|<\epsilon / 2
$$

whence

$$
\begin{aligned}
\|(f(x), g(y))-(f(s), g(t))\|_{2} & \leq\|(f(x), 0)-(f(s), 0)\|_{2}+\|(0, g(y))-(0, g(t))\|_{2} \\
& =|f(x)-f(s)|+|g(y)-g(t)|<\epsilon
\end{aligned}
$$

as required.

[Return to page 7.]

Example 3.6. (i) Let $(X, d)$ be a metric space. If $r>0$, then

$$
B(x, r)=\{y: d(x, y)<r\}
$$

is open.

(ii) If we work in $\mathbb{R}^{n}$ with the Euclidean metric, then the one point set $\{\mathbf{x}\}$ is not open.

(iii) If $(X, d)$ is a discrete metric space, then

$$
\{x\}=B(x, 1 / 2)
$$

and all subsets of $X$ are open.

Proof. (i) If $y \in B(x, r)$, then $\delta=r-d(x, y)>0$ and, whenever $d(z, y)<\delta$, the triangle inequality gives us

$$
d(x, z) \leq d(x, y)+d(y, z)<r
$$

so $z \in B(x, r)$. Thus $B(x, r)$ is open.

(ii) Choose $\mathbf{e} \in \mathbb{R}^{n}$ with $\|\mathbf{e}\|_{2}=1$. (We could take $\mathbf{e}=(1,0,0, \ldots, 0)$.) If $\delta>0$, then, setting $\mathbf{y}=\mathbf{x}+(\delta / 2) \mathbf{e}$, we have $\|\mathbf{x}-\mathbf{y}\|_{2}<\delta$, yet $\mathbf{y} \notin\{\mathbf{x}\}$. Thus $\{\mathbf{x}\}$ is not closed.

(iii) Observe that $d(x, x)=0<1 / 2$ and $d(x, y)=1>1 / 2$ for $x \neq y$. If $x \in E$ then $d(x, y)<1 / 2$ implies $y=x \in E$, so $E$ is open.

[Return to page 9.] 
Theorem 3.7. If $(X, d)$ is a metric space, then the following statements are true.

(i) The empty set $\varnothing$ and the space $X$ are open.

(ii) If $U_{\alpha}$ is open for all $\alpha \in A$, then $\bigcup_{\alpha \in A} U_{\alpha}$ is open. (In other words, the union of open sets is open.)

(iii) If $U_{j}$ is open for all $1 \leq j \leq n$, then $\bigcap_{j=1}^{n} U_{j}$ is open.

Proof. (i) Since there are no points $e$ in $\varnothing$, the statement

$$
x \in \varnothing \text { whenever } d(x, e)<1
$$

holds for all $e \in \varnothing$. Since every point $x$ belongs to $X$, the statement

$$
x \in X \text { whenever } d(x, e)<1
$$

holds for all $e \in X$.

(ii) If $e \in \bigcup_{\alpha \in A} U_{\alpha}$, then we can find a particular $\alpha_{1} \in A$ with $e \in U_{\alpha_{1}}$. Since $U_{\alpha_{1}}$ is open, we can find a $\delta>0$ such that

$$
x \in U_{\alpha_{1}} \text { whenever } d(x, e)<\delta .
$$

Since $U_{\alpha_{1}} \subseteq \bigcup_{\alpha \in A} U_{\alpha}$

$$
x \in \bigcup_{\alpha \in A} U_{\alpha} \text { whenever } d(x, e)<\delta .
$$

Thus $\bigcup_{\alpha \in A} U_{\alpha}$ is open.

(iii) If $e \in \bigcap_{j=1}^{n} U_{j}$, then $e \in U_{j}$ for each $1 \leq j \leq n$. Since $U_{j}$ is open, we can find a $\delta_{j}>0$ such that

$$
x \in U_{j} \text { whenever } d(x, e)<\delta_{j} .
$$

Setting $\delta=\min _{1 \leq j \leq n} \delta_{j}$, we have $\delta>0$ and

$$
x \in U_{j} \text { whenever } d(x, e)<\delta
$$

for all $1 \leq j \leq n$. Thus

$$
x \in \bigcap_{j=1}^{n} U_{j} \text { whenever } d(x, e)<\delta
$$

and we have shown that $\bigcap_{j=1}^{n} U_{j}$ is open.

[Return to page 9.] 
Theorem 3.9. Let $(X, d)$ and $(Y, \rho)$ be metric spaces. A function $f: X \rightarrow Y$ is continuous if and only if $f^{-1}(U)$ is open in $X$ whenever $U$ is open in $Y$.

Proof. Suppose first that $f$ is continuous and that $U$ is open in $Y$. If $x \in$ $f^{-1}(U)$, then we can find a $y \in U$ with $f(x)=y$. Since $U$ is open in $Y$, we can find an $\epsilon>0$ such that

$$
z \in U \text { whenever } \rho(y, z)<\epsilon \text {. }
$$

Since $f$ is continuous, we can find a $\delta>0$ such that

$$
\rho(y, f(w))=\rho(f(x), f(w))<\epsilon \text { whenever } d(x, w)<\delta .
$$

Thus

$$
f(w) \in U \text { whenever } d(x, w)<\delta .
$$

In other words,

$$
w \in f^{-1}(U) \text { whenever } d(x, w)<\delta .
$$

We have shown that $f^{-1}(U)$ is open.

We now seek the converse result. Suppose that $f^{-1}(U)$ is open in $X$ whenever $U$ is open in $Y$. Suppose $x \in X$ and $\epsilon>0$. We know that the open ball

$$
B(f(x), \epsilon)=\{y \in Y: \rho(f(x), y)<\epsilon\}
$$

is open. Thus $x \in f^{-1} B(f(x), \epsilon)$ and $f^{-1} B(f(x), \epsilon)$ is open. It follows that there is a $\delta>0$ such that

$$
w \in f^{-1} B(f(x), \epsilon) \text { whenever } d(x, w)<\delta,
$$

so, in other words,

$$
\rho(f(x), f(w))<\epsilon \text { whenever } d(x, w)<\delta .
$$

Thus $f$ is continuous.

[Return to page 9.]

Example 3.10. Let $X=\mathbb{R}$ and $d$ be the discrete metric. Let $Y=\mathbb{R}$ and $\rho$ be the usual (Euclidean) metric.

(i) If we define $f: X \rightarrow Y$ by $f(x)=x$, then $f$ is continuous but there exist open sets $U$ in $X$ such that $f(U)$ is not open.

(ii) If we define $g: Y \rightarrow X$ by $g(y)=y$, then $g$ is not continuous but $g(V)$ is open in $X$ whenever $V$ is open in $Y$. 
Proof. Since every set is open in $X$, we have $f^{-1}(V)=g(V)$ open for every $V$ in $Y$ and so, in particular, for every open set. Thus $f$ is continuous.

We observe that $U=\{0\}$ is open in $X$ and $g^{-1}(U)=f(U)=U=\{0\}$ is not open in $Y$. Thus $g$ is not continuous.

[Return to page 10.]

Exercise 3.11. Consider $\mathbb{R}^{2}$. For each of the British rail express and British rail stopping metrics:-

(i) Describe the open balls. (Consider both large and small radii.)

(ii) Describe the open sets as well as you can. (There is a nice description for the British rail express metric.) Give reasons for your answers.

Solution. We start with the British rail express metric. Write

$$
B_{E}(\delta)=\left\{\mathbf{x}:\|\mathbf{x}\|_{2}<\delta\right\}
$$

for the Euclidean ball centre $\mathbf{0}[\delta>0]$. If $0<r<\|\mathbf{x}\|_{2}$, then

$$
B(\mathbf{x}, r)=\{\mathbf{x}\}
$$

If $\|\mathbf{x}\|_{2}>r>0$, then

$$
B(\mathbf{x}, r)=\{\mathbf{x}\} \cup B_{E}(r-\|\mathbf{x}\|) .
$$

Since open balls are open and the union of open sets is open, we deduce that every set not containing $\mathbf{0}$ and every set containing $B_{E}(\delta)$ for some $\delta>0$ is open.

On the other hand, if $U$ is open and $\mathbf{0} \in U$ then $U$ must contain $B_{E}(\delta)$ for some $\delta>0$. It follows that the collection of sets described in the last sentence of the previous paragraph constitute the open sets for the British rail express metric.

We turn now to the stopping metric. We observe that

$$
B(\mathbf{0}, r)=B_{E}(r)
$$

for $r>0$. If $\mathbf{x} \neq \mathbf{0}$ and $0<r<\|\mathbf{x}\|_{2}$, then

$$
B(\mathbf{x}, r)=\left\{\lambda \frac{\mathbf{x}}{\|\mathbf{x}\|_{2}}: \lambda \in\left(\|\mathbf{x}\|_{2}-r,\|\mathbf{x}\|_{2}+r\right)\right\} .
$$

If $\mathbf{x} \neq \mathbf{0}$ and $\|\mathbf{x}\|_{2}>r>0$, then

$$
B(\mathbf{x}, r)=\left\{\lambda \frac{\mathbf{x}}{\|\mathbf{x}\|_{2}}: \lambda \in\left(0,\|\mathbf{x}\|_{2}+r\right)\right\} \cup B_{E}(r-\|\mathbf{x}\|) .
$$


A similar argument to the previous paragraph shows that the open sets are precisely the unions of sets of the form

$$
l(\mathbf{e},(a, b))=\{\lambda \mathbf{e}: \lambda \in(a, b)\}
$$

where $\mathbf{e}$ is a unit vector and $0 \leq a<b$ together with some $B_{E}(\delta)$ with $\delta>0$.

[Return to page 10.]

Lemma 4.2. Consider a metric space $(X, d)$. If a sequence $x_{n}$ has a limit, then that limit is unique.

Proof. Suppose $x_{n} \rightarrow x$ and $x_{n} \rightarrow y$. Then, given any $\epsilon>0$, we can find $N_{1}$ and $N_{2}$ such that

$$
d\left(x_{n}, x\right)<\epsilon / 2 \text { for all } n \geq N_{1} \text { and } d\left(x_{n}, y\right)<\epsilon / 2 \text { for all } n \geq N_{2} .
$$

Taking $N=\max \left(N_{1}, N_{2}\right)$, we obtain

$$
d(x, y) \leq d\left(x_{N}, x\right)+d\left(x_{N}, y\right)<\epsilon / 2+\epsilon / 2=\epsilon .
$$

Since $\epsilon$ was arbitrary, $d(x, y)=0$ and $x=y$.

[Return to page 11.]

Exercise 4.3. Consider two metric spaces $(X, d)$ and $(Y, \rho)$. Show that a function $f: X \rightarrow Y$ is continuous if and only if, whenever $x_{n} \in X$ and $x_{n} \rightarrow x$ as $n \rightarrow \infty$, we have $f\left(x_{n}\right) \rightarrow f(x)$

Solution. Suppose that $f$ is continuous and $x_{n} \rightarrow x$. Then, given $\epsilon>0$, we can find a $\delta>0$ such that

$$
d(z, x)<\epsilon \Rightarrow \rho(f(z), f(x))<\delta
$$

and then find an $N$ such that

$$
n \geq N \Rightarrow d\left(x_{n}, x\right)<\epsilon
$$

and so

$$
n \geq N \Rightarrow \rho(f(z), f(x))<\delta .
$$

Thus $f\left(x_{n}\right) \rightarrow f(x)$.

If $f$ is not continuous, we can find a $\delta>0$ such that, given any $\epsilon>0$, there exists an $z \in X$ with $d(z, x)<\epsilon$ and $\rho(f(z), f(x))>\delta$. In particular, we can find $x_{n} \in X$ such that $d\left(x_{n}, x\right)<1 / n$ but $\rho\left(f\left(x_{n}\right), f(x)\right)>\delta$. Thus $x_{n} \rightarrow x$, but $f\left(x_{n}\right) \not \rightarrow f(x)$.

[Return to page 11] 
Theorem 4.7. Let $(X, d)$ be a metric space. A set $F$ in $X$ is closed if and only if its complement is open.

Proof. Only if Suppose that $F$ is closed and $E=X \backslash F$. If $E$ is not open, we can find an $e \in E$ such that $B(e, \delta) \cap F \neq \varnothing$ for all $\delta>0$. In particular, we can find $x_{n} \in F$ such that $d\left(x_{n}, e\right)<1 / n$ for each $n \geq 1$. Since $x_{n} \rightarrow e$ and $F$ is closed, we have $e \in F$ contradicting our initial assumption that $e \in E$. Thus $E$ is open.

If We now establish the converse. Suppose $E$ is open and $F=X \backslash E$. Suppose $x_{n} \in F$ and $x_{n} \rightarrow x$. If $x \in E$, then, since $E$ is open we can find a $\delta>0$ such that $B(x, \delta) \subseteq E$. Thus $d\left(x_{n}, x\right) \geq \delta$ for all $n$ which is absurd. Thus $x \in F$ and $F$ is closed.

[Return to page 11.]

Theorem 4.8. If $(X, d)$ is a metric space, then the following statements are true.

(i) The empty set $\varnothing$ and the space $X$ are closed.

(ii) If $F_{\alpha}$ is closed for all $\alpha \in A$, then $\bigcap_{\alpha \in A} F_{\alpha}$ is closed. (In other words the intersection of closed sets is closed.)

(iii) If $F_{j}$ is closed for all $1 \leq j \leq n$, then $\bigcup_{j=1}^{n} F_{j}$ is open.

Proof. (i) Observe that $\varnothing=X \backslash X$ and $X=X \backslash \varnothing$.

(ii) Since $F_{\alpha}$ is closed, $X \backslash F_{\alpha}$ is open for all $\alpha \in A$. It follows that

$$
X \backslash \bigcap_{\alpha \in A} F_{\alpha}=\bigcup_{\alpha \in A}\left(X \backslash F_{\alpha}\right)
$$

is open and so $\bigcap_{\alpha \in A} F_{\alpha}$ is closed.

(iii) Since $F_{j}$ is closed, $X \backslash F_{j}$ is open for all $1 \leq j \leq n$. It follows that

$$
X \backslash \bigcup_{j=1}^{n} F_{j}=\bigcap_{j=1}^{n}\left(X \backslash F_{j}\right)
$$

is open and so $\bigcup_{j=1}^{n} F_{j}$ is closed.

[Return to page 12.]

Theorem 4.9. Let $(X, d)$ and $(Y, \rho)$ be metric spaces. A function $f: X \rightarrow Y$ is continuous if and only if $f^{-1}(F)$ is closed in $X$ whenever $F$ is closed in $Y$.

Proof. If Suppose that $f$ is continuous. If $F$ is closed in $Y$, then $Y \backslash F$ is open, so

$$
X \backslash f^{-1}(F)=f^{-1}(Y \backslash F)
$$


is open. Thus $f^{-1}(F)$ is closed.

Only if Suppose $f^{-1}(F)$ is closed whenever $F$ is. If $U$ is open in $Y$, then $Y \backslash U$ is closed, so

$$
X \backslash f^{-1}(U)=f^{-1}(Y \backslash U)
$$

is closed. Thus $f^{-1}(U)$ is open. We have shown that $f$ is continuous.

[Return to page 12.]

Exercise 5.6. Write $\mathcal{P}(Y)$ for the collection of subsets of $Y$. If $X$ has three elements, how many elements does $\mathcal{P}(\mathcal{P}(X))$ have?

How many topologies are there on $X$ ?

Solution. If $Y$ has $n$ elements $\mathcal{P}(Y)$ has $2^{n}$ elements so $\mathcal{P}(\mathcal{P}(X))$ has $2^{2^{3}}=$ $2^{8}=256$ elements.

Let $X=\{x, y, z\}$. We set out the types of possible topologies below.

$$
\begin{array}{c|c}
\text { type } & \text { number of this type } \\
\{\varnothing, X\} & 1 \\
\{\varnothing,\{x\}, X\} & 3 \\
\{\varnothing,\{x\},\{y\},\{x, y\}, X\} & 3 \\
\mathcal{P}(X) & 1 \\
\{\varnothing,\{x, y\}, X\} & 3 \\
\{\varnothing,\{x\},\{x, y\}, X\} & 6 \\
\{\varnothing,\{z\},\{x, y\}, X\} & 3 \\
\{\varnothing,\{x\},\{z\},\{x, y\},\{x, z\}, X\} & 6 \\
\{\varnothing,\{x\},\{x, y\},\{x, z\}, X\} & 3
\end{array}
$$

There are that 29 distinct topologies on $X$.

The moral of this question is that although there are far fewer topologies than simple collections of subsets and even fewer different types (nonhomeomorphic topologies in later terminology) there are still quite a lot even for spaces of three points.

[Return to page 13.]

Lemma 6.3. Let $(X, \tau)$ be a topological space and $A$ a subset of $X$.

(i) Int $A=\{x \in A: \exists U \in \tau$ with $x \in U \subseteq A\}$.

(ii) Int $A$ is the unique $V \in \tau$ such that $V \subseteq A$ and, if $W \in \tau$ and $V \subseteq W \subseteq A$, then $V=W$. (Informally, Int $A$ is the largest open set contained in $A$.)

Proof. (i) This is just the observation that

$$
\begin{aligned}
\operatorname{Int} A & =\bigcup\{U \in \tau: U \subseteq A\} \\
& =\{x \in A: \exists U \in \tau \text { with } x \in U \subseteq A\}
\end{aligned}
$$


(ii) Since

$$
\text { Int } A=\bigcup\{U \in \tau: U \subseteq A\}
$$

we know that Int $A \subseteq A$. Since the union of open sets is open, Int $A \in \tau$. If $W \in \tau$ and $W \subseteq A$ then

$$
\operatorname{Int} A=\bigcup\{U \in \tau: U \subseteq A\} \supseteq W
$$

so, if $W \supseteq \operatorname{Int} A, W=\int A$.

To prove uniqueness, suppose that $V^{\prime}$ is an open subset of $A$ and has the property that, if $U$ in $\tau$ and $V^{\prime} \subseteq U \subseteq A$. then $V^{\prime}=U$. Since $V^{\prime}$ is an open subset of $A$, we have $V^{\prime} \subseteq \operatorname{Int} A \subseteq A$ so $V^{\prime}=\operatorname{Int} A$.

[Return to page 15.]

Lemma 6.6. Let $(X, d)$ be a metric space and $A$ a subset of $X$. Then $\mathrm{Cl} A$ consists of all those $x$ such that we can find $x_{n} \in A$ with $d\left(x, x_{n}\right) \rightarrow 0$. (In old fashioned terminology, the closure of $A$ is its set of limit points.)

Proof. Suppose that $x_{n} \in A$ with $d\left(x, x_{n}\right) \rightarrow 0$. Then, since $A \subseteq \mathrm{Cl} A$, $x_{n} \in \mathrm{Cl} A$ and so, since $\mathrm{Cl} A$ is closed, $x \in \mathrm{Cl} A$.

Suppose conversely that $x \in \mathrm{Cl} A$. Since $\mathrm{Cl} A=X \backslash \operatorname{Int} A^{c}$, we know that the open ball $B(x, 1 / n)$ of radius $1 / n$ and centre $x$ cannot lie entirely within $A^{c}$, so there exists an $x_{n} \in B(x, 1 / n) \cap A$. We have $d\left(x, x_{n}\right) \rightarrow 0$, so we are done.

[Return to page 15.]

Exercise 6.8. (i) Let $(X, \tau)$ be a topological space and $(Y, d)$ a metric space. If $f, g: X \rightarrow Y$ are continuous and $f(x)=g(x)$ for all $x \in A$, where $A$ is dense in $X$, show that $f(x)=g(x)$ for all $x \in X$.

(ii) Consider the unit interval $[0,1]$ with the Euclidean metric and $A=$ $[0,1] \cap \mathbb{Q}$ with the inherited metric. Exhibit, with proof, a continuous map $f: A \rightarrow \mathbb{R}$ (where $\mathbb{R}$ has the standard metric) such that there does not exist a continuous map $\tilde{f}:[0,1] \rightarrow \mathbb{R}$ with $\tilde{f}(x)=f(x)$ for all $x \in[0,1]$.

Solution. (i) Suppose $f(b) \neq g(b)$ for some $b \in X$. We can find open sets $U$ and $V$ such that $f(b) \in U, g(b) \in V$ and $U \cap V=\varnothing$. Now $f^{-1}(U)$ is open as is $g^{-1}(U)$ so $b \in f^{-1}(U) \cap g^{-1}(V) \in \tau$. Since $A$ is dense we can find an $a \in A$ with $a \in f^{-1}(U) \cap g^{-1}(V)$. Now $f(a) \in U$ and $g(a) \in V$, but $f(a)=g(a)$, so $f(a) \in U \cap V$, contradicting our earlier assertion that $U \cap V=\varnothing$. The result follows by contradiction.

(ii) We observe that $x \in A \Rightarrow x^{2} \neq \frac{1}{2}$. If $x \in A$, set

$$
f(x)= \begin{cases}0 & \text { if } x^{2}<\frac{1}{2} \\ 1 & \text { otherwise }\end{cases}
$$


Observe that, if $y \in A$ and $y^{2}<\frac{1}{2}$, we can find a $\delta>0$ such that

$$
|y-x|<\delta \Rightarrow x^{2}<\frac{1}{2} \Rightarrow f(x)=f(y) .
$$

Similarly if $y \in A$ and $y^{2}>\frac{1}{2}$ we can find a $\delta>0$ such that

$$
|y-x|<\delta \Rightarrow x^{2}>\frac{1}{2} \Rightarrow f(x)=f(y)
$$

Thus $f$ is continuous.

Suppose that $\tilde{f}:[0,1] \rightarrow \mathbb{R}$. is such that $\tilde{f}(x)=f(x)$ for all $x \in A$. Choose $p_{n}, q_{n} \in A$ such that $p_{n}^{2}>\frac{1}{2}>q_{n}^{2}$ and $\left|p_{n}-2^{-1 / 2}\right|,\left|q_{n}-2^{-1 / 2}\right| \rightarrow 0$. Then

$$
\left|f\left(p_{n}\right)-f\left(2^{-1 / 2}\right)\right|+\left|f\left(q_{n}\right)-f\left(2^{-1 / 2}\right)\right| \geq\left|f\left(p_{n}\right)-f\left(q_{n}\right)\right|=1,
$$

so $f$ cannot be continuous.

[Return to page 16.]

Example 7.4. Let $X=\mathbb{R}$ and let d be the usual metric on $\mathbb{R}$. Let $Y=(0,1)$ (the open interval with end points 0 and 1 ) and let $\rho$ be the usual metric on $(0,1)$. Then $(X, d)$ and $(Y, \rho)$ are homeomorphic as topological spaces, but $(X, d)$ is complete and $(Y, \rho)$ is not.

Proof. We know from first year analysis that $f(x)=\tan (\pi(y-1 / 2))$ is a bijective function $f: Y \rightarrow X$ which is continuous with continuous inverse. (Recall that a strictly increasing continuous function has continuous inverse.) Thus $(X, d)$ and $(Y, \rho)$ are homeomorphic. We know that $(X, d)$ is complete by the general principle of convergence.

However, $1 / n$ is a Cauchy sequence in $Y$ with no limit in $Y$. (If $y \in(0,1)$, then there exists an $N$ with $y>N^{-1}$. If $m \geq 2 N$, then $|1 / m-y| \geq 1 /(2 N)$ so $1 / n \nrightarrow y$.)

[Return to page 17.]

Lemma 7.5. Let $X$ be a space and let $\mathcal{H}$ be a collection of subsets of $X$. Then there exists a unique topology $\tau_{\mathcal{H}}$ such that

(i) $\tau_{\mathcal{H}} \supseteq \mathcal{H}$, and

(ii) if $\tau$ is a topology with $\tau \supseteq \mathcal{H}$, then $\tau \supseteq \tau_{\mathcal{H}}$.

Proof. The proof follows a standard pattern, which is worth learning.

Uniqueness Suppose that $\sigma$ and $\sigma^{\prime}$ are topologies such that

(i) $\sigma \supseteq \mathcal{H}$

(ii) if $\tau$ is a topology with $\tau \supseteq \mathcal{H}$, then $\tau \supseteq \sigma$,

(i) $\sigma^{\prime} \supseteq \mathcal{H}$,

(ii) ${ }^{\prime}$ if $\tau$ is a topology with $\tau \supseteq \mathcal{H}$, then $\tau \supseteq \sigma^{\prime}$. 
By (i) and (ii)', we have $\sigma \supseteq \sigma^{\prime}$ and by (i)' and (ii), we have $\sigma^{\prime} \supseteq \sigma$. Thus $\sigma=\sigma^{\prime}$.

Existence Let $T$ be the set of topologies $\tau$ with $\tau \supseteq \mathcal{H}$. Since the discrete topology contains $\mathcal{H}, T$ is non-empty. Set

$$
\tau_{\mathcal{H}}=\bigcap_{\tau \in T} \tau
$$

By construction, $\tau_{\mathcal{H}} \supseteq \mathcal{H}$ and $\tau \supseteq \tau_{\mathcal{H}}$ whenever $\tau \in T$. Thus we need only show that $\tau_{\mathcal{H}}$ is a topology and this we now do.

(a) $\varnothing, X \in \tau$ for all $\tau \in T$, so $\varnothing, X \in \tau_{\mathcal{H}}$.

(b) If $U_{\alpha} \in \tau_{\mathcal{H}}$, then $U_{\alpha} \in \tau$ for all $\alpha \in A$ and so $\bigcup_{\alpha \in A} U_{\alpha} \in \tau$ for all $\tau \in T$, whence $\bigcup_{\alpha \in A} U_{\alpha} \in \tau_{\mathcal{H}}$.

(c) If $U_{j} \in \tau_{\mathcal{H}}$, then $U_{j} \in \tau$ for all $1 \leq j \leq n$ and so $\bigcap_{j=1}^{n} U_{j} \in \tau$ for all $\tau \in T$, whence $\bigcap_{j=1}^{n} U_{j} \in \tau_{\mathcal{H}}$.

Thus $\tau_{\mathcal{H}}$ is a topology, as required.

[Return to page 17.]

Lemma 7.8. If $(X, \tau)$ is a topological space and $Y \subseteq X$, then the subspace topology $\tau_{Y}$ on $Y$ is the collection of sets $Y \cap U$ with $U \in \tau$.

Proof. Let $j: Y \rightarrow X$ be the inclusion map given by $j(y)=y$ for all $y \in Y$. Write

$$
\sigma=\{Y \cap U: U \in \tau\} .
$$

Since $Y \cap U=j^{-1}(U)$, we know that $\tau_{Y}$ is the smallest topology containing $\sigma$ and that the result will follow if we show that $\sigma$ is a topology on $Y$. The following observations show this and complete the proof.

(a) $\varnothing=Y \cap \varnothing$ and $Y=Y \cap X$.

(b) $\bigcup_{\alpha \in A}\left(Y \cap U_{\alpha}\right)=Y \cap \bigcup_{\alpha \in A} U_{\alpha}$.

(c) $\bigcap_{j=1}^{n}\left(Y \cap U_{j}\right)=Y \cap \bigcap_{j=1}^{n} U_{j}$.

[Return to page 18.]

Lemma 7.12. Let $(X, \tau)$ and $(Y, \sigma)$ be topological spaces and $\lambda$ the product topology on $X \times Y$. Then $O \in \lambda$ if and only if, given $(x, y) \in O$, we can find $U \in \tau$ and $V \in \sigma$ such that

$$
(x, y) \in U \times V \subseteq O
$$

Proof. Let $\mu$ be the collection of subsets $E$ such that, given $(x, y) \in E$, we can find $U \in \tau$ and $V \in \sigma$ with

$$
(x, y) \in U \times V \subseteq E .
$$


If $U \in \tau$, then, since $\pi_{X}$ is continuous $U \times Y=\pi_{X}^{-1}(U) \in \lambda$. Similarly, if $V \in \sigma$ then $X \times V \in \lambda$. Thus

$$
U \times V=U \times Y \cap X \times V \in \lambda .
$$

If $E \in \mu$ then, given $(x, y) \in E$, we can find $U_{(x, y)} \in \tau$ and $V_{(x, y)} \in \sigma$ such that

$$
(x, y) \in U_{(x, y)} \times V_{(x, y)} \subseteq E,
$$

We observe that

$$
E \subseteq \bigcup_{(x, y) \in E} U_{(x, y)} \times V_{(x, y)} \subseteq E
$$

so $E=\bigcup_{(x, y) \in E} U_{(x, y)} \times V_{(x, y)}$ and, since the union of open sets is open, $E \in \lambda$. Thus $\mu \subseteq \lambda$.

It is easy to check that $\mu$ is a topology as follows.

(a) $\varnothing \in \mu$ vacuously. If $(x, y) \in X \times Y$, then $X \in \tau, Y \in \sigma$ and $(x, y) \in X \times Y \subseteq X \times Y$. Thus $X \times Y \in \mu$.

(b) Suppose $E_{\alpha} \in \mu$ for all $\alpha \in A$. If $(x, y) \in \bigcup_{\alpha \in A} E_{\alpha}$, then $(x, y) \in E_{\beta}$ for some $\beta \in A$. We can find $U \in \tau$ and $V \in \sigma$ such that

$$
(x, y) \in U \times V \subseteq E_{\beta}
$$

and so

$$
(x, y) \in U \times V \subseteq \bigcup_{\alpha \in A} E_{\alpha}
$$

Thus $\bigcup_{\alpha \in A} E_{\alpha} \in \mu$.

(c) Suppose $E_{j} \in \mu$ for all $1 \leq j \leq n$. If $(x, y) \in \bigcap_{j=1}^{n} E_{j}$, then $(x, y) \in E_{j}$ for all $1 \leq j \leq n$. We can find $\bar{U}_{j} \in \tau$ and $V_{j} \in \sigma$ such that

$$
(x, y) \in U_{j} \times V_{j} \subseteq E_{j}
$$

and so

$$
(x, y) \in \bigcap_{j=1}^{n} U_{j} \times \bigcap_{j=1}^{n} V_{j} \subseteq \bigcap_{j=1}^{n} E_{j}
$$

Since $\bigcap_{j=1}^{n} U_{j} \in \tau$ and $\bigcap_{j=1}^{n} V_{j} \in \sigma$, we have shown that $\bigcap_{j=1}^{n} E_{j} \in \mu$.

Finally, we observe that, if $U \in \tau$, then

$$
\pi_{X}^{-1}(U)=U \times Y
$$

and $(x, y) \in U \times Y \subseteq \pi_{X}^{-1}(U)$ with $U \in \tau, Y \in \sigma$, so $\pi_{X}^{-1}(U) \in \mu$. Thus $\pi_{X}: X \times Y \rightarrow X$ is continuous if we give $X \times Y$ the topology $\mu$. A similar result holds for $\pi_{Y}$ so, by the minimality of $\lambda, \mu=\lambda$.

[Return to page 18.] 
Lemma 7.13. Let $\tau_{1}$ and $\tau_{2}$ be two topologies on the same space $X$.

(i) We have $\tau_{1} \subseteq \tau_{2}$ if and only if, given $x \in U \in \tau_{1}$, we can find $V \in \tau_{2}$ such that $x \in V \subseteq U$.

(ii) We have $\tau_{1}=\tau_{2}$ if and only if, given $x \in U \in \tau_{1}$, we can find $V \in \tau_{2}$ such that $x \in V \subseteq U$ and, given $x \in U \in \tau_{2}$, we can find $V \in \tau_{1}$ such that $x \in V \subseteq U$.

Proof. (i) If $\tau_{1} \subseteq \tau_{2}$ and $x \in U \in \tau_{1}$, then setting $V=U$ we automatically have $V \in \tau_{2}$ and $x \in V \subseteq U$.

Conversely, suppose that, given $x \in U \in \tau_{1}$, we can find $V \in \tau_{2}$ such that $x \in V \subseteq U$. Then, if $U \in \tau_{1}$ is fixed, we can find $V_{x} \in \tau_{2}$ such that $x \in V_{x} \subseteq U$ for each $x \in U$.

Now

$$
U \subseteq \bigcup_{x \in U} V_{x} \subseteq U
$$

so $U=\bigcup_{x \in U} V_{x}$ and, since the union of open sets is open, $U \in \tau_{2}$. Thus $\tau_{1} \subseteq \tau_{2}$.

(ii) Observe that $\tau_{1}=\tau_{2}$ if and only if $\tau_{1} \subseteq \tau_{2}$ and $\tau_{2} \subseteq \tau_{1}$.

[Return to page 19.]

Exercise 8.4. If $(X, \tau)$ is a topological space, then a subset $A$ of $X$ is open if and only if every point of $A$ has an open neighbourhood $U \subseteq A$.

Solution. If $A$ is open, then $A$ is an open neighbourhood of every $x \in A$.

Conversely, suppose that every $x \in A$ has an open neighbourhood $U_{x}$ lying entirely within $A$. Then

$$
A \subseteq \bigcup_{x \in A} U_{x} \subseteq A
$$

so $A=\bigcup_{x \in A} U_{x}$. Thus $A$ is the union of open sets and so open.

[Return to page 21.]

Lemma 8.5. If $(X, \tau)$ is a Hausdorff space, then the one point sets $\{x\}$ are closed.

Proof. We must show that $A=X \backslash\{x\}$ is open. But, if $y \in A$, then $y \neq x$ so, by the Hausdorff condition, we can find $U, V \in \tau$ such that $x \in U, y \in V$ and $U \cap V=\varnothing$. We see that $y \in V \subseteq A$, so every point of $A$ has an open neighbourhood lying entirely within $A$. Thus $A$ is open.

[Return to page 21.] 
Exercise 8.6. Let $X$ be infinite (we could take $X=\mathbb{Z}$ or $X=\mathbb{R}$ ). We say that a subset $E$ of $X$ lies in $\tau$ if either $E=\varnothing$ or $X \backslash E$ is finite. Show that $\tau$ is a topology and that every one point set $\{x\}$ is closed but that $(X, \tau)$ is not Hausdorff.

What happens if $X$ is finite?

Solution. (a) We are told that $\varnothing \in \tau$. Since $X \backslash X=\varnothing, X \in \tau$.

(b) If $U_{\alpha} \in \tau$ for all $\alpha \in A$, then either $U_{\alpha}=\varnothing$ for all $\alpha \in A$, so $\bigcup_{\alpha \in A} U_{\alpha}=\varnothing \in \tau$, or we can find a $\beta \in A$ such that $X \backslash U_{\beta}$ is finite. In the second case, we observe that

$$
X \backslash \bigcup_{\alpha \in A} U_{\alpha} \subseteq X \backslash U_{\beta}
$$

so $X \backslash \bigcup_{\alpha \in A} U_{\alpha}$ is finite and $\bigcup_{\alpha \in A} U_{\alpha} \in \tau$

(c) If $U_{j} \in \tau$ for all $1 \leq j \leq n$ then, either $U_{k}=\varnothing$ for some $1 \leq k \leq n$ so $\bigcap_{j=1}^{n} U_{j}=\varnothing \in \tau$ or $X \backslash U_{j}$ is finite for all $1 \leq j \leq n$. In the second case then, since

$$
X \backslash \bigcap_{j=1}^{n} U_{j}=\bigcup_{j=1}^{n}\left(X \backslash U_{j}\right),
$$

it follows that $X \backslash \bigcap_{j=1}^{n} U_{j}$ is finite and so $\bigcap_{j=1}^{n} U_{j} \in \tau$.

Thus $\tau$ is a topology.

Since $\{x\}$ is finite, $X \backslash\{x\}$ is open and so $\{x\}$ is closed.

Suppose that $x \neq y$ and $x \in U \in \tau, y \in V \in \tau$. Then $U, V \neq \varnothing$, so $X \backslash U$ and $X \backslash V$ is finite. It follows that

$$
X \backslash U \cap V=(X \backslash U) \cup(X \backslash V)
$$

is finite, and so, since $X$ is infinite, $U \cap V \neq \varnothing$. Thus $\tau$ is not Hausdorff.

If $X$ is finite, then $\tau$ is the discrete metric which is Hausdorff.

[Return to page 21.]

Lemma 8.8. If $(X, \tau)$ is a Hausdorff topological space and $Y \subseteq X$, then $Y$ with the subspace topology is also Hausdorff.

Proof. Write $\tau_{Y}$ for the subspace topology. If $x, y \in Y$ and $x \neq y$, then $x, y \in X$ and $x \neq y$ so we can find $U, V \in \tau$ with $x \in U, y \in V$ and $U \cap V=\varnothing$. Set $\tilde{U}=U \cap Y$ and $\tilde{V}=V \cap Y$. Then $\tilde{U}, \tilde{V} \in \tau_{Y} x \in \tilde{U}, y \in \tilde{V}$ and $\tilde{U} \cap \tilde{V}=\varnothing$.

[Return to page 22.]

Lemma 8.9. If $(X, \tau)$ and $(Y, \sigma)$ are Hausdorff topological spaces, then $X \times Y$ with the product topology is also Hausdorff. 
Proof. Suppose $\left(x_{1}, y_{1}\right),\left(x_{2}, y_{2}\right)$ and $\left(x_{1}, y_{1}\right) \neq\left(x_{2}, y_{2}\right)$. Then we know that at least one of the statements $x_{1} \neq x_{2}$ and $y_{1} \neq y_{2}$ is true ${ }^{12}$. Without loss of generality, we may suppose $x_{1} \neq x_{2}$. Since $(X, \tau)$ is Hausdorff, we can find $U_{1}, U_{2}$ disjoint open neighbourhoods of $x_{1}$ and $x_{2}$. We observe that $U_{1} \times Y$ and $U_{2} \times Y$ are disjoint open neighbourhoods of $\left(x_{1}, y_{1}\right)$ and $\left(x_{2}, y_{2}\right)$, so we are done.

[Return to page 22.]

Exercise 9.4. (iv) Show that the topology described in Exercise 8.6 is compact.

(v) Let $X$ be uncountable (we could take $X=\mathbb{R}$ ). We say that a subset $A$ of $X$ lies in $\tau$ if either $A=\varnothing$ or $X \backslash A$ is countable. Show that $\tau$ is a topology but that $(X, \tau)$ is not compact.

Solution. (iv) If $X=\varnothing$ there is nothing to prove. If not, let $U_{\alpha}[\alpha \in A]$ be an open cover. Since $X \neq \varnothing$ we can choose a $\beta \in A$ such that $U_{\beta} \neq \varnothing$ and so $U_{\beta}=X \backslash F$ where $F$ is a finite set. For each $x \in F$ we know that $x \in X=\bigcup_{\alpha \in A} U_{\alpha}$ so there exists an $\alpha(x) \in A$ with $x \in U_{\alpha(x)}$. We have

$$
U_{\beta} \cup \bigcup_{x \in F} U_{\alpha(x)}=X
$$

giving us the desired open cover.

(v) I leave it the reader to show that $\tau$ is a topology. Let $x_{1}, x_{2}, \ldots$, be distinct points of $X$. Let

$$
U=X \backslash\left\{x_{j}: 1 \leq j\right\}
$$

and $U_{k}=U \cup\left\{x_{k}\right\}$. Then $U_{k} \in \tau[k \geq 1]$ and $\bigcup_{k \geq 1} U_{k}=X$. Now suppose $k(1), k(2), \ldots, k(N)$ given. If $m=\max _{1 \leq r \leq N} k(r)$, then

$$
x_{m+1} \notin \bigcup_{r=1}^{N} U_{k(r)}
$$

so there is no finite subcover.

[Return to page 23.]

Theorem 9.5. [The Heine-Borel Theorem.] Let $\mathbb{R}$ be given its usual (Euclidean) topology. Then the closed bounded interval $[a, b]$ is compact.

\footnotetext{
${ }^{12}$ But not necessarily both. This is the traditional silly mistake.
} 
Proof. Suppose that $\mathcal{C}$ is an open cover of $[a, b]$ (i.e. the elements of $\mathcal{C}$ are open sets and $\left.\bigcup_{U \in \mathcal{C}} U \supseteq[a, b]\right)$. If $\mathcal{C}_{1}$ is a finite subcover of $[a, c]$ and $\mathcal{C}_{2}$ is a finite subcover of $[c, b]$ then $\mathcal{C}_{1} \cup \mathcal{C}_{2}$ is a finite subcover of $[a, b]$.

Suppose now that $[a, b]$ has no finite subcover using $\mathcal{C}$. Set $a_{0}=a, b_{0}=b$, and $c_{0}=\left(a_{0}+b_{0}\right) / 2$. By the first paragraph at least one of $\left[a_{0}, c_{0}\right]$ and $\left[c_{0}, b_{0}\right]$ has no finite subcover using $\mathcal{C}$. If $\left[a_{0}, c_{0}\right]$ has no finite subcover, set $a_{1}=a_{0}$, $b_{1}=c_{0}$. Otherwise, set $a_{1}=c_{0}, b_{1}=b_{0}$. In either case, we know that

(i) $a=a_{0} \leq a_{1} \leq b_{1} \leq b_{0}=b$,

(ii) if $\mathcal{F}$ is a finite subset of $\mathcal{C}$, then $\bigcup_{U \in \mathcal{F}} U \nsupseteq\left[a_{1}, b_{1}\right]$,

(iii) $b_{1}-a_{1}=(b-a) / 2$.

Proceeding inductively, we obtain

(i) ${ }_{n} a \leq a_{n-1} \leq a_{n} \leq b_{n} \leq b_{n-1} \leq b$.

(ii) ${ }_{n}$ If $\mathcal{F}$ is a finite subset of $\mathcal{C}$, then $\bigcup_{U \in \mathcal{F}} U \nsupseteq\left[a_{n}, b_{n}\right]$.

(iii) ${ }_{n} b_{n}-a_{n}=2^{-n}(b-a)$.

The $a_{n}$ form an increasing sequence bounded above by $b$, so, by the fundamental axiom of analysis, $a_{n} \rightarrow A$ for some $A \leq b$. Similarly $b_{n} \rightarrow B$ for some $B \geq a$. Since $b_{n}-a_{n} \rightarrow 0, A=B=x$, say, for some $x \in[a, b]$. Since $x \in[a, b]$ and $\bigcup_{U \in \mathcal{C}} U \supseteq[a, b]$ we can find a $V \in \mathcal{C}$ with $x \in V$. Since $V$ is open in the Euclidean metric, we can find a $\delta>0$ such that $(x-\delta, x+\delta) \subseteq V$. Since $a_{n}, b_{n} \rightarrow x$ we can find an $N$ such that $\left|x-a_{N}\right|,\left|x-b_{N}\right|<\delta$ and so

$$
\left[a_{N}, b_{N}\right] \subseteq(x-\delta, x+\delta) \subseteq V
$$

contradicting $(\mathrm{ii})_{N}$. (Just take $\mathcal{F}=\{V\}$.)

The theorem follows by reductio ad absurdum.

[Return to page 23.]

Theorem 9.7. A closed subset of a compact set is compact. [More precisely, if $E$ is compact and $F$ closed in a given topology, then, if $F \subseteq E$, it follows that $F$ is compact.]

Proof. Suppose $(X, \tau)$ is a topological space, $E$ is a compact set in $X$ and $F$ is a closed subset of $E$. If $U_{\alpha} \in \tau[\alpha \in A]$ and $\bigcup_{\alpha \in A} U_{\alpha} \supseteq F$, then $X \backslash F \in \tau$ and

$$
(X \backslash F) \cup \bigcup_{\alpha \in A} U_{\alpha}=X \supseteq E .
$$

By compactness, we can find $\alpha(j) \in A[1 \leq j \leq n]$ such that

$$
(X \backslash F) \cup \bigcup_{j=1}^{n} U_{\alpha(j)} \supseteq E .
$$


Since $(X \backslash F) \cap F=\varnothing$ and $E \supseteq F$, it follows that

$$
\bigcup_{j=1}^{n} U_{\alpha(j)} \supseteq F
$$

and we are done. [Return to page 24.]

Theorem9.8. If $(X, \tau)$ is Hausdorff, then every compact set is closed.

Proof. Let $K$ be a compact set. If $x \notin K$, then, given any $k \in K$, we know that $k \neq x$ and so, since $X$ is Hausdorff, we can find open sets $U_{k}$ and $V_{k}$ such that

$$
x \in V_{k}, k \in U_{k} \text { and } V_{k} \cap U_{k}=\varnothing .
$$

Since $\bigcup_{k \in K} U_{k} \supseteq \bigcup_{k \in K}\{k\}=K$, we have an open cover of $K$. By compactness, we can find $k(1), k(2), \ldots, k(n) \in K$ such that

$$
\bigcup_{j=1}^{n} U_{k(j)} \supseteq K
$$

We observe that the finite intersection $V=\bigcap_{j=1}^{n} V_{k(j)}$ is an open neighbourhood of $x$ and that

$$
V \cap K \subseteq V \cap \bigcup_{j=1}^{n} U_{k(j)}=\varnothing,
$$

so $V \cap K$ and we have shown that every $x \in X \backslash K$ has an open neighbourhood lying entirely within $X \backslash K$. Thus $X \backslash K$ is open and $K$ is closed.

[Return to page 24.]

Example 9.9. Give an example of a topological space and a compact set which is not closed.

Proof. If $(X, \tau)$ has the indiscrete topology, then, if $Y \subseteq X, Y \neq X, \varnothing$, we have $Y$ compact but not closed. We can take $X=\{a, b\}$ with $a \neq b$ and $Y=\{a\}$.

[Return to page 24.]

Theorem 9.10. Consider $(\mathbb{R}, \tau)$ with the standard (Euclidean) topology. A set $E$ is compact if and only if it is closed and bounded (that is to say, there exists a $M$ such that $|x| \leq M$ for all $x \in E$ ). 
Proof. If $E$ is bounded, then $E \subseteq[-M, M]$ for some $M$. By the theorem of Heine-Borel, $[-M, M]$ is compact so, if $E$ is closed, $E$ is compact.

Since $(\mathbb{R}, \tau)$ is Hausdorff any compact set must be closed. Finally suppose that $E$ is compact. We have

$$
E \subseteq \bigcup_{j=1}^{\infty}(-j, j)
$$

By compactness, we can find $j(r)$ such that $E \subseteq \bigcup_{r=1}^{N}(-j(r), j(r))$ Taking $M=\max _{1 \leq r \leq n} j(r)$ we have $E \subseteq(-M, M)$ so $E$ is bounded.

[Return to page 24.]

Theorem 9.12. Let $(X, \tau)$ and $(Y, \sigma)$ be topological spaces and $f: X \rightarrow Y$ a continuous function. If $K$ is a compact subset of $X$, then $f(K)$ is a compact subset of $Y$.

Proof. Suppose that $U_{\alpha} \in \sigma$ for all $\alpha \in A$ and $\bigcup_{\alpha \in A} U_{\alpha} \supseteq f(K)$. Then

$$
\bigcup_{\alpha \in A} f^{-1}\left(U_{\alpha}\right)=f^{-1}\left(\bigcup_{\alpha \in A} U_{\alpha}\right) \supseteq K
$$

and, since $f$ is continuous $f^{-1}\left(U_{\alpha}\right) \in \tau$ for all $\alpha \in A$. By compactness, we can find $\alpha(j) \in A[1 \leq j \leq n]$ such that

$$
\bigcup_{j=1}^{n} f^{-1}\left(U_{\alpha(j)}\right) \supseteq K
$$

and so

$$
\bigcup_{j=1}^{n} U_{\alpha(j)}=f\left(\bigcup_{j=1}^{n} f^{-1}\left(U_{\alpha(j)}\right)\right) \supseteq f(K)
$$

and we are done.

[Return to page 24.]

Theorem 9.15. Let $\mathbb{R}$ have the usual metric. If $K$ is a closed and bounded subset of $\mathbb{R}$ and $f: K \rightarrow \mathbb{R}$ is continuous, then $f$ is bounded and attains its bounds.

Proof. If $K$ is empty there is nothing to prove, so we assume $K \neq \varnothing$.

Since $K$ is compact and $f$ is continuous, $f(K)$ is compact. Thus $f(K)$ is a non-empty closed bounded set. Since $f(K)$ is non-empty and bounded, 
it has a supremum $\alpha$, say. Since $f(K)$ is closed, it contains its supremum. [Observe that we can find $k_{n} \in K$ such that

$$
\alpha-1 / n \leq f\left(k_{n}\right) \leq \alpha
$$

and so $f\left(k_{n}\right) \rightarrow \alpha$. Since $f(K)$ is closed, $\alpha \in f(K)$.]

[Return to page 25.]

Exercise 9.16. Let $\mathbb{R}$ have the usual metric. If $K$ is a subset of $\mathbb{R}$ with the property that, whenever $f: K \rightarrow \mathbb{R}$ is continuous, then $f$ is bounded, it follows that $K$ is closed and bounded.

Proof. If $K=\varnothing$ there is nothing to prove, so we assume $K \neq \varnothing$.

Let $f: K \rightarrow \mathbb{R}$ be defined by $f(k)=|k|$. Since $f$ is bounded, $K$ must be.

If $x \notin K$, then the function $f: K \rightarrow \mathbb{R}$ given by $f(k)=|k-x|^{-1}$ is continuous and so bounded. Thus we can find an $M>0$ such that $|f(k)|<$ $M$ for all $k \in K$. It follows that $|x-k|>M^{-1}$ for all $k \in K$ and the open ball $B\left(x, M^{-1}\right)$ lies entirely in the complement of $K$. Thus $K$ is closed.

[Return to page 25.]

Theorem 9.18. Let $(X, \tau)$ be a compact and $(Y, \sigma)$ a Hausdorff topological space. If $f: X \rightarrow Y$ is a continuous bijection, then it is a homeomorphism.

Proof. Since $f$ is a bijection, $g=f^{-1}$ is a well defined function. If $K$ is closed in $X$, then (since a closed subset of a compact space is compact) $K$ is compact so $f(K)$ is compact. But a compact subset of a Hausdorff space is closed so $g^{-1}(K)=f(K)$ is closed. Thus $g$ is continuous and we are done. (If $U$ is open in $X$ then $X \backslash U$ is closed so $Y \backslash g^{-1}(U)=g^{-1}(X \backslash U)$ is closed and $g^{-1}(U)$ is open.)

[Return to page 25.]

Theorem 9.20. Let $\tau_{1}$ and $\tau_{2}$ be topologies on the same space $X$.

(i) If $\tau_{1} \supseteq \tau_{2}$ and $\tau_{1}$ is compact, then so is $\tau_{2}$.

(ii) If $\tau_{1} \supseteq \tau_{2}$ and $\tau_{2}$ is Hausdorff, then so is $\tau_{1}$.

(iii) If $\tau_{1} \supseteq \tau_{2}, \tau_{1}$ is compact and $\tau_{2}$ is Hausdorff, then $\tau_{1}=\tau_{2}$.

Proof. (i) The map $\iota:\left(X, \tau_{1}\right) \rightarrow\left(X, \tau_{2}\right)$ is continuous and so takes compact sets to compact sets. In particular, since $X$ is compact, in $\tau_{1}, X=\iota X$ is compact in $\tau_{2}$.

(ii) If $x \neq y$ we can find $x \in U \in \tau_{2}$ and $y \in V \in \tau_{2}$ with $U \cap V=\varnothing$. Automatically $x \in U \in \tau_{1}$ and $y \in V \in \tau_{1}$ so we are done.

(iii) The map $\iota:\left(X, \tau_{1}\right) \rightarrow\left(X, \tau_{2}\right)$ is a continuous bijection and so a homeomorphism.

[Return to page 26.] 
Exercise 9.21. (i) Give an example of a Hausdorff space $(X, \tau)$ and a compact Hausdorff space $(Y, \sigma)$ together with a continuous bijection $f: X \rightarrow Y$ which is not a homeomorphism.

(ii) Give an example of a compact Hausdorff space $(X, \tau)$ and a compact space $(Y, \sigma)$ together with a continuous bijection $f: X \rightarrow Y$ which is not a homeomorphism.

Solution. Let $\tau_{1}$ be the indiscrete topology on $[0,1], \tau_{2}$ be the usual (Euclidean) topology on $[0,1]$ and $\tau_{3}$ be the discrete topology on $[0,1]$. Then ( $\left.\mathbb{R}, \tau_{1}\right)$ is compact (but not Hausdorff), ( $\left.\mathbb{R}, \tau_{2}\right)$ is compact and Hausdorff, and $\left(\mathbb{R}, \tau_{3}\right)$ is Hausdorff (but not compact). The identity maps $\iota:\left(X, \tau_{1}\right) \rightarrow$ $\left(X, \tau_{2}\right)$ and $\iota:\left(X, \tau_{2}\right) \rightarrow\left(X, \tau_{3}\right)$ are continuous bijections but not homeomorphisms.

[Return to page 26.]

Theorem 10.1. The product of two compact spaces is compact. (More formally, if $(X, \tau)$ and $(Y, \sigma)$ are compact topological spaces and $\lambda$ is the product topology, then $(X \times Y, \lambda)$ is compact.)

Proof. Let $O_{\alpha} \in \lambda[\alpha \in A]$ and

$$
\bigcup_{\alpha \in A} O_{\alpha}=X \times Y
$$

Then, given $(x, y) \in X \times Y$, we can find $U_{x, y} \in \tau, V_{x, y} \in \sigma$ and $\alpha(x, y) \in A$ such that

$$
(x, y) \in U_{x, y} \times V_{x, y} \subseteq O_{\alpha(x, y)} .
$$

In particular, we have

$$
\bigcup_{y \in Y}\{x\} \times V_{x, y}=\{(x, y): y \in Y\}
$$

for each $x \in X$ and so

$$
\bigcup_{y \in Y} V_{x, y}=Y .
$$

By compactness, we can find a positive integer $n(x)$ and $y(x, j) \in Y[1 \leq$ $j \leq n(x)$ ] such that

$$
\bigcup_{j=1}^{n(x)} V_{x, y(x, j)}=Y .
$$


Now $U_{x}=\bigcap_{j=1}^{n(x)} U_{x, y(x, j)}$ is the finite intersection of open sets in $X$ and so open. Further $x \in U_{x}$ and so

$$
\bigcup_{x \in X} U_{x}=X
$$

By compactness, we can find $x_{1}, x_{2}, \ldots, x_{m}$ such that

$$
\bigcup_{r=1}^{m} U_{x_{r}}=X \text {. }
$$

It follows that

$$
\begin{aligned}
\bigcup_{r=1}^{m} \bigcup_{j=1}^{n\left(x_{r}\right)} O_{x_{r}, y\left(x_{r}, j\right)} & \supseteq \bigcup_{r=1}^{m} \bigcup_{j=1}^{n\left(x_{r}\right)} U_{x_{r}, y\left(x_{r}, j\right)} \times V_{x_{r}, y\left(x_{r}, j\right)} \\
& \supseteq \bigcup_{r=1}^{m} \bigcup_{j=1}^{n\left(x_{r}\right)} U_{x_{r}} \times V_{x_{r}, y\left(x_{r}, j\right)} \\
& \supseteq \bigcup_{r=1}^{m} U_{x_{r}} \times Y \\
& \supseteq X \times Y
\end{aligned}
$$

and we are done.

[Return to page 26.]

Exercise 10.7. Consider the complex plane with its usual metric. Let

$$
\partial D=\{z \in \mathbb{C}:|z|=1\}
$$

and give $\partial D$ the subspace topology $\tau$. Give $\mathbb{R}$ its usual topology and define an equivalence relation $\sim$ by $x \sim y$ if $x-y \in \mathbb{Z}$. We write $\mathbb{R} / \sim=\mathbb{T}$ and give $\mathbb{T}$ the quotient topology. The object of this exercise is to show that $\partial D$ and $\mathbb{T}$ are homeomorphic.

(i) Verify that $\sim$ is indeed an equivalence relation.

(ii) Show that, if we define $f: \mathbb{R} \rightarrow \partial D$ by $f(x)=\exp (2 \pi i x)$, then $f(U)$ is open whenever $U$ is open.

(iii) If $q: \mathbb{R} \rightarrow \mathbb{T}$ is the quotient map $q(x)=[x]$ show that $q(x)=q(y)$ if and only if $f(x)=f(y)$. Deduce that $q\left(f^{-1}(\{\exp (2 \pi i x)\})\right)=[x]$ and that the equation $F(\exp (2 \pi i x))=[x]$ gives a well defined bijection $F: \partial D \rightarrow \mathbb{T}$.

(iv) Show that $F^{-1}(V)=f\left(q^{-1}(V)\right)$ and deduce that $F$ is continuous.

(v) Show that $\mathbb{T}$ is Hausdorff and explain why $\partial D$ is compact. Deduce that $F$ is a homeomorphism. 
Solution. (i) Observe that $x-x=0 \in \mathbb{Z}$ so $x \sim x$.

Observe that $x \sim y$ implies $x-y \in \mathbb{Z}$ so $y-x=-(x-y) \in \mathbb{Z}$ and $y \sim x$.

Observe that, if $x \sim y$ and $y \sim z$, then $x-y, y-z \in \mathbb{Z}$ so

$$
x-z=(x-y)+(y-z)=x-z \in \mathbb{Z}
$$

and $x \sim z$.

(ii) If $x \in U$ an open set, then we can find a $1>\delta>0$ such that $|x-y|<\delta$ implies $y \in U$.

By simple geometry, any $z \in \mathbb{C}$ with $|z|=1$ and $|\exp (2 \pi i x)-z|<\delta / 100$ can be written as $z=\exp (2 \pi i y)$ with $|y-x|<\delta$. Thus

$$
\partial D \cap\{z \in \mathbb{C}:|z-\exp (2 \pi i x)|<\delta / 100\} \subseteq f(U) .
$$

We have shown that $f(U)$ is open.

(iii) We have

$$
\begin{aligned}
q(x)=q(y) & \Leftrightarrow y \in[x] \Leftrightarrow x-y \in \mathbb{Z} \Leftrightarrow \exp (2 \pi i(x-y))=1 \\
& \Leftrightarrow \exp (2 \pi i x)=\exp (2 \pi i y) \Leftrightarrow f(x)=f(y) .
\end{aligned}
$$

It follows that the equation $F(\exp (2 \pi i x))=[x]$ gives a well defined bijection $F: \partial D \rightarrow \mathbb{T}$.

(iv) Observe that

$$
F^{-1}([x])=\{\exp (2 \pi i t): \exp (2 \pi i t)=\exp (2 \pi i x)\}=f\left(q^{-1}([x])\right)
$$

and so $F^{-1}(V)=f\left(q^{-1}(V)\right)$. If $V$ is open, then, since $q$ is continuous, $q^{-1}(V)$ is open so, by (ii), $F^{-1}(V)$ is open. Thus $F$ is continuous.

(v) If $[x] \neq[y]$, then we know that $x-y \notin \mathbb{Z}$ and the set

$$
\{|t|: t-(x-y) \in \mathbb{Z},|t|<1\}
$$

is finite and non-empty. Thus there exists a $\delta>0$ such that

$$
\{|t|: t-(x-y) \in \mathbb{Z},|t|<\delta\}=\{\varnothing\} .
$$

Let

$$
U_{x}=\bigcup_{j=-\infty}^{\infty}(j+x-\delta / 4, j+x+\delta / 4) \text { and } U_{y}=\bigcup_{j=-\infty}^{\infty}(j+y-\delta / 4, j+y+\delta / 4) .
$$

Observe that $U_{x}$ and $U_{y}$ are open in $\mathbb{R}$ and $q^{-1}\left(q\left(U_{x}\right)\right)=U_{x}, q^{-1}\left(q\left(U_{y}\right)\right)=U_{y}$, and so $q\left(U_{x}\right)$ and $q\left(U_{y}\right)$ are open in the quotient topology. Since $[x] \in q\left(U_{x}\right)$, 
$[y] \in q\left(U_{y}\right)$ and $q\left(U_{x}\right) \cap q\left(U_{y}\right)=\varnothing$, we have shown that the quotient topology is Hausdorff.

Since $\partial D$ is closed and bounded in $\mathbb{C}$ and we can identify $\mathbb{C}$ with $\mathbb{R}^{2}$ as a metric space, $\partial D$ is compact.

Since a continuous bijection from a compact to a Hausdorff space is a homeomorphism, $F$ is a homeomorphism.

[Remark. It is just as simple to show that the natural map from $\mathbb{T}$ (which we know to be compact, why?) to $\partial D$ (which we know to be Hausdorff, why?) is a bijective continuous map. Or we could show continuity in both directions and not use the result on continuous bijections.]

[Return to page 28.]

Theorem 11.4. If $E$ is a subset of a topological space $(X, \tau)$, then $E$ is disconnected if and only if we can find a non-constant continuous function $f: E \rightarrow \mathbb{R}$ which only takes the value 0 or 1 .

Proof. Since we are dealing with a subspace topology, we can take $E=X$.

If $f: X \rightarrow \mathbb{R}$ is a continuous non-constant function which only takes the value 0 or 1 , then $U=f^{-1}(\{0\})=f^{-1}((-1 / 2,1 / 2))$ is open and non-empty and similarly $V=f^{-1}(\{1\})$ is. Since $V \cup U=X$ and $V \cap U=\varnothing$, it follows that $X$ is disconnected.

Conversely, if $X$ is disconnected, we can find non-empty open sets $U$ and $V$ such that $V \cup U=X$ and $V \cap U=\varnothing$. If we set $f(u)=0$ when $u \in U$ and $f(v)=1$ when $v \in V$, then $f: X \rightarrow \mathbb{R}$ is a well defined non-constant function which only takes the value 0 or 1 . If $A \subset \mathbb{R}$, the $f^{-1}(A)$ must be one of the four sets $\varnothing, U, V$ or $X$, all of which are open. Thus $f$ is continuous.

[Return to page 29.]

Theorem 11.5. If we give $\mathbb{R}$ the usual topology, then $\mathbb{R}$ and the intervals $[a, b]$ and $(a, b)$ are connected.

Proof. We prove the result for $(a, b)$. The other results are proved similarly.

Suppose $f:(a, b) \rightarrow \mathbb{R}$ is continuous and there exist $c, d \in(a, b)$ with $f(c)=0$ and $f(d)=1$. Without loss of generality we may suppose that $c<d$ and so $a<c<d<b$. By the intermediate value theorem, we can find $\gamma \in(c, d)$ with $f(\gamma)=1 / 2$. Since $\gamma \in(a, b), f$ takes at least three values and $(a, b)$ must be connected.

[Return to page 29.]

Exercise 11.6. (i) If $(X, \tau)$ and $(Y, \sigma)$ are topological spaces, $E$ is a connected subset of $X$ and $g: E \rightarrow Y$ is continuous, then $g(E)$ is connected. (More briefly, the continuous image of a connected set is connected.) 
(ii) If $(X, \tau)$ is a connected topological space and $\sim$ is an equivalence relation on $X$, then $X / \sim$ with the quotient topology is connected.

(iii) If $(X, \tau)$ and $(Y, \sigma)$ are connected topological spaces, then $X \times Y$ with the product topology is connected.

(iv) If $(X, \tau)$ is a connected topological space and $E$ is a subset of $X$, it does not follow that $E$ with the subspace topology is connected.

Proof. (i) If $g(E)$ is not connected we can find a non-constant continuous $f$ : $g(E) \rightarrow \mathbb{R}$ taking only the values 0 and 1 . Setting $F=f \circ g$ (the composition of $f$ and $g$ ), we know that $F: E \rightarrow \mathbb{R}$ is non-constant, continuous and only takes the values 0 and 1 . Thus $E$ is not connected.

(ii) $X / \sim$ is the continuous image of $X$ under the quotient map which we know to be continuous.

(iii) Suppose $X \times Y$ with the product topology is not connected. Then we can find a non-constant continuous function $f: X \times Y \rightarrow \mathbb{R}$ taking only the values 0 and 1 . Take $(x, y),(u, v) \in X \times Y$ with $f(x, y) \neq f(u, v)$. Then, if $f(x, v)=f(x, y)$, it follows that $f(x, v) \neq f(u, v)$. Without loss of generality, suppose that $f(x, v) \neq f(x, y)$. Then we know that the function $\theta: Y \rightarrow X \times Y$ given by $\theta(z)=(x, z)$ is continuous. (If $\Omega$ is open in $X \times Y$ and $z \in \theta^{-1}(\Omega)$, then $(x, z) \in \Omega$, so we can find $U$ open in $X$ and $V$ open in $Y$ such that $(x, z) \in U \times V \subseteq \Omega$. Thus $z \in V \subseteq \theta^{-1}(\Omega)$ and we have shown $\theta^{-1}(\Omega)$ open.) If we set $F=f \circ \theta$, then $F: Y \rightarrow \mathbb{R}$ is non-constant, continuous and only takes the values 0 and 1 . Thus $Y$ is not connected.

(iv) $\mathbb{R}$ is connected with the usual topology but $E=(-2,-1) \cup(1,2)$ is not.

[Return to page 29.]

Example 11.7. Suppose that $E$ is a connected subset of a topological space $(X, \tau)$. Suppose that $f: E \rightarrow \mathbb{R}$ is 'locally constant' in the sense that, given any $e \in E$, we can find an open neighbourhood $U$ of e such that $f$ is constant on $U \cap E$. Then $f$ is constant.

Proof. Since we are dealing with the subspace topology on $E$, there is no loss in generality in taking $E=X$. If $X=\varnothing$ the result is vacuous so we may take $X \neq \varnothing$.

Choose an $x_{0} \in X$ and set $c=f\left(x_{0}\right)$. Now consider the sets

$$
U=\{x \in X: f(x)=c\} \text { and } V=\{x \in X: f(x) \neq c\} .
$$

We claim that $U$ and $V$ are open. For suppose $v \in V$. Then we can find an open neighbourhood $N$ of $v$ such that $f$ is constant on $N$. Thus $f(x)=$ $f(v) \neq c$ for all $x \in N$, so $N \subseteq V$. It follows that $V$ is open. A similar, slightly simpler, argument shows that $U$ is open. 
Since $U \cap V=\varnothing, U \cup V=X$ and $U \neq \varnothing$ the connectedness of $X$ tells us that $V=\varnothing$ and $U=X$. The result follows.

[Return to page 30.]

Lemma 11.9. We work in a topological space $(X, \tau)$.

(i) Let $x_{0} \in X$. If $x_{0} \in E_{\alpha}$ and $E_{\alpha}$ is connected for all $\alpha \in A$, then $\bigcup_{\alpha \in A} E_{\alpha}$ is connected.

(ii) Write $x \sim y$ if there exists a connected set $E$ with $x, y \in E$. Then $\sim$ is an equivalence relation.

(iii) The equivalence classes $[x]$ are connected.

(iv) If $F$ is connected and $F \supseteq[x]$, then $F=[x]$.

Proof. (i) Let $U$ and $V$ be open sets such that

$$
U \cup V \supseteq \bigcup_{\alpha \in A} E_{\alpha} \text { and } U \cap V \cap \bigcup_{\alpha \in A} E_{\alpha}=\varnothing
$$

Without loss of generality, let $x_{0} \in U$. Then

$$
U \cup V \supseteq E_{\alpha} \text { and } U \cap V \cap E_{\alpha}=\varnothing
$$

for each $\alpha \in A$. But $x_{0} \in U \cap E_{\alpha}$ so $U \cap E_{\alpha} \neq \varnothing$, and so, by the connectedness of $E_{\alpha}$, we have

$$
U \supseteq E_{\alpha}
$$

for all $\alpha \in A$. Thus $U \supseteq \bigcup_{\alpha \in A} E_{\alpha}$. We have shown that $\bigcup_{\alpha \in A} E_{\alpha}$ is connected.

(ii) Observe that if $U$ and $V$ are sets (open or not) such that

$$
U \cup V \supseteq\{x\} \text {, and } U \cap V \cap\{x\}=\varnothing .
$$

then either $x \notin U$ and $U \cap\{x\}=\varnothing$ or $x \in U$ so $U \supseteq\{x\}$. Thus the one point set $\{x\}$ is connected and $x \sim x$.

The symmetry of the definition tells us that, if $x \sim y$, then $y \sim x$.

If $x \sim y$ and $y \sim z$, then $x, y \in E$ and $y, z \in F$ for some connected sets $E$ and $F$. By part (i), $E \cup F$ is connected (observe that $y \in E, F$ ) so, since $x, z \in E \cup F, x \sim z$.

We have shown that $\sim$ is an equivalence relation.

(iii) If $y \in[x]$, then there exists a connected set $E_{y}$ with $x, y \in E_{y}$. By definition $[x] \supseteq E_{y}$ so

$$
[x]=\bigcup_{y \in[x]}\{y\} \subseteq \bigcup_{y \in[x]} E_{y} \subseteq[x]
$$


whence

$$
[x]=\bigcup_{y \in[x]} E_{y}
$$

and, by part (i), $[x]$ is connected.

(iv) If $F$ is connected and $[x] \subseteq F$, then $x \in F$ and, by definition of $\sim$, $[x] \supseteq F$. It follows that $F=[x]$.

[Return to page 30.]

Lemma 11.11. If $(X, \tau)$ is a topological space and we write $x \sim y$ if $x$ is path-connected to $y$, then $\sim$ is an equivalence relation.

Proof. If $x \in X$, then the map $\gamma:[0,1] \rightarrow X$ defined by $\gamma(t)=x$ for all $t$ is continuous. (Observe that, if $F$ is a closed set in $\mathrm{X}$, then $\gamma^{-1}(F)$ takes the value $\varnothing$ or $[0,1]$ both of which are closed.) Thus $x \sim x$.

If $x \sim y$, then we can find a continuous map $\gamma:[0,1] \rightarrow X$ with $\gamma(0)=x$ and $\gamma(1)=y$. The map $T:[0,1] \rightarrow[0,1]$ given by $T(t)=1-t$ is continuous so the composition $\tilde{\gamma}=\gamma \circ T$ is. Observe that $\tilde{\gamma}(0)=y$ and $\tilde{\gamma}(1)=x$ so $y \sim x$.

If $x \sim y$ and $y \sim z$, then we can find continuous maps $\gamma_{j}:[0,1] \rightarrow X$ with $\gamma_{1}(0)=x, \gamma_{1}(1)=y, \gamma_{2}(0)=y$ and $\gamma_{2}(1)=z$. Define $\gamma:[0,1] \rightarrow X$ by

$$
\gamma(t)= \begin{cases}\gamma_{1}(2 t) & \text { if } t \in[0,1 / 2] \\ \gamma_{2}(2 t-1) & \text { if } t \in(1 / 2,1]\end{cases}
$$

If $U$ is open in $X$, then

$$
\gamma^{-1}(U)=\left\{t / 2: t \in \gamma_{1}^{-1}(U)\right\} \cup\left\{(1+t) / 2: t \in \gamma_{2}^{-1}(U)\right\}
$$

is open.

(If more detail is required we argue as follows. Suppose $s \in \gamma^{-1}(U)$. If $s \in(0,1 / 2)$, then $2 s \in \gamma_{1}^{-1}(U)$ so, since $\gamma_{1}^{-1}(U)$ is open we can find a $\delta>0$ with $s>\delta$ such that $(2 s-\delta, 2 s+\delta) \subseteq \gamma_{1}^{-1}(U)$. Thus $(s-\delta / 2, s+\delta / 2) \subseteq$ $\gamma^{-1}(U)$. If $s=0$ then $0 \in \gamma_{1}^{-1}(U)$ so, since $\gamma_{1}^{-1}(U)$ is open we can find a $\delta>0$ with $1>\delta$ such that $[0, \delta) \subseteq \gamma_{1}^{-1}(U)$. Thus $[s, \delta / 2)=[0, \delta / 2) \subseteq \gamma^{-1}(U)$. The cases $s \in(1 / 2,1]$ are dealt with similarly. This leaves the case $s=1 / 2$. Arguing as before, we can find $\delta_{1}, \delta_{2}>0$ with $1>\delta_{1}, \delta_{2}$ such that

$$
\left(1-\delta_{1}, 1\right] \subseteq \gamma_{1}^{-1}(U) \text { and }\left[0, \delta_{2}\right) \subseteq \gamma_{2}^{-1}(U) .
$$

Setting $\delta=\min \left(\delta_{1}, \delta_{2}\right)$ we have

$$
(s-\delta / 2, s+\delta / 2)=(1 / 2-\delta / 2,1 / 2+\delta / 2) \gamma^{-1}(U) \text {. }
$$


We see that the case $s=1 / 2$ is really the only one which requires care.)

Thus $\gamma$ is continuous and, since $\gamma(0)=x, \gamma(1)=z, x \sim z$.

[Return to page 85.]

Theorem 11.12. If a topological space is path-connected, then it is connected.

Proof. Suppose that $(X, \tau)$ is path-connected and that $U$ and $V$ are open sets with $U \cap V=\varnothing$ and $U \cup V=X$. If $U \neq \varnothing$, choose $x \in U$. If $y \in X$, we can find $f:[0,1] \rightarrow X$ continuous with $f(0)=x$ and $f(1)=y$. Now the continuous image of a connected set is connected and $[0,1]$ is connected, so $f([0,1])$ is connected. Since

$$
U \cap V \cap f([0,1])=\varnothing, U \cup V \supseteq f([0,1]) \text { and } U \cap f([0,1]) \neq \varnothing,
$$

we know that $U \supseteq f([0,1])$ so $y \in U$. Thus $U=X$. We have shown that $X$ is connected.

[Return to page 31.]

Theorem 11.13. If we give $\mathbb{R}^{n}$ the usual topology, then any open set $\Omega$ which is connected is path-connected.

Proof. If $\Omega=\varnothing$, there is nothing to prove, so we assume $\Omega$ non-empty.

Pick $\mathbf{x} \in \Omega$ and let $U$ be the set of all points in $\Omega$ which are path-connected to $\mathbf{x}$ and let $V$ be the set of all points in $\Omega$ which are not. We shall prove that $U$ and $V$ are open.

Suppose first that $\mathbf{u} \in U$. Since $\Omega$ is open, we can find an open ball $B(\mathbf{u}, \delta)$ centre $\mathbf{u}$, radius $\delta>0$ lying entirely within $\Omega$. If $\mathbf{y} \in B(\mathbf{u}, \delta)$, then $\mathbf{u}$ is path-connected to $\mathbf{y}$ in $B(\mathbf{u}, \delta)$ and so in $U$. (Consider $\gamma:[0,1] \rightarrow \Omega$ given by $\gamma(t)=t \mathbf{u}+(1-t) \mathbf{y}$.) Since $\mathbf{x}$ is path-connected to $\mathbf{u}$ and $\mathbf{u}$ is path-connected to $\mathbf{y}$, it follows that $\mathbf{x}$ is path-connected to $\mathbf{y}$ in $\Omega$ so $\mathbf{y} \in U$.

Now suppose that $\mathbf{v} \in V$. Since $\Omega$ is open, we can find an open ball $B(\mathbf{v}, \delta)$ centre $\mathbf{v}$, radius $\delta>0$ lying entirely within $\Omega$. If $\mathbf{y} \in B(\mathbf{v}, \delta)$, then $\mathbf{v}$ is path-connected to $\mathbf{y}$ in $B(\mathbf{v}, \delta)$ and so in $V$. It follows that, if $\mathbf{y}$ is path-connected to $\mathbf{x}$, then so is $\mathbf{v}$. But $\mathbf{v} \in V$, so $\mathbf{y}$ is not path-connected to $\mathbf{x}$. Thus $\mathbf{y} \in V$.

Since $U \cup V=\Omega$ and $U \cap V=\varnothing$, the connectedness of $\Omega$ shows that $U=\Omega$ and $\Omega$ is path-connected.

[Return to page 31.]

Example 11.14. We work in $\mathbb{R}^{2}$ with the usual topology. Let

$$
E_{1}=\{(0, y):|y| \leq 1\} \text { and } E_{2}=\{(x, \sin 1 / x): 0<x \leq 1\}
$$


and set $E=E_{1} \cup E_{2}$.

(i) Sketch E.

(ii) Explain why $E_{1}$ and $E_{2}$ are path-connected and show that $E$ is closed and connected.

(iii) Suppose, if possible, that $\mathbf{x}:[0,1] \rightarrow E$ is continuous and $\mathbf{x}(0)=$ $(1,0), \mathbf{x}(1)=(0,0)$. Explain why we can find $0<t_{1}<t_{2}<t_{3}<\ldots$ such that $x\left(t_{j}\right)=\left(\left(j+\frac{1}{2}\right) \pi\right)^{-1}$. By considering the behaviour of $t_{j}$ and $y\left(t_{j}\right)$, obtain a contradiction.

(iv) Deduce that $E$ is not path-connected.

Solution. Part (i) is left to the reader.

(ii) If $y_{1}, y_{2} \in[-1,1]$, the function $\mathbf{f}:[0,1] \rightarrow E_{1}$ given by

$$
\mathbf{f}(t)=\left(0,(1-t) y_{1}+t y_{2}\right)
$$

is continuous and $\mathbf{f}(0)=\left(x_{1}, 0\right)$ and $\mathbf{f}(1)=\left(x_{2}, 0\right)$, so $E_{1}$ is path-connected.

If $\left(x_{1}, y_{1}\right),\left(x_{2}, y_{2}\right) \in E_{2}$, then $y_{j}=\sin 1 / x_{j}$ and setting

$$
\mathbf{g}(t)=\left((1-t) x_{1}+t x_{2}, \sin \left(1 /\left((1-t) x_{1}+t x_{2}\right)\right)\right)
$$

we see that $\mathbf{g}$ is continuous and $\mathbf{g}(0)=\left(x_{1}, y_{1}\right)$ and $\mathbf{g}(1)=\left(x_{2}, y_{2}\right)$, so $E_{2}$ is path-connected.

We next show that $E$ is closed. Suppose that $\left(x_{r}, y_{r}\right) \in E$ and $\left(x_{r}, y_{r}\right) \rightarrow$ $(x, y)$. If $x=0$, then we note that, since $\left|y_{r}\right| \leq 1$ for all $r$ and $y_{r} \rightarrow y$, we have $|y| \leq 1$ and $(x, y) \in E_{1} \subseteq E$. If $x \neq 0$, then $1 \geq x>0$ (since $x_{r} \geq 0$ for all $r$ ). We can find an $N$ such that $\left|x-x_{r}\right|<x / 2$ and so $x_{r}>x / 2$ for all $r \geq N$. Thus, by continuity,

$$
\left(x_{r}, y_{r}\right)=\left(x_{r}, \sin 1 / x_{r}\right) \rightarrow(x, \sin 1 / x) \in E_{2} \subseteq E .
$$

Thus $E$ is closed.

Now suppose, if possible, that $E$ is disconnected. Then we can find $U$ and $V$ open such that

$$
U \cap E \neq \varnothing, V \cap E \neq \varnothing, U \cup V \supseteq E \text { and } U \cap V \cap E=\varnothing .
$$

Then

$$
U \cup V \supseteq E_{j} \text { and } U \cap V \cap E_{j}=\varnothing .
$$

and so, since $E_{j}$ is path-connected, so connected, we have $U \cap E_{j}=\varnothing$ or $V \cap E_{j}=\varnothing[j=1,2]$. Without loss of generality, assume $V \cap E_{1}=\varnothing$ so 
$U \supseteq E_{1}$. Since $(0,0) \in E_{1}$, we have $(0,0) \in U$. Since $U$ is open, we can find a $\delta>0$ such that $(x, y) \in U$ whenever $\|(x, y)\|_{2}<\delta$. If $n$ is large,

$$
\left((n \pi)^{-1}, 0\right) \in U \cap E_{2}=U \cap V \cap E,
$$

contradicting our initial assumptions. By reductio ad absurdum, $E$ is connected.

(iii) Write $\mathbf{x}(t)=(x(t), y(t))$. Since $\mathbf{x}$ is continuous, so is $x$. Since $x(0)=1$ and $x(1)=0$, the intermediate value theorem tells us that we can find $t_{1}$ with $0<t_{1}<1$ and $x\left(t_{1}\right)=\left(\frac{3}{2} \pi\right)^{-1}$. Applying the intermediate value theorem again, we can find $t_{2}$ with $0<t_{2}<t_{1}$ and $x\left(t_{2}\right)=\left(\frac{5}{2} \pi\right)^{-1}$. We continue inductively.

Since the $t_{j}$ form a decreasing sequence bounded below by 0 , we have $t_{j} \rightarrow T$ for some $T \in[0,1]$. Since $y$ is continuous

$$
(-1)^{j}=\sin \left(1 / x\left(t_{j}\right)\right)=y\left(t_{j}\right) \rightarrow y(T)
$$

which is absurd.

(iv) Part (iii) tells us that there is no path joining $(0,0)$ and $(1,0)$ in $E$, so $E$ is not path-connected.

[Return to page 31.]

Example 12.1. Give an example of metric space $(X, d)$ which is bounded (in the sense that there exists an $M$ with $d(x, y) \leq M$ for all $x, y \in X$ ) but for which there exist sequences with no convergent subsequence.

Solution. Consider the discrete metric on $\mathbb{Z}$. If $x_{n}=n$ and $x \in \mathbb{Z}$, then $d\left(x, x_{n}\right)=1$ for all $n$ with at most one exception. Thus the sequence $x_{n}$ can have no convergent subsequence.

[Return to page 32.]

Theorem 12.4. If the metric space $(X, d)$ is compact, it is sequentially compact.

Proof. Let $x_{n}$ be a sequence in $X$. If it has no convergent subsequence, then, for each $x \in X$ we can find a $\delta(x)>0$ and an $N(x)$ such that $x_{n} \notin B(x, \delta(x))$ for all $n \geq N(x)$. Since

$$
X=\bigcup_{x \in X}\{x\} \subseteq \bigcup_{x \in X} B(x, \delta(x)) \subseteq X,
$$

the $B(x, \delta(x))$ form an open cover and, by compactness, have a finite subcover. In other words, we can find an $M$ and $y_{j} \in X[1 \leq j \leq M]$ such that

$$
X=\bigcup_{j=1}^{M} B\left(y_{j}, \delta\left(y_{j}\right)\right) \text {. }
$$


Now set $N=\max _{1 \leq j \leq M} N\left(y_{j}\right)$. Since $N \geq N\left(y_{j}\right)$, we have $x_{N} \notin$ $B\left(y_{j}, \delta\left(y_{j}\right)\right)$ for all $1 \leq j \leq M$. Thus $x_{N} \notin \bigcup_{j=1}^{M} B\left(y_{j}, \delta\left(y_{j}\right)\right)=X$ which is absurd.

The result follows by reductio ad absurdum.

[Return to page 32.]

Lemma 12.5. Suppose that $(X, d)$ is a sequentially compact metric space and that the collection $U_{\alpha}$ with $\alpha \in A$ is an open cover of $X$. Then there exists a $\delta>0$ such that, given any $x \in X$, there exists an $\alpha(x) \in A$ such that the open ball $B(x, \delta) \subseteq U_{\alpha(x)}$.

Proof. Suppose the first sentence is true and the second sentence false. Then, for each $n \geq 1$ we can find an $x_{n}$ such that the open ball $B\left(x_{n}, 1 / n\right) \nsubseteq U_{\alpha}$ for all $\alpha \in A$. By sequential compactness, we can find $y \in X$ and $n(j) \rightarrow \infty$ such that $x_{n(j)} \rightarrow y$.

Since $y \in X$, we must have $y \in U_{\beta}$ for some $\beta \in A$. Since $U_{\beta}$ is open, we can find an $\epsilon$ such that $B(y, \epsilon) \subseteq U_{\beta}$. Now choose $J$ sufficiently large that $n(J)>2 \epsilon^{-1}$ and $d\left(x_{n(J)}, y\right)<\epsilon / 2$. We now have, using the triangle inequality, that

$$
B\left(x_{n(J)}, 1 / n(J)\right) \subseteq B\left(x_{n(J)}, \epsilon / 2\right) \subseteq B(y, \epsilon) \subseteq U_{\beta},
$$

contradicting the definition of $x_{n(J)}$.

The result follows by reductio ad absurdum.

[Return to page 32.]

Theorem 12.6. If the metric space $(X, d)$ is sequentially compact, it is compact.

Proof. Let $\left(U_{\alpha}\right)_{\alpha \in A}$ be an open cover and let $\delta$ be defined as in Lemma 12.5. The $B(x, \delta)$ form a cover of $X$. If they have no finite subcover, then given $x_{1}$, $x_{2}, \ldots x_{n}$ we can find an $x_{n+1} \notin \bigcup_{j=1}^{n} B\left(x_{j}, \delta\right)$. Consider the sequence $x_{j}$ thus obtained. We have $d\left(x_{n+1}, x_{k}\right)>\delta$ whenever $n \geq k \geq 1$ and so $d\left(x_{r}, x_{s}\right)>\delta$ for all $r \neq s$. It follows that, if $x \in X, d\left(x_{n}, x\right)>\delta / 2$ for all $n$ with at most one exception. Thus the sequence of $x_{n}$ has no convergent subsequence.

It thus follows, by reductio ad absurdum, that the $B(x, \delta)$ have a finite subcover. In other words, we can find an $M$ and $y_{j} \in X[1 \leq j \leq M]$ such that

$$
X=\bigcup_{j=1}^{M} B\left(y_{j}, \delta\right) .
$$


We thus have

$$
X=\bigcup_{j=1}^{M} B\left(y_{j}, \delta\right) \subseteq \bigcup_{j=1}^{M} U_{\alpha\left(y_{j}\right)} \subseteq X
$$

so $X=\bigcup_{j=1}^{M} U_{\alpha\left(y_{j}\right)}$ and we have found a finite subcover.

Thus $X$ is compact.

[Return to page 32.]

Lemma 13.2. Let $(X, \tau)$ be a topological space. Then $U \in \tau$ if and only if, given $x \in U$, we can find a neighbourhood $N$ of $x$ with $N \subseteq U$.

Proof. If $U \in \tau$ then $U$ is a neighbourhood of $x$ for all $x \in U$.

Conversely, if given any $x \in U$, we can find a neighbourhood $N_{x}$ of $x$ with $N_{x} \subseteq U$, then we can find an open neighbourhood $U_{x}$ of $x$ with $U_{x} \subseteq N_{x}$. Since

$$
U \subseteq \bigcup_{x \in U}\{x\} \subseteq \bigcup_{x \in U} U_{x} \subseteq \bigcup_{x \in U} N_{x} \subseteq \bigcup_{x \in U} U=U
$$

we have $U=\bigcup_{x \in U} U_{x} \in \tau$.

[Return to page 34.]

Lemma 13.3. Let $(X, \tau)$ and $(Y, \sigma)$ be topological spaces. Then $f: X \rightarrow Y$ is continuous if and only if, given $x \in X$ and $M$ a neighbourhood of $f(x)$ in $Y$, we can find a neighbourhood $N$ of $x$ with $f(N) \subseteq M$.

Proof. If If $f: X \rightarrow Y$ is continuous, $x \in X$ and $M$ is a neighbourhood of $f(x)$, then we can find a $V \in \sigma$ with $f(x) \in V \subseteq M$. Since $f$ is continuous $f^{-1}(V) \in \tau$. Thus, since $x \in f^{-1}(V)$, we have that $f^{-1}(V)$ is an open neighbourhood and so a neighbourhood of $x$. Setting $N=f^{-1}(V)$, we have $f(N)=V \subseteq M$ as required.

Only if Suppose that, given $x \in X$ and $M$ a neighbourhood of $f(x)$ in $Y$, we can find a neighbourhood $N$ of $x$ with $f(N) \subseteq M$. Let $V$ be open in $Y$. If $x \in X$ and $f(x) \in V$, then $V$ is a neighbourhood of $f(x)$ so there exists a neighbourhood $N_{x}$ of $x$ with $f\left(N_{x}\right) \subseteq V$. We now choose $U_{x}$ an open neighbourhood of $x$ with $U_{x} \subseteq N_{x}$. We have

$$
f\left(U_{x}\right) \subseteq V
$$

and so $U_{x} \subseteq f^{-1}(V)$ for all $x \in f^{-1}(V)$. Thus

$$
f^{-1}(V)=\bigcup_{x \in f^{-1}(V)}\{x\} \subseteq \bigcup_{x \in f^{-1}(V)} U_{x} \subseteq \bigcup_{x \in f^{-1}(V)} f^{-1}(V)=f^{-1}(V) .
$$


It follows that $f^{-1}(V)=\bigcup_{x \in f^{-1}(V)} U_{x} \in \tau$. We have shown that $f$ is continuous.

[Return to page 34.]

Lemma 13.6. Let $X$ be a set and $\mathcal{B}$ a collection of subsets of $X$. Let $\tau_{\mathcal{B}}$ be the collection of sets $U$ such that, whenever $x \in U$, we can find a $B \in \mathcal{B}$ such that $x \in B \subset U$.

Then $\tau_{\mathcal{B}}$ is a topology if and only if $\mathcal{B}$ is a basis.

Proof. We first prove necessity. If $\tau_{B}$ is a topology, then $X \in \tau_{\mathcal{B}}$ and so for each $x \in X$ we can find a $B_{x} \in \mathcal{B}$ with $x \in B_{x}$. Thus

$$
\bigcup_{B \in \mathcal{B}} B \supseteq \bigcup_{x \in X} B_{x} \supseteq \bigcup_{x \in X}\{x\}=X
$$

so $\bigcup_{B \in \mathcal{B}} B=X$.

Next we observe that, by definition, $\mathcal{B} \subseteq \tau_{\mathcal{B}}$. Thus if $B_{1}, B_{2} \in \mathcal{B}$ we must have $B_{1} \cup B_{2} \in \mathcal{B}$ and, by definition, if $x \in B_{1} \cup B_{2}$ we can find a $B_{3} \in \mathcal{B}$ such that $x \in B_{3} \subseteq B_{1} \cup B_{2}$. Thus $\mathcal{B}$ is a basis.

We now prove sufficiency. Suppose that $\mathcal{B}$ is a basis. We observe that, using the definition, $\mathcal{B} \subseteq \tau_{\mathcal{B}}$ and whenever $\mathcal{A} \subseteq \tau_{\mathcal{B}}$ we have $\bigcup_{A \in \mathcal{A}} A \in \tau_{\mathcal{B}}$.

We have $\varnothing \in \tau_{\mathcal{B}}$ vacuously and, by the definition of a basis, $X=$ $\bigcup_{B \in \mathcal{B}} B \in \tau_{\mathcal{B}}$.

Finally, if $U_{1}, U_{2} \in \tau_{B}$ then whenever $x \in U_{1} \cap U_{2}$ we can find $B_{1}, B_{2} \in \mathcal{B}$ with $x \in B_{1} \subset U_{1}, x \in B_{2} \subset U_{2}$. By the definition of a basis, we can find $B_{3} \in \mathcal{B}$ with

$$
x \in B_{3} \subseteq B_{1} \cap B_{2} \subseteq U_{1} \cap U_{2} .
$$

Thus $U_{1} \cap U_{2} \in \tau_{\mathcal{B}}$. Thus $\tau_{\mathcal{B}}$ is a topology.

[Return to page 34.]

\section{Executive summary}

\section{Metrics}

Definition and examples [Page 3]. Continuity [Page 7]. Open sets [Page 8]. Characterising continuous functions using open sets [Theorem 3.7, Page 9]. Limits [Page 10]. Closed sets [Page 11].

\section{Topology}

Definition of a topology [Page 12]. Metric topologies [Theorem 5.2, Page 12]. Further examples [Page 13]. Continuous functions [Page 13] and closed sets 
[Page 14]. Interior and closure [Page 14]. Dense subsets [Page 15] Homeomorphisms [Page 16]. Topological and non-topological properties [Page 16] illustrated by completeness [Page 16]. Subspace [Page 18], product [Page 18] and quotient [Lemma 7.15, Page 20] topologies. Hausdorff spaces [Page 20].

\section{Compactness}

Definition using open sets [Page 22]. Examples: finite sets [Example 9.4, Page 23] and [0,1] [Theorem 9.5, Page 23]. Closed subsets of compact sets are compact [Theorem 9.7, Page 24]. Compact subsets of a Hausdorff space must be closed [Theorem 9.8, Page 24]. The compact subsets of the real line [Theorem 9.10, Page 24]. Continuous images of compact sets are compact [Theorem 9.12, Page 24]. Quotient spaces and compactness [Page 25]. Continuous real valued functions on a compact space are bounded and attain their bounds [Theorem 9.17, Page 25]. The product of two compact spaces is compact [Theorem 10.1, Page 26]. The compact subsets of Euclidean space [Theorem 10.5, Page 27]. Sequential compactness [Page 32].

\section{Connectedness}

Definition using open sets [Page 28] and integer valued functions [Theorem 11.4, Page 29]. Examples, including intervals [Theorem 11.5, Page 29]. Continuous image of a connected set is connected [Example 11.6 (i), Page 29]. Components [Lemma 11.9, Page 30]. Path-connectedness [Page 30]. Pathconnected spaces are connected [Theorem 11.12, Page 31] but not conversely [Example 11.14, Page 31]. Connected open sets in Euclidean space are pathconnected [Theorem 11.13, Page 31].

\section{Neighbourhoods}

Open neighbourhoods [Page 21]. Neighbourhoods [Page 34]. Continuity via neighbourhoods [Lemma 13.3, Page 34]. Bases [Page 34]. Neighbourhoods [Exercise 13.4, Page 34] and convergence [Lemma 13.10, Page 35] in metric spaces. Limits treacherous concept in general topological spaces [Page 35]. 